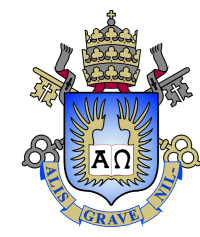

Fernando Silva Braga

\title{
Existência, Unicidade e Estabilidade de Soluções de Sistemas de Equações Diferenciais Ordinárias
}

Dissertação de Mestrado

Dissertação apresentada como requisito parcial para obtenção do grau de Mestre pelo Programa de Pós-graduação em Matemática, do Departamento de Matemática da PUC-Rio.

Orientador: Prof. Boyan Slavchev Sirakov 
Fernando Silva Braga

\section{Existência, Unicidade e Estabilidade de Soluções de Sistemas de Equações Diferenciais \\ Ordinárias}

Dissertação apresentada como requisito parcial para obtenção do grau de Mestre pelo Programa de Pós-graduação em Matemática da PUC-Rio. Aprovada pela Comissão Examinadora abaixo:

Prof. Boyan Slavchev Sirakov Orientador

Departamento de Matemática - PUC-Rio

Prof. Marcos Craizer

Departamento de Matemática - PUC-Rio

Prof. Carlos Hugo Jiménez Gómez

Departamento de Matemática - PUC-Rio

Prof. Edgard Almeida Pimentel Departamento de Matemática - PUC-Rio

Prof. Simon Griffiths

Departamento de Matemática - PUC-Rio 
Todos os direitos reservados. A reprodução, total ou parcial do trabalho, é proibida sem a autorização da universidade, do autor e do orientador.

\section{Fernando Silva Braga}

Graduado em Engenharia Civil na enfase de Métodos Numéricos do Departamento de Estruturas da Universidade Federal do Rio de Janeiro/UFRJ (2000), empresário de tecnologia da informação há 20 anos, com mais de 30 anos de experiência no desenvolvimento de softwares, tendo projetado, desenvolvido e implantado o primeiro sistema de emissão de notas fiscais eletrônicas do país.

Ficha Catalográfica

Silva Braga, Fernando

Existência, Unicidade e Estabilidade de Soluções de Sistemas de Equações Diferenciais Ordinárias / Fernando Silva Braga; orientador: Boyan Slavchev Sirakov. - 2021.

85 f: il. color. ; $30 \mathrm{~cm}$

Dissertação (mestrado) - Pontifícia Universidade Católica do Rio de Janeiro, Departamento de Matemática, 2021.

Inclui bibliografia

1. Matemática - Teses. 2. Análise e Equações Diferenciais - Teses. 3. EDO. 4. Equações Diferenciais Ordinárias. 5. Existência e Unicidade de Soluções. 6. Sistemas Gerais Parametrizados de EDO. 7. Sistemas Lineares de EDO. 8. Estabilidade de Soluções. I. Sirakov, Boyan. II. Pontifícia Universidade Católica do Rio de Janeiro. Departamento de Matemática. III. Título. 
Aos meus professores do Ensino Médio, in memoriam, Eduardo e Cláudio. Às minhas filhas Marina e Juliana, fontes de inspiração, motivação e orgulho. 


\section{Agradecimentos}

Aos meus pais, meus professores da vida, que sempre me apoiaram e ensinaram a valorizar a educação e os estudos.

Ao Eduardo Barbosa e Cláudio Veloso, meus professores de Matemática e Física no ensino médio pelos inestimáveis ensinamentos e incentivos.

Aos meus professores da Engenharia Civil da UFRJ Nelson Galgoul, Silvio Lima e Gilberto Ellwanger por elaborarem as cartas de recomendações necessárias para minha inscrição neste Mestrado.

Ao professor Marcos Craizer que me recebeu na PUC-RIO com toda simpatia e franqueza para uma conversa que foi decisiva para eu me inscrever no mestrado, me motivando e fornecendo valiosos conselhos desde então.

Ao meu orientador e professor Boyan Sirakov, não só por ter aceito o desafio de me orientar, mas principalmente por todo apoio e estímulo que recebi sempre que precisei. Tive a oportunidade de ser seu aluno no curso de Análise Real cuja abordagem tem a preocupação em aplicar os resultados em problemas de cálculo avançado. Fui também seu aluno no excelente curso de Equações Diferenciais Ordinárias, cujas notas de aulas e ensinamentos foram fundamentais para elaboração desta dissertação.

Ao professor Carlos Hugo Jimenez pela sua paciência e didática no curso de Introdução em Análise, que foram fundamentais para eu começar a ganhar ritmo, após tanto tempo em que estive afastado dos estudos.

À professora Alessia Mandini não só por suas aulas de Álgebra Linear, mas por me lembrar de uma importante lição: "Não se aprende matemática sem fazer exercícios. Faça o máximo que puder!". Assim foi feito.

Ao professor Alessandro Alla que me mostrou nas disciplinas computacionais uma abordagem muito interessante da matemática aplicada, ampliando meus conhecimentos em Álgebra Linear.

Ao professor Edgard Pimentel por sua empolgação contagiante e pelos conhecimentos que recebi no curso de Análise em $\mathbb{R}^{n}$

À secretária Creuza, sempre muito solícita e disposta a ajudar.

Ao meu sócio Gustavo e a todos meus colegas de trabalho que compreenderam meu afastamento do dia-a-dia na empresa.

À minha esposa e filhas por todo apoio e compreensão, entendendo que, em alguns momentos, não pude dar a merecida atenção.

À PUC-RIO pela isenção das taxas escolares.

À CAPES pelo auxílio concedido.

O presente trabalho foi realizado com apoio da Coordenação de Aperfeiçoamento de Pessoal de Nível Superior - Brasil (CAPES) - Código de Financiamento 001. 


\section{Resumo}

Silva Braga, Fernando; Sirakov, Boyan. Existência, Unicidade e Estabilidade de Soluções de Sistemas de Equações Diferenciais Ordinárias. Rio de Janeiro, 2021. 85p. Dissertação de Mestrado - Departamento de Matemática, Pontifícia Universidade Católica do Rio de Janeiro.

Esta dissertação tem o objetivo de aplicar os conceitos e ferramentas da Análise Real e Álgebra Linear num estudo sobre a teoria de existência, unicidade e estabilidade de soluções de sistemas de equações diferenciais ordinárias, considerando sistemas gerais parametrizados, lineares e não-lineares.

\section{Palavras-chave}

EDO; Equações Diferenciais Ordinárias; Existência e Unicidade de Soluções; Sistemas Gerais Parametrizados de EDO; Sistemas Lineares de EDO; Estabilidade de Soluções. 


\section{Abstract}

Silva Braga, Fernando; Sirakov, Boyan (Advisor). Existence, Uniquiness and Stability of Solutions of Ordinary Differential Equations Systems. Rio de Janeiro, 2021. 85p. Dissertação de Mestrado - Departamento de Matemática, Pontifícia Universidade Católica do Rio de Janeiro.

This dissertation aims to apply the concepts and tools of Real Analysis and Linear Algebra to the theory of existence, uniquiness and stability of solutions of ordinary differential equations systems, considering general parametric, linear and non-linear systems.

\section{Keywords}

ODE; Ordinary Differential Equations; Existence and Uniqueness of Solutions; Parametric General Systems of ODE; Linear Systems of ODE; Stability of Solutions. 


\section{Sumário}

$\begin{array}{llr}1 & \text { Introdução } & 9\end{array}$

2 Sistemas Gerais Parametrizados de EDO 10

2.1 Existência e Unicidade da Solução 11

$\begin{array}{lll}2.2 & \text { Domínio Maximal de Existência da Solução } & 17\end{array}$

$\begin{array}{lll}2.3 & \text { Diferenciabilidade da Solução com respeito aos parâmetros } & 18\end{array}$

2.4 Propriedade de Saída do Compacto 20

$3 \quad$ Sistemas Lineares de EDO $\quad 23$

3.1 Domínio de Existência e Unicidade da Solução 23

3.2 A Matriz Fundamental e suas propriedades 26

3.3 Solução de Sistemas Lineares com coeficientes variáveis 33

3.4 A Exponencial Matricial 34

3.5 Solução de Sistemas Lineares com Matriz constante 42

4 Estabilidade de Soluções de Sistemas de EDO 44

4.1 Estabilidade Assintótica de Soluções 46

4.2 Instabilidade de Soluções $\quad 51$

4.3 Estabilidade em Sistemas Autônomos 55

5 Considerações Finais $\quad 57$

$\begin{array}{ll}\text { Referências bibliográficas } & 58\end{array}$

A Apêndice - Demonstração de Resultados Utilizados $\quad 59$

B Apêndice - Análise Real e Álgebra Linear 68

B.1 Espaço Vetorial 68

$\begin{array}{lll}\text { B.2 Normas } & 70\end{array}$

$\begin{array}{lll}\text { B.3 Topologia } & 72\end{array}$

B.4 Sequências e Séries $\quad 73$

$\begin{array}{lll}\text { B.5 Limites e Continuidade } & 75 \\ \text { B.6 }\end{array}$

$\begin{array}{lll}\text { B.6 Diferenciabilidade e Integração } & 77\end{array}$

B.7 Convergência Uniforme $\quad 80$

B.8 Matrizes $\quad 82$

B.9 Aplicações Lineares $\quad 84$

B.10 Autovalores e a Forma Normal de Jordan 85 


\section{1 \\ Introdução}

Esta dissertação tem o objetivo de aprofundar os conhecimentos de Análise Real aplicando os conceitos e ferramentas da Álgebra Linear num estudo com demonstrações detalhadas de assuntos relacionados a teoria geral dos sistemas de equações diferenciais ordinárias - EDO.

No Capítulo 2 é apresentada a teoria geral dos sistemas de EDO parametrizados $x^{\prime}=f(t, x, u)\left(u \in \mathbb{R}^{m}\right.$ parâmetro) contemplando um estudo sobre a existência e unicidade da solução, a análise de sua continuidade e diferenciabilidade com respeito aos parâmetros, seu domínio maximal de existência e a propriedade de saída do compacto.

No Capítulo 3 tratamos dos sistemas lineares com coeficientes variáveis $x^{\prime}=A(t) x+b(t)$ e o caso particular em que a matriz $A(t)=A \in \mathbb{R}^{n \times n}$ é uma constante. Neste capítulo, analisamos o domínio de existência e unicidade da solução, definimos a matriz fundamental de soluções e a exponencial matricial, demonstrando suas principais propriedades e aplicando os resultados para encontrar a solução destes sistemas.

No Capítulo 4, é analisada a estabilidade das soluções de sistemas lineares e não-lineares $\left(x^{\prime}=A x+f(t, x)\right)$ contemplando as condições para estabilidade assintótica e instabilidade das soluções.

Após as considerações finais são incluídas, como apêndices, as principais definições, teoremas e demonstrações de resultados provenientes da Análise Real e Álgebra Linear cujo conhecimento colabora para um melhor entendimento deste estudo. 


\section{Sistemas Gerais Parametrizados de EDO}

Considere os conjuntos abertos e conexos $[2] I \subseteq \mathbb{R}, X \subseteq \mathbb{R}^{n}$ e $U \subseteq \mathbb{R}^{m}$ em que $n$ e $m \in \mathbb{N}$. Seja $\Gamma$ um conjunto de funções contínuas definidas em $I \times X \rightarrow \mathbb{R}^{n}$. Fixe $t_{0} \in I$ e suponha que para todo $u \in U$ existem $g_{u} \in \mathbb{R}^{n} \mathrm{e}$ $f_{u} \in \Gamma$ tais que podemos definir o sistema de EDO:

$$
\left\{\begin{array}{l}
x_{u}^{\prime}(t)=f_{u}\left(t, x_{u}(t)\right) \\
x_{u}\left(t_{0}\right)=g_{u}
\end{array} .\right.
$$

Sejam $G:=I \times X \times U, f: G \rightarrow \mathbb{R}^{n}$ e $g: U \rightarrow \mathbb{R}^{n}$ tais que para todo $u \in U$ temos

$$
f(t, x(t, u), u):=f_{u}\left(t, x_{u}(t)\right) \quad \text { e } \quad g(u):=g_{u} .
$$

Suponha $f$ e $g$ contínuas e considere agora o seguinte sistema parametrizado por $u$ :

$$
\left\{\begin{array}{l}
\frac{\partial x}{\partial t}(t, u)=f(t, x(t, u), u) \\
x\left(t_{0}, u\right)=g(u)
\end{array}\right.
$$

Resolver o sistema (2-3) é equivalente a buscar, para todo $u \in U$, uma solução $x(t, u)=x_{u}(t)$ definida para algum intervalo $I_{u} \subseteq I$ em que $x_{u}(t)$ é solução do sistema (2-1) neste intervalo.

Neste capítulo, consideraremos fixos $u_{0} \in U$ e $x_{0}=g\left(u_{0}\right)$, suporemos $f$ localmente Lipschitz com respeito a $x$ em $G$ (Definição B.29) e buscaremos um compacto $U_{0} \subset U$ em que $u_{0} \in U_{0}$ e para todo $u \in U_{0}$ existe única solução $x$ para este sistema definida para algum intervalo fechado $I_{0} \subset I$.

Veremos que a função $x(t, u)$ é contínua no compacto $\Omega:=I_{0} \times U_{0} \mathrm{e}$ que, adicionando as hipóteses de que $f \in C^{1}(G)$ e $g$ é diferenciável em $U$, temos também que a função $x(t, u) \in C^{1}(\Omega)$. Definiremos também o intervalo maximal de existência de uma solução e veremos a propriedade de saída de um compacto. 


\section{1}

\section{Existência e Unicidade da Solução}

Nesta seção, demonstraremos um teorema fundamental das equações diferenciais ordinárias que estabelece condições para garantir a existência e unicidade de soluções para o sistema (2-3).

Inicialmente, notemos que, seja um conjunto compacto $\Pi \subset G$ então, pela Proposição A.1, como $f$ é localmente Lipschitz com respeito a $x$ em $G$ temos que $f$ é Lipschitz com respeito a $x$ em $\Pi$. Além disto, como $\Pi$ é um compacto e $f$ é contínua, pelo Teorema B.28, podemos definir $M:=\max _{\Pi}\|f(t, x, u)\|$.

Observemos também que, seja $b>0$ então pela continuidade de $g$ em $u_{0}$ existe $c>0$ tal que se $\left\|u-u_{0}\right\| \leq c$ temos $\left\|g(u)-g\left(u_{0}\right)\right\|=\left\|g(u)-x_{0}\right\|<\frac{b}{2}$.

Teorema 2.1 Considere o sistema (2-3) e suponha $f$ localmente Lipschitz com respeito a $x$ em $G$. Seja $\Pi \subset G$ definido como

$$
\Pi:=\left\{(t, x, u):\left|t-t_{0}\right| \leq a,\left\|x-x_{0}\right\| \leq b,\left\|u-u_{0}\right\| \leq c\right\}
$$

com $a, b, c>0$ tais que se $\left\|u-u_{0}\right\| \leq$ c temos $\left\|g(u)-x_{0}\right\|<\frac{b}{2}$.

Considere $L$ a constante de Lipschitz de $f$ com respeito a $x$ em $\Pi$ e sejam

$$
M:=\max _{\Pi}\|f(t, x, u)\| \text { e } h:=\min \left\{a, \frac{b}{2 M}, \frac{1}{2 L}\right\} .
$$

Então, para todo $u \in U$ tal que $\left\|u-u_{0}\right\| \leq c$, existe uma única solução $x$ deste sistema definida para todo $t \in\left[t_{0}-h, t_{0}+h\right]$. Mais ainda, a função $x(t, u)$ é contínua em $\Omega$ definido como

$$
\Omega:=I_{0} \times U_{0}:=\left\{(t, u):\left|t-t_{0}\right| \leq h,\left\|u-u_{0}\right\| \leq c\right\} .
$$

Demonstração.

Considere a sequência de funções $\left\{x_{k}\right\}_{k \in \mathbb{N}}$ em que definimos $x_{k}: \Omega \rightarrow \mathbb{R}^{n}$ para todo $k \in \mathbb{N}$ conforme a seguir:

$$
\begin{aligned}
& x_{0}(t, u):= x_{0} \\
& x_{1}(t, u):=g(u)+\int_{t_{0}}^{t} f\left(s, x_{0}(s, u), u\right) d s \\
& x_{2}(t, u):=g(u)+\int_{t_{0}}^{t} f\left(s, x_{1}(s, u), u\right) d s \\
& \\
& \quad \ldots \ldots \\
& x_{k}(t, u):=g(u)+\int_{t_{0}}^{t} f\left(s, x_{k-1}(s, u), u\right) d s .
\end{aligned}
$$

Mostraremos que $x_{k}$ está bem definida sendo contínua para todo $k \in \mathbb{N}$, bem como $x_{k}$ converge uniformemente para uma função $x$ contínua em $\Omega$ e veremos que, para todo $u \in U_{0}, x(t, u)$ é a única solução definida para todo $t \in I_{0}$. 
Afirmação (I): $x_{k}$ está bem definida e é contínua em $\Omega$ para todo $k \in \mathbb{N}$. Seja $(t, u) \in \Omega$ então $t \in\left[t_{0}-h, t_{0}+h\right], \operatorname{logo}\left|t-t_{0}\right| \leq a ;\left\|u-u_{0}\right\| \leq c$. Assim, mostraremos por indução em $k$ que $x_{k}$ é contínua e $\left\|x_{k}(t, u)-x_{0}\right\| \leq b$, ou seja, que $\left(t, x_{k}(t, u), u\right) \in \Pi$ e portanto $\left(t, x_{k}(t, u), u\right)$ pertence ao domínio de definição da função $f$ e logo a função $x_{k}$ está bem definida para todo $k \in \mathbb{N}$. Base da Indução: Se $k=1$ temos

$$
\begin{aligned}
\left\|x_{1}(t, u)-x_{0}\right\| & =\left\|g(u)+\int_{t_{0}}^{t} f\left(s, x_{0}, u\right) d s-x_{0}\right\| \\
& \leq\left\|g(u)-x_{0}\right\|+\left|\int_{t_{0}}^{t}\left\|f\left(s, x_{0}, u\right)\right\| d s\right| \leq \frac{b}{2}+\left|\int_{t_{0}}^{t} M d s\right| \\
& \leq \frac{b}{2}+M\left|t-t_{0}\right| \leq \frac{b}{2}+M h \leq \frac{b}{2}+M \frac{b}{2 M}=b .
\end{aligned}
$$

Como $g$ e $f$ são contínuas então, pelo Teorema Fundamental do Cálculo B.40, $x_{1}$ é a soma de funções contínuas e, pelo Teorema B.30, $x_{1}$ é contínua em $\Omega$. Passo Indutivo: Suponha válido para $(k-1)$ que $\left\|x_{k-1}(t, u)-x_{0}\right\| \leq b$ e que $x_{k-1}$ é contínua. Com isto, temos

$$
\begin{aligned}
\left\|x_{k}(t, u)-x_{0}\right\| & \leq\left\|g(u)-x_{0}\right\|+\left|\int_{t_{0}}^{t}\left\|f\left(s, x_{k-1}(s, u), u\right)\right\| d s\right| \\
& \leq \frac{b}{2}+M\left|t-t_{0}\right| \leq \frac{b}{2}+M \frac{b}{2 M}=b .
\end{aligned}
$$

Assim, $\left(t, x_{k}(t, u), u\right) \in \Pi \subset G$ e portanto $x_{k}$ está bem definida. Como $x_{k-1} \mathrm{e}$ $f$ são contínuas então, pelo Teorema B.30, a função composta $f\left(t, x_{k}(t, u), u\right)$ é contínua e logo, pelo Teorema Fundamental do Cálculo B.40, sua integral existe e é contínua. Como $g$ é contínua então $x_{k}$ é a soma de funções contínuas e portanto $x_{k}$ é contínua para todo $k \in \mathbb{N}$.

Afirmação (II): $x_{k}$ converge uniformemente em $\Omega$ para uma função $x$ que é contínua em $\Omega$.

Mostraremos que existe uma série convergente $\sum a_{k}$ tal que para todo $k \in \mathbb{N}$ tem-se $\left\|x_{k+1}(t, u)-x_{k}(t, u)\right\| \leq a_{k}$.

Vimos em (2-5) que $\left\|x_{1}(t, u)-x_{0}\right\| \leq b=: a_{1}$. Analogamente, temos:

$$
\begin{aligned}
\left\|x_{2}(t, u)-x_{1}(t, u)\right\| & =\| \int_{t_{0}}^{t}\left(f\left(s, x_{1}(s, u), u\right)-f\left(s, x_{0}, u\right)\right) d s \mid \\
& \leq\left|\int_{t_{0}}^{t}\left\|f\left(s, x_{1}(s, u), u\right)-f\left(s, x_{0}, u\right)\right\| d s\right| \\
& \leq\left|\int_{t_{0}}^{t} L\left\|x_{1}(s, u)-x_{0}\right\| d s\right| \\
& \leq L\left\|x_{1}(t, u)-x_{0}\right\|\left|t-t_{0}\right| \\
& \leq L b\left|t-t_{0}\right|=: a_{2} .
\end{aligned}
$$




$$
\begin{aligned}
\left\|x_{3}(t, u)-x_{2}(t, u)\right\| & =\left\|\int_{t_{0}}^{t}\left(f\left(s, x_{2}(s, u), u\right)-f\left(s, x_{1}(s, u), u\right)\right) d s\right\| \\
& \leq\left|\int_{t_{0}}^{t} L\left\|x_{2}(s, u)-x_{1}(s, u)\right\| d s\right| \\
& \leq\left|\int_{t_{0}}^{t} L^{2} b\right| s-t_{0}|d s| \leq L^{2} b \frac{\left|t-t_{0}\right|^{2}}{2}=: a_{3} . \\
\left\|x_{4}(t, u)-x_{3}(t, u)\right\| & \leq\left|\int_{t_{0}}^{t} L\left\|x_{3}(s, u)-x_{2}(s, u)\right\| d s\right| \\
& \leq\left|\int_{t_{0}}^{t} \frac{L^{3} b}{2}\right| s-\left.t_{0}\right|^{2} d s \mid \leq L^{3} b \frac{\left|t-t_{0}\right|^{3}}{3.2}=: a_{4} .
\end{aligned}
$$

Procedendo assim para todo $k \in \mathbb{N}$ temos

$$
\left\|x_{k+1}(t, u)-x_{k}(t, u)\right\| \leq \frac{L^{k} b}{k !}\left|t-t_{0}\right|^{k} \leq b \frac{(L h)^{k}}{k !}=: a_{k} .
$$

Seja a sequência de funções $z_{k}$ tal que para todo $k \in \mathbb{N}$ temos

$$
z_{k}:=x_{k}-x_{0}=x_{k}-x_{k-1}+x_{k-1}-x_{k-2}+x_{k-2}+\cdots-x_{1}+x_{1}-x_{0} .
$$

Logo

$$
z_{k}=\sum_{i=1}^{k}\left(x_{i}-x_{i-1}\right)
$$

Mas, pela Definição B.21, $\sum_{k=1}^{\infty} a_{k}$ converge para $b\left(e^{L h}-1\right)$ e logo, pelo Teorema de Weierstrass A.4, $z_{k}$ converge uniformemente em $\Omega$ e portanto $x_{k}$ converge uniformemente em $\Omega$. Assim podemos definir para todo $(t, u) \in \Omega$

$$
x(t, u):=\lim _{k \rightarrow \infty} x_{k}(t, u) .
$$

Como $x_{k}$ é contínua em $\Omega$ para todo $k \in \mathbb{N}$ então, pelo Teorema B.45, $x$ é contínua em $\Omega$.

Afirmação (III): $\lim _{k \rightarrow \infty} \int_{t_{0}}^{t} f\left(s, x_{k}(s, u), u\right) d s=\int_{t_{0}}^{t} f(s, x(s, u), u) d s$.

Como $f$ é Lipschitz com respeito a $x$ em $\Pi$, pela Definição B.29

$$
\begin{aligned}
\left\|\int_{t_{0}}^{t}\left(f\left(s, x_{k}(s, u), u\right)-f(s, x(s, u), u)\right) d s\right\| & \leq\left|\int_{t_{0}}^{t} L\left\|x_{k}(s, u)-x(s, u)\right\| d s\right| \\
& \leq\left|\int_{t_{0}}^{t} L \sup _{(s, u) \in \Omega}\left\|x_{k}(s, u)-x(s, u)\right\| d s\right| \\
& \leq L \sup _{(s, u) \in \Omega}\left\|x_{k}(s, u)-x(s, u)\right\|\left|t-t_{0}\right| \\
& \leq L h \sup _{(s, u) \in \Omega}\left\|x_{k}(s, u)-x(s, u)\right\| .
\end{aligned}
$$


Já que $x_{k}$ converge uniformemente para $x$ em $\Omega$, pela Definição B.27, quando $k \rightarrow \infty$ temos

$$
\sup _{(s, u) \in \Omega}\left\|x_{k}(s, u)-x(s, u)\right\| \rightarrow 0
$$

e $\log 0$

$$
\int_{t_{0}}^{t} f\left(s, x_{k}(s, u), u\right) d s \rightarrow \int_{t_{0}}^{t} f(s, x(s, u), u) d s
$$

Note que, pode-se perceber mais diretamente esta convergência pois, conforme Proposição A.6, quando $k \rightarrow \infty$ temos que $f\left(t, x_{k}(t, u), u\right) \rightarrow f(t, x(t, u), u)$ uniformemente com respeito a $t$ em $\Omega$, mesmo nos casos em que $f$ é apenas contínua (não necessariamente Lipschitz). Com isto, podemos usar diretamente o resultado do Teorema B.49 e obter $\int_{t_{0}}^{t} f\left(s, x_{k}(s, u), u\right) d s \rightarrow \int_{t_{0}}^{t} f(s, x(s, u), u) d s$.

Afirmação (IV): Para todo $u \in U_{0}$ temos que $x(t, u)$ é solução de (2-3) definida para todo $t \in I_{0}$.

Fixemos $u \in U_{0}$ arbitrário. De (2-4)

$$
x_{k}(t, u)=g(u)+\int_{t_{0}}^{t} f\left(s, x_{k-1}(s, u), u\right) d s \quad \forall t \in I_{0} .
$$

De (II) e (III), quando $k \rightarrow \infty$ temos

$$
x(t, u)=g(u)+\int_{t_{0}}^{t} f(s, x(s, u), u) d s \quad \forall t \in I_{0} .
$$

Assim, $x\left(t_{0}, u\right)=g(u)$ e portanto

$$
x(t, u)=x\left(t_{0}, u\right)+\int_{t_{0}}^{t} f(s, x(s, u), u) d s \quad \forall t \in I_{0} .
$$

Já que $x$ e $f$ são contínuas então, pelo Teorema B.30, sua composta $f(t, x(t, u), u)$ também é contínua e, pelo Teorema Fundamental do Cálculo B.40, $x(t, u)$ é tal que

$$
\frac{\partial x}{\partial t}(t, u)=f(t, x(t, u), u)
$$

Como $x\left(t_{0}, u\right)=g(u)$ e $u$ é arbitrário então para todo $u \in U_{0}$ temos que $x(t, u)$ é solução de (2-3) definida para todo $t \in I_{0}$. 
Afirmação (V): Para todo $u \in U_{0}$ temos que $x(t, u)$ é a única solução de (2-3) definida para todo $t \in I_{0}$.

Fixemos $u \in U_{0}$ arbitrário. De (IV), temos que $x(t, u)$ é solução definida para todo $t \in I_{0}$.

Suponha por absurdo existir outra solução $y$ definida para todo $t \in\left[t_{0}-\delta, t_{0}+\delta\right]$ para algum $\delta>0$. Sejam $h_{1}=\min \{\delta, h\}$ e $I_{1}:=\left[t_{0}-h_{1}, t_{0}+h_{1}\right]$.

Como $I_{1} \subseteq I_{0}$ então $x$ e $y$ são soluções para todo $t \in I_{1}$, logo

$$
\begin{aligned}
& x(t, u)=g(u)+\int_{t_{0}}^{t} f(s, x(s, u), u) d s \\
& y(t, u)=g(u)+\int_{t_{0}}^{t} f(s, y(s, u), u) d s .
\end{aligned}
$$

Como $x$ e $y$ são contínuas em um compacto então a função $(x-y)$ também é contínua num compacto e, pelo Teorema B.28, existe $t_{1} \in I_{1}$ tal que

Como $f$ é Lipschitz com respeito a $x$ em $\Pi$

$$
\begin{aligned}
\|x(t, u)-y(t, u)\| & \leq\left\|\int_{t_{0}}^{t}(f(s, x(s, u), u)-f(s, y(s, u), u)) d s\right\| \\
& \leq\left|\int_{t_{0}}^{t} L\|x(s, u)-y(s, u)\| d s\right| \\
& \leq L\left|\int_{t_{0}}^{t} M_{1} d s\right| \leq L M_{1}\left|t-t_{0}\right| \leq L M_{1} h_{1} .
\end{aligned}
$$

Assim $\|x(t, u)-y(t, u)\| \leq L M_{1} h_{1}$ para todo $t \in I_{1}$ e como $t_{1} \in I_{1}$ temos

$$
M_{1}=\left\|x\left(t_{1}, u\right)-y\left(t_{1}, u\right)\right\| \leq L M_{1} h_{1} \Rightarrow h_{1} \geq \frac{1}{L} .
$$

Absurdo, já que $h_{1}=\min \{\delta, h\} \leq h \leq \frac{1}{2 L}<\frac{1}{L}$.

Como $u$ é arbitrário, para todo $u \in U_{0}$ existe única solução $x$ do sistema (2-3) definida para todo $t \in I_{0}$.

A seguir, mostraremos que se existirem duas soluções de (2-3) definidas em intervalos abertos distintos então estas duas soluções são iguais na interseção destes intervalos. 
Teorema 2.2 Considere o sistema (2-3) e fixe $u \in U$. Suponha $f$ localmente Lipschitz com respeito a $x$ em $G$. Se existirem duas soluçôes $x_{1}$ e $x_{2}$ definidas, respectivamente, em intervalos abertos $I_{1}$ e $I_{2}$ então $x_{1}(t, u)=x_{2}(t, u)$ para todo $t \in I_{1} \cap I_{2}$.

Demonstração.

Seja $(c, d):=I_{1} \cap I_{2}$. Como $x_{1}$ e $x_{2}$ são soluções em $(c, d)$ então $t_{0} \in(c, d)$. Suponha, inicialmente, $d \in \mathbb{R}$ (finito).

Sejam:

$$
P:=\left\{t \in\left[t_{0}, d\right): x_{1}\left(t^{\prime}, u\right)=x_{2}\left(t^{\prime}, u\right) \forall t^{\prime} \in\left[t_{0}, t\right)\right\} \text { e } T:=\sup P .
$$

Pelo Teorema 2.1 (considerando $u_{0}=u$ ), existe $h>0$ tal que $x_{1}(t, u)=x_{2}(t, u)$ para todo $t \in\left(t_{0}-h, t_{0}+h\right)$ e $\log o T>t_{0}$.

Afirmação (I): $x_{1}(t, u)=x_{2}(t, u)$ para todo $t \in\left[t_{0}, T\right)$.

Seja $t \in\left[t_{0}, T\right)$ arbitrário. Pela definição de supremo, existe $t_{1} \in P$ tal que $t<t_{1}<T$. Como $t_{1} \in P$ então $x_{1}\left(t^{\prime}, u\right)=x_{2}\left(t^{\prime}, u\right)$ para todo $t^{\prime} \in\left[t_{0}, t_{1}\right)$.

Escolhendo $t^{\prime}=t$ temos $x_{1}(t, u)=x_{2}(t, u)$. Como $t$ é arbitrário então $x_{1}(t, u)=x_{2}(t, u)$ para todo $t \in\left[t_{0}, T\right)$.

Afirmação (II): $T=d$.

Suponha por absurdo que $T<d$. Seja a sequência $\left(t_{n}\right)_{n \in \mathbb{N}}$ tal que $t_{n}=T-\frac{1}{n}$ para todo $n \in \mathbb{N}$. Como $t_{n} \rightarrow T$ então existe $N \in \mathbb{N}$ tal que $t_{n} \in\left(t_{0}, T\right)$ para todo $n>N$. Assim, por (I) temos que $x_{1}\left(t_{n}, u\right)=x_{2}\left(t_{n}, u\right)$ para todo $n>N$. Logo, quando $n \rightarrow \infty$ temos $x_{1}(T, u)=x_{2}(T, u)$.

Considere o sistema:

$$
\left\{\begin{array}{l}
\frac{\partial x}{\partial t}(t, u)=f(t, x(t, u), u) \\
x(T, u)=x_{1}(T, u)
\end{array} .\right.
$$

Pelo Teorema de Existência e Unicidade 2.1 (considerando $\left(t_{0}, u_{0}\right)=(T, u)$ ), existe uma única solução para este sistema em $(T-H, T+H)$ para algum $H>0$. Escolhemos $\delta<H$ suficientemente pequeno tal que $(T-\delta, T+\delta) \subset(c, d)$. Como $x_{1}$ e $x_{2}$ são soluções deste sistema em $(T-\delta, T+\delta)$ então $x_{1}(t, u)=x_{2}(t, u)$ para todo $t \in\left(t_{0}, T+\delta\right)$. Absurdo, pois $T=\sup P \geq T+\delta$. Logo $T=d$.

Se $d$ não for finito, consideramos a sequência de intervalos $\alpha_{n}:=\left(t_{0}, n\right)$ definida para todo $n \in \mathbb{N}$ e usamos o resultado anterior para cada um dos intervalos limitados $\alpha_{n}$ e temos que $x_{1}(t, u)=x_{2}(t, u)$ em $\left(t_{0}, n\right)$ para todo $n \in \mathbb{N}, \operatorname{logo} x_{1}(t, u)=x_{2}(t, u)$ para todo $t \in\left(t_{0}, \infty\right)$. 
Por fim, procedemos analogamente para $c$, porém definindo:

$$
\left.P_{1}:=\left\{t \in\left(c, t_{0}\right]: x_{1}\left(t^{\prime}, u\right)=x_{2}\left(t^{\prime}, u\right) \forall t^{\prime} \in\left(t, t_{0}\right]\right)\right\} \text { e } T_{1}:=\inf P_{1}
$$

e concluímos que $T_{1}=c$ obtendo o resultado procurado.

\section{2}

\section{Domínio Maximal de Existência da Solução}

Considerando agora o sistema (2-3), mostraremos que se $f$ for localmente Lipschitz com respeito a $x$ em $G$ então existe um intervalo $I$ além do qual a solução $\varphi$ deste sistema não pode ser prolongada. Denominamos $\varphi$ solução não-prolongável do sistema e $I$ intervalo (ou domínio) maximal de existência da solução.

Teorema 2.3 Considere o sistema (2-3) e fixe $u \in U$. Se $f$ é localmente Lipschitz com respeito a $x$ em $G$ então existe um intervalo de comprimento maximal $\left(m_{1}, m_{2}\right) \subseteq \mathbb{R}$ no qual é definida uma solução $\varphi$ não-prolongável $e$ única.

\section{Demonstração.}

Pelo Teorema 2.1, existe única solução do sistema em um intervalo $\left(t_{0}-h, t_{0}+h\right)$ para algum $h>0$. Assim, podemos definir:

$m_{1}:=\inf \left\{\alpha<t_{0}\right.$ tal que existe solução do sistema no intervalo $\left.\left(\alpha, t_{0}+h\right)\right\}$ $m_{2}:=\sup \left\{\beta>t_{0}\right.$ tal que existe solução do sistema no intervalo $\left.\left(t_{0}-h, \beta\right)\right\}$ em que $m_{1}$ e/ou $m_{2}$ podem ou não ser finitos.

Seja $t \in\left(m_{1}, m_{2}\right)$ fixo e arbitrário. Como $t>m_{1}$, pela definição de ínfimo, existe $\alpha<t$ tal que existe solução $\psi_{1}$ em algum intervalo $\left(\alpha, t_{0}+h\right)$ que contém $t$. Analogamente, como $t<m_{2}$, pela definição de supremo, existe $\beta>t$ tal que existe solução $\psi_{2}$ em algum intervalo $\left(t_{0}-h, \beta\right)$ que contém $t$.

Assim, definimos uma função $\varphi_{1}$ para todo $t \in I_{1}:=(\alpha, \beta) \subseteq\left(m_{1}, m_{2}\right)$ tal que

$$
\varphi_{1}(t, u):=\left\{\begin{array}{ll}
\psi_{1}(t, u) & \text { se } t \in\left(\alpha, t_{0}\right] \\
\psi_{2}(t, u) & \text { se } t \in\left(t_{0}, \beta\right)
\end{array} .\right.
$$

Pelo Teorema 2.1, $\psi_{1}(t, u)=\psi_{2}(t, u)$ para todo $t \in\left(t_{0}-h, t_{0}+h\right)$ e $\operatorname{logo} \varphi_{1}$ é uma função bem definida e contínua, sendo uma solução para todo $t \in(\alpha, \beta)$. Seja $\varphi_{2}$ uma outra solução definida num intervalo $I_{2}$ qualquer que contenha $t$ então, pelo Teorema $2.2, \varphi_{1}(t, u)=\varphi_{2}(t, u)$ para todo $t \in I_{1} \cap I_{2}$. 
Como $t$ é arbitrário, podemos definir $\varphi(t, u):=\varphi_{1}(t, u)$ para todo $t \in\left(m_{1}, m_{2}\right)$. Derivando em t

$$
\frac{\partial \varphi}{\partial t}(t, u)=\frac{\partial \varphi_{1}}{\partial t}(t, u)=f\left(t, \varphi_{1}(t, u), u\right)=f(t, \varphi(t, u), u) .
$$

Assim, temos que

$$
\left\{\begin{array}{l}
\frac{\partial \varphi}{\partial t}(t, u)=f(t, \varphi(t, u), u) \\
\varphi\left(t_{0}, u\right)=\varphi_{1}\left(t_{0}, u\right)=g(u)
\end{array}\right.
$$

Portanto, $\varphi$ é solução não-prolongável do sistema com intervalo maximal de existência $\left(m_{1}, m_{2}\right)$.

\section{3}

\section{Diferenciabilidade da Solução com respeito aos parâmetros}

Mostraremos agora que podemos também estabelecer condições para que a solução $x$ do sistema (2-3), vista no Teorema 2.1, seja não só diferenciável com respeito a $t$ mas também com relação ao próprio parâmetro $u$. Veremos que se $f \in C^{1}(G)$ e $g$ for diferenciável em $U$ então $x \in C^{1}(\Omega)$.

Teorema 2.4 Considere o sistema (2-3) e a função $x(t, u)$ contínua em $\Omega$ cuja existência é garantida pelo Teorema 2.1. Se $f \in C^{1}(G)$ e g for diferenciável em $U$ então $x \in C^{1}(\Omega)$.

Demonstração.

Notamos que, como $f \in C^{1}(G)$ então suas derivadas $\frac{\partial f_{j}}{\partial x_{i}}$ são contínuas para todo $i, j \leq n$ e, pelo Corolário A.3, $f$ é localmente Lipschitz com respeito a $x$ em $G$.

Fixemos $(t, u) \in \Omega$ (arbitrário) e sejam $\alpha \in \mathbb{R}$ tal que $0 \neq|\alpha| \leq c, k=1 \ldots m$ e o vetor canônico $e_{k} \in \mathbb{R}^{n}$, definimos:

$$
\Delta_{\alpha} x(t, u):=x\left(t, u+\alpha e_{k}\right)-x(t, u) .
$$

Como $x$ é solução em $\Omega$ então

$$
\frac{\partial x}{\partial t}\left(t, u+\alpha e_{k}\right)=f\left(t, x\left(t, u+\alpha e_{k}\right), u+\alpha e_{k}\right) .
$$

Derivando (2-29) em $t$

$$
\begin{aligned}
\frac{\partial}{\partial t}\left(\Delta_{\alpha} x\right) & =\frac{\partial x}{\partial t}\left(t, u+\alpha e_{k}\right)-\frac{\partial x}{\partial t}(t, u) \\
& =f\left(t, x\left(t, u+\alpha e_{k}\right), u+\alpha e_{k}\right)-f(t, x(t, u), u) .
\end{aligned}
$$


Sejam $y:=\left(x\left(t, u+\alpha e_{k}\right), u+\alpha e_{k}\right) \in \mathbb{R}^{n+m}$ e $z:=(x(t, u), u) \in \mathbb{R}^{n+m}$ então, pelo Lema de Hadamard A.7, existem $\psi_{i, j}(t, y, z)$ funções contínuas para todo $i \leq(n+m)$ e $j \leq n$ tais que

$$
f_{j}(t, y)-f_{j}(t, z)=\sum_{i=1}^{n+m}\left(y_{i}-z_{i}\right) \psi_{i, j}(t, y, z)
$$

$\log O$

$$
\begin{aligned}
f_{j}(t, y)-f_{j}(t, z)= & \left(\sum_{i=1}^{n}\left(x_{i}\left(t, u+\alpha e_{k}\right)-x_{i}(t, u)\right) \psi_{i, j}(t, y, z)\right) \\
& +\left(\sum_{i=1}^{m}\left[\left(u+\alpha e_{k}\right)_{i}-u_{i}\right] \psi_{(i+n), j}(t, y, z)\right) \\
= & \left(\sum_{i=1}^{n} \Delta_{\alpha} x_{i} \psi_{i, j}(t, y, z)\right)+\alpha \psi_{(k+n), j}(t, y, z) .
\end{aligned}
$$

Seja $\Psi_{j}:=\psi_{(k+n), j}$, de (2-31) e (2-33) temos para todo $j=1 \ldots n$

$$
\frac{\partial}{\partial t}\left(\Delta_{\alpha} x\right)_{j}=f_{j}(t, y)-f_{j}(t, z)=\alpha \Psi_{j}(t, y, z)+\sum_{i=1}^{n} \Delta_{\alpha} x_{i} \psi_{i, j}(t, y, z) .
$$

Como $y$ e $z$ dependem de $u$ e $\alpha$, definimos $\beta:=(u, \alpha)$ e

$$
\begin{aligned}
& A(t, \beta):=\left(\psi_{i j}(t, y, z)\right)_{i, j=1}^{n} \in \mathbb{R}^{n \times n} \quad(\text { matrix n } \times \mathrm{n}) \mathrm{e} \\
& B(t, \beta):=\left(\Psi_{j}(t, y, z)\right)_{j=1}^{n} \in \mathbb{R}^{n} .
\end{aligned}
$$

Como $\psi_{i j}$ e $\Psi_{j}$ são contínuas para todo $i, j=1 \ldots n$ então $A$ e $B$ também são funções contínuas.

De $(2-34)$ e $(2-35)$

$$
\frac{\partial}{\partial t}\left(\Delta_{\alpha} x(t, u)\right)=A(t, \beta) \cdot \Delta_{\alpha} x+\alpha B(t, \beta) .
$$

Seja $w(t, \beta):=\frac{\Delta_{\alpha} x(t, u)}{\alpha}$. Definimos o seguinte sistema :

$$
\left\{\begin{array}{l}
\frac{\partial w}{\partial t}(t, \beta)=A(t, \beta) w(t, \beta)+B(t, \beta)=: F(t, w, \beta) \\
w\left(t_{0}, \beta\right)=\frac{\Delta_{\alpha} x\left(t_{0}, u\right)}{\alpha}=\frac{g\left(u+\alpha e_{k}\right)-g(u)}{\alpha}=: G(\beta)
\end{array} .\right.
$$

Assim (2-37) é um sistema de EDO parametrizado por $\beta$ na variável $w$.

Como $\frac{\partial F}{\partial w}=A$ é contínua então, pelo Corolário A.3, $F$ é localmente Lipschitz com respeito a $\mathrm{w}$. 
Uma vez que $g$ é diferenciável em $u$, quando $\alpha \rightarrow 0$ temos que $G(u, \alpha) \rightarrow \frac{\partial g}{\partial u_{k}}(u)$. Logo $G$ pode ser prolongada por continuidade em $(u, 0)$. Assim, definimos $w\left(t_{0}, u, 0\right):=G(u, 0):=\frac{\partial g}{\partial u_{k}}(u)$ e temos que G é contínua.

Pelo Teorema de Existência e Unicidade 2.1, existe uma única função $w$ contínua que é solução de (2-37) em alguma vizinhança de $\left(t_{0}, u, 0\right)$.

Como $w(t, \beta)=w(t, u, \alpha)=\frac{\Delta_{\alpha} x(t, u)}{\alpha}$ e $w$ é contínua temos:

$$
w(t, u, 0)=\lim _{\alpha \rightarrow 0} w(t, u, \alpha)=\lim _{\alpha \rightarrow 0} \frac{x\left(t, u+\alpha e_{k}\right)-x(t, u)}{\alpha}=\frac{\partial x}{\partial u_{k}}(t, u) .
$$

Com isto, temos que existe a derivada $\frac{\partial x}{\partial u_{k}}(t, u)=w(t, u, 0) \mathrm{e}$, uma vez que $\mathrm{w}$ é contínua, $\frac{\partial x}{\partial u_{k}}$ também é contínua em $(t, u)$.

Como $(t, u)$ e $k$ são arbitrários então $\frac{\partial x}{\partial u_{k}}$ é contínua em $\Omega$ para todo $k=1 \ldots m$.

Ainda, temos também $\frac{\partial x}{\partial t}=f$ que é contínua em $G$.

Desta forma, como todas as derivadas de $x$ são contínuas em $\Omega$ temos que $x \in C^{1}(\Omega)$.

\section{4}

\section{Propriedade de Saída do Compacto}

No próximo teorema, demonstraremos uma importante propriedade da solução $\varphi$ não-prolongável definida no teorema anterior, onde garantiremos que se $K$ for um conjunto compacto contido em $G$ e $\left(t_{0}, x_{0}, u_{0}\right) \in K$, então a partir de determinado instante $T_{2}$ (no domínio de $\varphi$ ), o ponto $\left(t, \varphi\left(t, u_{0}\right), u_{0}\right.$ ) não pertence mais ao compacto $K$ para todo $t>T_{2}$.

Analogamente, também existe um instante $T_{1}$ (no domínio de $\varphi$ ) tal que o ponto $\left(t, \varphi\left(t, u_{0}\right), u_{0}\right)$ não pertence mais ao compacto $K$ para todo $t<T_{1}$. 
Teorema 2.5 Se $\left(t_{0}, x_{0}, u_{0}\right) \in K \subset G(K$ compacto $)$ e $\varphi$ é a solução nãoprolongável do Teorema 2.3 então existem $T_{1}, T_{2} \in \mathbb{R}$ em que $m_{1}<T_{1}<T_{2}<$ $m_{2}$ tais que se $t \in\left(m_{1}, T_{1}\right) \cup\left(T_{2}, m_{2}\right)$ temos que $\left(t, \varphi\left(t, u_{0}\right), u_{0}\right) \notin K$.

Demonstração.

Se $m_{2}=+\infty$, como $K$ é compacto então $K$ é limitado e existe $B_{R}$ (bola de raio R) tal que $K \subset B_{R}$.

Escolhemos $T_{2}:=R$ e se $t>T_{2}$ então

$$
\left\|\left(t, \varphi\left(t, u_{0}\right), u_{0}\right)\right\| \geq \sqrt{t^{2}+\varphi\left(t, u_{0}\right)^{2}+u_{0}^{2}} \geq t>T_{2} .
$$

$\operatorname{Logo}\left(t, \varphi\left(t, u_{0}\right), u_{0}\right) \notin K$.

Suponha então $m_{2} \in \mathbb{R}$ (finito) e seja $d_{0}:=\operatorname{dist}\{K, \partial G\}$. Como $K$ é compacto, $\partial G$ é fechado e $\partial G \cap K=\emptyset$ (pois $G$ aberto implica que $\partial G \cap G=\emptyset$ e como $K \subset G$ então $\partial G \cap K=\emptyset)$ temos, pela Proposição A.8, que $d_{0}>0$.

Seja $K^{\prime}=\left\{y \in G: \operatorname{dist}(y, K) \leq \frac{d_{0}}{2}\right\}$ onde $\operatorname{dist}(y, K):=\inf _{w \in K}\|w-y\|$.

Pela Proposição A.9, $K \subset K^{\prime} \subset G$ e $K^{\prime}$ é compacto.

Sejam $y_{1}:=\left(t_{1}, x_{1}, u_{0}\right) \in K\left(y_{1}\right.$ arbitrário $)$ e $a_{1}:=b_{1}:=\frac{d_{0}}{4}$, definimos:

$$
\Pi_{1}:=\left\{\left(t, x, u_{0}\right):\left|t-t_{1}\right| \leq a_{1},\left\|x-x_{1}\right\| \leq b_{1}\right\} .
$$

Seja $y \in \Pi_{1}$ (arbitrário) então

$$
\left\|y-y_{1}\right\| \leq\left|t-t_{1}\right|+\left\|x-x_{1}\right\| \leq \frac{d_{0}}{4}+\frac{d_{0}}{4} \leq \frac{d_{0}}{2} .
$$

Como $y_{1} \in K$

$$
\operatorname{dist}(y, K)=\inf _{w \in k}\|w-y\| \leq\left\|y_{1}-y\right\| \leq \frac{d_{0}}{2} .
$$

Logo, pela definição de $K^{\prime}, y \in K^{\prime}$ e portanto $\Pi_{1} \subset K^{\prime}$.

Uma vez que $y_{1}$ é arbitrário, temos que para todo $y_{1} \in K$ existe $\Pi_{1} \subset K^{\prime}$.

Pela Proposição A.1, existem $L_{1}$ e $L$ constantes de Lipschitz de $f$ com respeito a $x$ em $\Pi_{1}$ e $K^{\prime}$ (respectivamente). Como $\Pi_{1} \subset K^{\prime}$ então $L_{1} \leq L$. Já que $\Pi_{1}$ e $K^{\prime}$ são compactos e $f$ contínua, pelo Teorema B.28, podemos proceder como na demonstração do Teorema 2.1 e definir:

$$
\begin{gathered}
M_{1}:=\max _{y \in \Pi_{1}}\|f(y)\| \leq \max _{y \in K^{\prime}}\|f(y)\|=: M \quad \text { e } \\
h_{1}:=\min \left\{a_{1}, \frac{b_{1}}{2 M_{1}}, \frac{1}{2 L_{1}}\right\} \geq \min \left\{a_{1}, \frac{b_{1}}{2 M}, \frac{1}{2 L}\right\}=: h .
\end{gathered}
$$


Escolhendo $T_{2}:=m_{2}-h$ então $T_{2}$ não depende do ponto $y_{1}$ escolhido.

Suponha por absurdo que exista $t_{1} \in\left(T_{2}, m_{2}\right)$ tal que $\left(t_{1}, \varphi\left(t_{1}, u_{0}\right), u_{0}\right) \in K$. Considere o sistema:

$$
\left\{\begin{array}{l}
\frac{\partial x}{\partial t}\left(t, u_{0}\right)=f\left(t, x\left(t, u_{0}\right), u_{0}\right) \\
x\left(t_{1}, u_{0}\right)=\varphi\left(t_{1}, u_{0}\right)
\end{array}\right.
$$

Como $f$ é localmente Lipschitz com respeito a $x$ em $G$, pelo Teorema de Existência e Unicidade 2.1, existe uma única solução $\varphi\left(t, u_{0}\right)$ para todo $t \in\left(t_{1}-h_{1}, t_{1}+h_{1}\right) \supset\left(t_{1}-h, t_{1}+h\right)$.

Como $t_{1} \in\left(T_{2}, m_{2}\right)=\left(m_{2}-h, m_{2}\right)$ então $m_{2}-h<t_{1}$. Logo $m_{2}<t_{1}+h$.

Absurdo, pois $\left(m_{1}, m_{2}\right)$ é o intervalo maximal e $\varphi\left(t, u_{0}\right)$ não poderia ser prolongada em t até $\left(t_{1}+h\right)$. Assim, para todo $t \in\left(T_{2}, m_{2}\right)$ temos

$$
\left(t, \varphi\left(t, u_{0}\right), u_{0}\right) \notin K .
$$

Procedendo analogamente escolhendo $T_{1}:=m_{1}+h$, também temos que para todo $t \in\left(m_{1}, T_{1}\right)$

$$
\left(t, \varphi\left(t, u_{0}\right), u_{0}\right) \notin K .
$$




\section{Sistemas Lineares de EDO}

Neste capítulo estudaremos os sistemas lineares de equações diferenciais ordinárias (EDO).

Sejam $I \subseteq \mathbb{R}$ (intervalo aberto), $x_{0} \in \mathbb{R}^{n}, t_{0} \in I, b: I \rightarrow \mathbb{R}^{n}$ função contínua e $A:=\left(a_{i j}\right)_{i, j=1}^{n}$ matriz $(n \times n)$ contendo funções $a_{i j}: I \rightarrow \mathbb{R}$ contínuas. Definimos o sistema linear de EDO a seguir:

$$
\left\{\begin{array}{l}
x^{\prime}(t)=A(t) x(t)+b(t) \\
x\left(t_{0}\right)=x_{0}
\end{array} \quad \forall t \in I .\right.
$$

\section{1}

\section{Domínio de Existência e Unicidade da Solução}

Veremos a seguir que o sistema (3-1) possui uma única solução definida para todo $t \in I$, ou seja, o intervalo maximal da solução não-prolongável é $I$.

Teorema 3.1 Existe uma única solução de (3-1) definida para todo $t \in I$.

Demonstração.

Seja $f(t, x):=A(t) x(t)+b(t)$ então

$$
\frac{\partial f}{\partial x}=A(t)
$$

que é contínua (pois $a_{i j}(t)$ são contínuas para $i, j=1 \ldots n$ ) e, pelo Corolário A.3, $f$ é localmente Lipschitz com respeito a $x$.

Assim, pelo Teorema 2.3, existe única solução não-prolongável em algum intervalo $\left(m_{1}, m_{2}\right)$. Suponha por absurdo que $I=:(a, b) \neq\left(m_{1}, m_{2}\right)$, ou seja, $a \neq m_{1}$ e/ou $b \neq m_{2}$.

Como $I$ é o domínio em que estão definidas as funções $A$ e $b$ então $\left(m_{1}, m_{2}\right) \subset I$. Logo $a<m_{1}$ e/ou $b>m_{2}$. Inicialmente, suponha $b>m_{2}$. Então, podemos escolher $\beta \in\left(m_{2}, b\right)$ tal que $\left[t_{0}, m_{2}\right) \subset\left[t_{0}, \beta\right] \subset I$. 
Como $a_{i j}(t)$ são funções contínuas para $i, j=1 \ldots n$ e $\left[t_{0}, \beta\right]$ é um compacto então, pelo Teorema B.28, podemos definir:

$$
\begin{aligned}
M & :=\max _{s \in\left[t_{0}, \beta\right]}\left\|A(s) x_{0}\right\| \quad \text { (norma em } \mathbb{R}^{n} \text { ) e } \\
L & :=\max _{s \in\left[t_{0}, \beta\right]}\|A(s)\| \text { (norma matricial definida na Definição B.10). }
\end{aligned}
$$

Seja a sequência de funções $\left\{x_{k}\right\}_{k \in \mathbb{N}}$ em que $x_{k}:\left[t_{0}, \beta\right] \rightarrow \mathbb{R}^{n}$ é definida para todo $k \in \mathbb{N}$ :

$$
\begin{aligned}
x_{0}(t) & =x_{0} \\
x_{1}(t) & =x_{0}+\int_{t_{0}}^{t}\left(A(s) x_{0}+b(s)\right) d s \\
x_{2}(t) & =x_{0}+\int_{t_{0}}^{t}\left(A(s) x_{1}+b(s)\right) d s \\
& \ldots \\
x_{k}(t) & =x_{0}+\int_{t_{0}}^{t}\left(A(s) x_{k-1}+b(s)\right) d s .
\end{aligned}
$$

Seja $t \in\left[t_{0}, \beta\right]$ arbitrário então

$$
\begin{aligned}
x_{1}(t)-x_{0} & =\int_{t_{0}}^{t} A(s) x_{0} d s \\
\left\|x_{1}(t)-x_{0}\right\| & \leq\left|\int_{t_{0}}^{t}\left\|A(s) x_{0}\right\| d s\right| \leq M\left|t-t_{0}\right| \\
\left\|x_{2}(t)-x_{1}(t)\right\| & \leq\left|\int_{t_{0}}^{t}\left\|A(s)\left(x_{1}(s)-x_{0}(s)\right)\right\| d s\right|
\end{aligned}
$$

Pela Definição B.10 temos

$$
\begin{aligned}
\left\|x_{2}(t)-x_{1}(t)\right\| & \leq\left|\int_{t_{0}}^{t}\|A(s)\|\left\|\left(x_{1}(s)-x_{0}(s)\right)\right\| d s\right| \\
& \leq\left|\int_{t_{0}}^{t} L M\right| s-t_{0}|d s| \leq L M \frac{\left|t-t_{0}\right|^{2}}{2} \\
\ldots & \quad \ldots \\
\left\|x_{k}(t)-x_{k-1}(t)\right\| & \leq M L^{k-1} \frac{\left|t-t_{0}\right|^{k}}{k !}=\frac{M}{L} \frac{\left(L\left(t-t_{0}\right)\right)^{k}}{k !} \\
& \leq \frac{M}{L} \frac{\left(L\left(\beta-t_{0}\right)\right)^{k}}{k !}=: \delta_{k} \in \mathbb{R} .
\end{aligned}
$$

Para todo $k \in \mathbb{N}$ definimos $y_{k}(t):=x_{k}(t)-x_{k-1}(t)$ e temos $\left\|y_{k}(t)\right\| \leq \delta_{k}$.

Como $t$ é arbitrário e $\delta_{k}$ independe de t então $\left\|y_{k}(t)\right\| \leq \delta_{k}$ para todo $t \in\left[t_{0}, \beta\right]$. Mas, pela Definição B.21:

$$
\sum_{k=1}^{\infty} \delta_{k}=\frac{M}{L}\left(e^{L\left(\beta-t_{0}\right)}-1\right)
$$

logo, pelo Teorema de Weierstrass A.4, $\sum y_{k}$ converge uniformemente em $\left[t_{0}, \beta\right]$. 
Como

$$
x_{k}(t)=x_{0}+\sum_{i=1}^{k}\left(x_{i}(t)-x_{i-1}(t)\right)=x_{0}+\sum_{i=1}^{k} y_{i}
$$

então $x_{k}$ também converge uniformemente em $\left[t_{0}, \beta\right]$ para uma função $x(t)$.

Já que $x_{k}$ é contínua para todo $k \in \mathbb{N}$ e a convergência é uniforme, então, pelo Teorema B.45, $x$ também é contínua. De (3-4) temos

$$
x(t)=\lim _{k \rightarrow \infty} x_{k+1}(t)=x_{0}+\lim _{k \rightarrow \infty} \int_{t_{0}}^{t}\left(A(s) x_{k}(s)+b(s)\right) d s .
$$

Assim, podemos usar diretamente o Teorema B.49 e temos que quando $k \rightarrow \infty$ então $\int_{t_{0}}^{t} A(s) x_{k}(s) d s \rightarrow \int_{t_{0}}^{t} A(s) x(s) d s$. Alternativamente, podemos notar que

$$
\begin{aligned}
\left\|\int_{t_{0}}^{t} A(s)\left(x_{k}(s)-x(s)\right) d s\right\| & \leq L\left|\int_{t_{0}}^{t}\left\|x_{k}(s)-x(s)\right\| d s\right| \\
& \leq L\left|\int_{t_{0}}^{t} \max _{s \in\left[t_{0}, \beta\right]}\left\|x_{k}(s)-x(s)\right\| d s\right| \\
& \leq L\left(\beta-t_{0}\right) \max _{s \in\left[t_{0}, \beta\right]}\left\|x_{k}(s)-x(s)\right\|
\end{aligned}
$$

e como $x_{k}$ converge uniformemente em $\left[t_{0}, \beta\right]$ então, pela Definição B.41, quando $k \rightarrow \infty$

Assim, temos

$$
\max _{s \in\left[t_{0}, \beta\right]}\left\|x_{k}(s)-x(s)\right\| \rightarrow 0 .
$$

e portanto

$$
\left\|\int_{t_{0}}^{t} A(s)\left(x_{k}(s)-x(s)\right) d s\right\| \rightarrow 0
$$

$$
\int_{t_{0}}^{t} A(s) x_{k}(s) \rightarrow \int_{t_{0}}^{t} A(s) x(s)
$$

De $(3-9)$ e $(3-13)$

$$
x(t)=x_{0}+\int_{t_{0}}^{t}(A(s) x(s)+b(s)) d s .
$$

Pelo Teorema Fundamental do Cálculo B.40, $x^{\prime}(t)=A(t) x(t)+b(t)$ e como $x\left(t_{0}\right)=x_{0}$ então $x(t)$ é solução de (3-1) para todo $t \in\left[t_{0}, \beta\right] \supset\left[t_{0}, m_{2}\right)$. Absurdo, pois $\left(m_{1}, m_{2}\right)$ é o intervalo maximal de existência, logo não poderia existir solução definida para $t>m_{2}$.

Por outro lado, se $a<m_{1}$ procede-se analogamente escolhendo $\alpha \in\left(a, m_{1}\right)$ e conclui-se que existe uma solução definida para $t<m_{1}$. Absurdo.

Assim, $I=\left(m_{1}, m_{2}\right)$ e logo existe única solução definida para todo $t \in I$. 


\section{2}

\section{A Matriz Fundamental e suas propriedades}

Nesta seção consideraremos um sistema linear qualquer

$$
x^{\prime}(t)=A(t) x(t)+b(t)
$$

em que $A$ e $b$ são funções contínuas em um intervalo $I \subseteq \mathbb{R}$ e definimos:

\section{Definição 3.2 (Sistemas Homogêneos e Não-Homogêneos)}

Se para todo $t \in I$ temos $b(t)=0 \in \mathbb{R}^{n}$ então o sistema é denominado homogêneo (H). Caso contrário, o sistema é denominado não-homogêneo (NH).

\section{Definição 3.3 (Solução Geral e Particular)}

O conjunto de todas as soluções de um sistema é denominado a solução geral do sistema e dizemos que $x_{P}$ é uma solução particular deste sistema, se $x_{P}$ é uma solução qualquer conhecida do sistema.

\section{Proposição 3.4 (Espaço Vetorial de Soluções de H)}

Seja E o conjunto das soluções de um sistema homogêneo (H) então E é um espaço vetorial de dimensão $n$.

Demonstração.

Sabemos que o conjunto $F$ das funções contínuas em $I$ é um espaço vetorial (pela Definição B.2). Como $E \subset F$ mostraremos que $E$ é um subespaço vetorial. Vejamos.

1. Sejam $x$ e $y \in E$ então $x^{\prime}(t)=A(t) x(t)$ e $y^{\prime}(t)=A(t) y(t)$ para todo $t \in I$ (pelo Teorema 3.1) e logo $(x+y) \in E$ pois

$$
(x+y)^{\prime}(t)=x^{\prime}(t)+y^{\prime}(t)=A(t) x(t)+A(t) y(t)=A(t)(x+y)(t) .
$$

2. Sejam $\alpha \in \mathbb{R}$ e $x \in E$ então $(\alpha x)(t) \in E$ pois

$$
(\alpha x)^{\prime}(t)=\alpha x^{\prime}(t)=\alpha A(t) x(t)=A(t)(\alpha x)(t) .
$$

Pela Definição B.3, $E$ é um subespaço de $F$, logo $E$ é um espaço vetorial.

A seguir, definiremos uma aplicação $L: E \rightarrow \mathbb{R}^{n}$ e veremos que $L$ é um isomorfismo linear, ou seja, $L$ é uma aplicação linear bijetiva (injetiva e sobrejetiva). 
Sejam $t_{0} \in I$ (fixo) e $L: E \rightarrow \mathbb{R}^{n}$ tais que $L(x)=x\left(t_{0}\right)$ então $L$ é uma aplicação linear pois pela Definição B.55 temos

1. Se $x$ e $y \in E \Rightarrow L(x+y)=(x+y)\left(t_{0}\right)=x\left(t_{0}\right)+y\left(t_{0}\right)=L(x)+L(y)$.

2. Se $\alpha \in \mathbb{R}$ e $x \in E \Rightarrow L(\alpha x)=\alpha x\left(t_{0}\right)=\alpha L\left(x_{0}\right)$.

Seja $x_{0} \in \mathbb{R}^{n}$ arbitrário, consideremos a solução $\varphi_{1}$ do sistema:

$$
\left\{\begin{array}{l}
x^{\prime}(t)=A(t) x(t) \\
x\left(t_{0}\right)=x_{0}
\end{array} .\right.
$$

Como $\varphi_{1} \in E$ então $L\left(\varphi_{1}\right)=x_{0}$. Assim, para todo $x_{0} \in \mathbb{R}^{n}$ existe $\varphi_{1} \in E$ tal que $L\left(\varphi_{1}\right)=x_{0}, \log$ $\operatorname{Im} L=\mathbb{R}^{n}$ e $L$ é sobrejetiva (pela Proposição B.58).

Seja $\varphi_{2} \in E$ tal que $L\left(\varphi_{2}\right)=0$ então $\varphi_{2}$ é solução de

$$
\left\{\begin{array}{l}
x^{\prime}(t)=A(t) x(t) \\
x\left(t_{0}\right)=0 \in \mathbb{R}^{n}
\end{array} .\right.
$$

Como $\varphi(t)=0$ é solução trivial deste sistema então, pelo Teorema de Existência e Unicidade 3.1, $\varphi_{2}=\varphi=0$ (caso contrário existiria mais de uma solução). Com isto, Ker $L=\{0\}$ e, pelo Teorema B.57, $L$ é injetiva.

Como $L$ é injetiva e sobrejetiva então $L$ é uma aplicação linear bijetiva de $E$ em $\mathbb{R}^{n}$ e pelo Teorema B.59:

$$
\operatorname{dim} E=\operatorname{dim} \operatorname{Ker} L+\operatorname{dim} \operatorname{Im} L=0+\operatorname{dim} \mathbb{R}^{n}=n .
$$

A seguir, faremos algumas definições relevantes relacionadas aos vetores de soluções do espaço vetorial $E$ (definido na Proposição 3.4).

Definição 3.5 Sejam $\left\{\varphi^{1}, \ldots, \varphi^{n}\right\}$, n vetores quaisquer do espaço vetorial $E$. Como cada um destes vetores são funções de $I \rightarrow \mathbb{R}^{n}$, definimos uma matriz $n \times n$ como $\varphi:=\left[\begin{array}{llll}\varphi^{1} & \varphi^{2} & \ldots & \varphi^{n}\end{array}\right]$ a qual denominamos de matriz de soluções.

\section{Definição 3.6 (SFS e Matriz Fundamental)}

Se os vetores $\left\{\varphi^{1}, \ldots, \varphi^{n}\right\}$ (Definição 3.5) formam uma base do espaço $E$, denominamos estes vetores Sistema Fundamental de Soluções (SFS) e sua respectiva matriz $\varphi$ matriz fundamental do sistema. 


\section{Definição 3.7 (Wronskiano)}

Seja uma matriz de soluções $\varphi$ qualquer, denominamos de Wronskiano de $\varphi$ a função $W_{\varphi}: I \rightarrow \mathbb{R}$ tal que $W_{\varphi}(t):=\operatorname{det}(\varphi(t))$ para todo $t \in I$. Por simplicidade de notação, sempre que $\varphi$ é conhecida, denotaremos o Wronskiano de $\varphi$ como simplesmente $W:=W_{\varphi}$.

Note que, pelo Teorema B.51, a função determinante é uma função contínua e como $\varphi$ também é contínua então $W_{\varphi}$ é uma composição de funções contínuas. Logo, pelo Teorema B.30, $W_{\varphi}$ é uma função contínua.

\section{Proposição 3.8 (Solução Geral de um Sistema Homogêneo)}

A matriz $\varphi$ é uma matriz fundamental, se e somente se, a solução geral do respectivo sistema homogêneo é dada por

$$
E=\left\{\varphi c: c \in \mathbb{R}^{n}\right\} .
$$

ou simplesmente, representamos as soluções do sistema homogêneo por

$$
x_{H}(t)=\varphi(t) c \quad, \quad c \in \mathbb{R}^{n}
$$

em que c percorre todo conjunto $\mathbb{R}^{n}$.

Demonstração.

$\Rightarrow)$ Se $\varphi$ é matriz fundamental então $\left\{\varphi^{1}, \ldots, \varphi^{n}\right\}$ é um SFS, ou seja, formam uma base de E. Logo, pelo Teorema B.5, a solução geral é dada por

$$
x_{H}(t)=\sum_{i=1}^{n} c_{i} \varphi^{i}(t)=\varphi(t) c \quad, \quad c \in \mathbb{R}^{n} .
$$

$\Leftarrow)$ Se $E$ é o espaço vetorial gerado pelos n vetores $\left\{\varphi^{1}, \ldots, \varphi^{n}\right\}$ então, pela Proposição 3.4, $\operatorname{dim} E=n$. Assim, pelo Teorema B.5, temos que estes n vetores formam uma base de $E$ e logo, pela Definição 3.6, $\varphi$ é matriz fundamental.

\section{Proposição 3.9 (Solução Geral de um Sistema Não-Homogêneo)}

Sejam os sistemas lineares:

$$
x^{\prime}(t)=A(t) x(t)+b(t)(N H) \quad e \quad z^{\prime}(t)=A(t) z(t)(H) .
$$

Se $x_{p}$ é uma solução particular de (NH) então a solução geral $x_{N H}$ de (NH) é $x_{N H}=x_{H}+x_{P}$ em que $x_{H}$ é a solução geral de (H) dada pela Proposição 3.8 . 


\section{Demonstração.}

Considere um SFS $\left\{\varphi^{1}, \ldots, \varphi^{n}\right\}$ conforme Definição 3.6.

Seja $x$ solução qualquer de $(\mathrm{NH})$, definimos $z(t):=x(t)-x_{P}(t)$ e temos

$$
z^{\prime}(t)=x^{\prime}(t)-x_{P}^{\prime}(t)=(A(t) x(t)+b(t))-\left(A(t) x_{p}(t)+b(t)\right) .
$$

Logo

$$
z^{\prime}(t)=A(t)\left(x(t)-x_{P}(t)\right)=A(t) z(t) .
$$

Assim, $z$ é solução de $(\mathrm{H})$ e então $z \in E$. Com isto, existe $c \in \mathbb{R}^{n}$ tal que

$$
z(t)=\sum_{i=1}^{n} c_{i} \varphi^{i}(t)
$$

e portanto

$$
x(t)=x_{P}(t)+\sum_{i=1}^{n} c_{i} \varphi^{i}(t) .
$$

Reciprocamente, seja $c \in \mathbb{R}^{n}$ e $x(t)=x_{P}(t)+\sum_{i=1}^{n} c_{i} \varphi^{i}(t)$ então

$$
\begin{aligned}
x^{\prime}(t) & =x_{P}^{\prime}(t)+\sum_{i=1}^{n} c_{i}\left(\varphi^{i}\right)^{\prime}(t) \\
& =\left(A(t) x_{P}(t)+b(t)\right)+\sum_{i=1}^{n} c_{i} A(t) \varphi^{i}(t) \\
& =b(t)+A(t)\left(x_{P}(t)+\sum_{i=1}^{n} c_{i} \varphi^{i}(t)\right) \\
& =b(t)+A(t) x(t)
\end{aligned}
$$

Assim, temos que $x$ é solução de $(\mathrm{NH})$.

De (3-24) e (3-25) temos que a solução geral de (NH) é dada por

$$
x_{N H}(t)=x_{P}(t)+\sum_{i=1}^{n} c_{i} \varphi^{i}(t) \quad, \quad c \in \mathbb{R}^{n} .
$$

Portanto

$$
x_{N H}(t)=x_{P}(t)+x_{H}(t) .
$$

Mostraremos a seguir algumas relações entre o Wronskiano e a matriz fundamental, obtendo o importante resultado de que se existir um instante $t_{0}$ tal que $W\left(t_{0}\right) \neq 0$ então $W(t) \neq 0$ para todo $t$ e temos que $W(t)$ é o determinante de uma matriz fundamental.

Ainda, vale a recíproca, ou seja, se $W(t)$ for o determinante de uma matriz fundamental, então temos que $W(t) \neq 0$ para todo $t$. 
Teorema 3.10 Considere $W(t)$ o Wronskiano (Definição 3.7). As seguintes afirmações são equivalentes:

1. existe $t_{0} \in I$ tal que $W\left(t_{0}\right) \neq 0$;

2. $W(t) \neq 0$ para todo $t \in I$;

3. $\left\{\varphi^{1}, \ldots, \varphi^{n}\right\}$ é uma base do espaço vetorial E, ou seja, é um SFS e $\varphi$ é uma matriz fundamental do sistema.

Demonstração.

Considere a aplicação linear bijetiva $L$ definida na demonstração da Proposição 3.4. Pelo Teorema B.56, $\underline{L}$ é um isomorfismo e existe aplicação linear inversa e bijetiva $L^{-1}$ tal que, pela Definição B.55:

$$
L^{-1}\left(L\left(\varphi^{i}\right)\right)=\varphi^{i}
$$

Afirmação: $(1 \Rightarrow 3)$

Pelo Teorema B.53, como $W\left(t_{0}\right)=\operatorname{Det}\left[\varphi^{1}\left(t_{0}\right) \ldots \varphi^{n}\left(t_{0}\right)\right] \neq 0$ então $\left\{\varphi^{1}\left(t_{0}\right), \ldots, \varphi^{n}\left(t_{0}\right)\right\}$ são linearmente independentes. Por (3-28), aplicando $L^{-1}$ em $L\left(\varphi^{i}\right)=\varphi^{i}\left(t_{0}\right)$ para $i=1 \ldots n$ temos

$$
\varphi^{i}=L^{-1}\left(L\left(\varphi^{i}\right)\right)=L^{-1}\left(\varphi^{i}\left(t_{0}\right)\right)
$$

Como $L^{-1}$ é bijetiva e os $\mathrm{n}$ vetores $\left\{\varphi^{1}\left(t_{0}\right), \ldots, \varphi^{n}\left(t_{0}\right)\right\}$ são linearmente independentes então os $\mathrm{n}$ vetores $\left\{\varphi^{1}, \ldots, \varphi^{n}\right\}$ também são linearmente independentes. Assim, pelo Teorema B.5, $\left\{\varphi^{1}, \ldots, \varphi^{n}\right\}$ formam uma base de $E$.

Afirmação: $(3 \Rightarrow 2)$

Pelo Teorema B.5, $\left\{\varphi^{1}, \ldots, \varphi^{n}\right\}$ são linearmente independentes.

Logo, pela Definição B.4:

$$
\sum_{i=1}^{n} c_{i} \varphi^{i}=0 \in E \text { (função identicamente nula) } \Rightarrow c_{i}=0 \quad i=1 \ldots n .
$$

Portanto

$$
\sum_{i=1}^{n} c_{i} \varphi^{i}(t)=0 \quad \forall t \in I \Rightarrow c_{i}=0 \quad i=1 \ldots n .
$$

Assim, pela Definição B. $4,\left\{\varphi^{1}(t), \ldots, \varphi^{n}(t)\right\}$ são linearmente independentes para todo $t \in I$. Pelo Teorema B.53, $W(t) \neq 0$ para todo $t \in I$.

Afirmação: $(2 \Rightarrow 1)$

Se $W(t) \neq 0$ para todo $t \in I$ então, trivialmente, existe $t_{0} \in I$ tal que $W\left(t_{0}\right) \neq 0$. 
A seguir, mostraremos a relação entre o Wronskiano, sua derivada e o traço da matriz $A(t)$, bem como veremos que podemos calculá-lo usando uma integral.

\section{Teorema 3.11 (Fórmula de Liouville)}

Seja W(t) o Wronskiano (Definição 3.7) então

$$
W^{\prime}(t)=\operatorname{tr}(A(t)) W(t) .
$$

Demonstração.

Sejam

$$
\omega_{j}:=\left[\begin{array}{ccc}
\varphi_{1}^{1} & \ldots & \varphi_{1}^{n} \\
\ldots & \ldots & \ldots \\
\varphi_{j-1}^{1} & \ldots & \varphi_{j-1}^{n} \\
\left(\varphi_{j}^{1}\right)^{\prime} & \ldots & \left(\varphi_{j}^{n}\right)^{\prime} \\
\varphi_{j+1}^{1} & \ldots & \varphi_{j+1}^{n} \\
\ldots & \ldots & \ldots \\
\varphi_{n}^{1} & \ldots & \varphi_{n}^{n}
\end{array}\right]=:\left[\begin{array}{c}
\varphi_{1} \\
\ldots \\
\varphi_{j-1} \\
\left(\varphi_{j}\right)^{\prime} \\
\varphi_{j}^{j+1} \\
\ldots \\
\varphi_{n}
\end{array}\right] j=1 \ldots n .
$$

Assim, pelo Teorema A.10 (Derivada de Determinantes) temos

$$
W^{\prime}(t)=\sum_{j=1}^{n} \operatorname{det}\left(\omega_{j}(t)\right)=\sum_{j=1}^{n}\left|\omega_{j}(t)\right|
$$

Como

$$
\left(\varphi^{i}\right)^{\prime}(t)=\frac{\mathrm{d} \varphi^{i}}{\mathrm{~d} t}(t)=A(t) \varphi^{i}(t) \Rightarrow\left(\varphi_{j}^{i}\right)^{\prime}(t)=\frac{\mathrm{d} \varphi_{j}^{i}}{\mathrm{~d} t}(t)=\sum_{k=1}^{n} a_{j k} \varphi_{k}^{i}(t)
$$

então

$$
\begin{aligned}
& \left(\varphi_{j}^{1}\right)^{\prime}=a_{j 1} \varphi_{1}^{1}+\cdots+a_{j n} \varphi_{n}^{1} \\
& \ldots \ldots \\
& \left(\varphi_{j}^{j}\right)^{\prime}=a_{j 1} \varphi_{1}^{j}+\cdots+a_{j n} \varphi_{n}^{j} \\
& \cdots \cdots \\
& \left(\varphi_{j}^{n}\right)^{\prime}=a_{j 1} \varphi_{1}^{j}+\cdots+a_{j n} \varphi_{n}^{n}
\end{aligned}
$$

Rescrevendo 3-35 temos

$$
\begin{aligned}
& {\left[\begin{array}{c}
\left(\varphi_{j}^{1}\right)^{\prime} \\
\ldots \\
\left(\varphi_{j}^{j}\right)^{\prime} \\
\ldots \\
\left(\varphi_{j}^{n}\right)^{\prime}
\end{array}\right]^{t}=a_{j 1}\left[\begin{array}{c}
\varphi_{1}^{1} \\
\ldots \\
\varphi_{1}^{j} \\
\cdots \\
\varphi_{1}^{n}
\end{array}\right]^{t}+\cdots+a_{j j}\left[\begin{array}{c}
\varphi_{j}^{1} \\
\cdots \\
\varphi_{j}^{j} \\
\cdots \\
\varphi_{j}^{n}
\end{array}\right]^{t}+\cdots+a_{j n}\left[\begin{array}{c}
\varphi_{n}^{1} \\
\cdots \\
\varphi_{n}^{j} \\
\cdots \\
\varphi_{n}^{n}
\end{array}\right]^{t}} \\
& \Rightarrow\left(\varphi_{j}\right)^{\prime}=a_{j 1} \varphi_{1}+\cdots+a_{j j} \varphi_{j}+\cdots+a_{j n} \varphi_{n}=\sum_{k=1}^{n} a_{j k} \varphi_{k} .
\end{aligned}
$$


Seja $D$ a função determinante (definida no Teorema B.51) então

$$
\begin{aligned}
\left|\omega_{j}\right| & =D\left(\varphi_{1}, \ldots, \varphi_{j-1}, \quad\left(\varphi_{j}\right)^{\prime} \quad, \varphi_{j+1}, \ldots, \varphi_{n}\right) \\
& =D\left(\varphi_{1}, \ldots, \varphi_{j-1}, \quad \sum_{k=1}^{n} a_{j k} \varphi_{k} \quad, \varphi_{j+1}, \ldots, \varphi_{n}\right) .
\end{aligned}
$$

Pela multilinearidade (Definição B.50) da função determinante temos

$$
\left|\omega_{j}\right|=\sum_{k=1}^{n} a_{j k} D\left(\varphi_{1}, \ldots, \varphi_{j-1}, \varphi_{k}, \varphi_{j+1}, \ldots, \varphi_{n}\right) .
$$

Como para todo $k \neq j$ a linha $\varphi_{k}$ se repete para algum $\varphi_{j}$ então

$$
D\left(\varphi_{1}, \ldots, \varphi_{j-1}, \varphi_{k}, \varphi_{j+1}, \ldots, \varphi_{n}\right)=0 \quad \forall k \neq j .
$$

Assim, temos

$$
\left|\omega_{j}\right|=a_{j j} D\left(\varphi_{1}, \ldots, \varphi_{j-1}, \varphi_{j}, \varphi_{j+1}, \ldots, \varphi_{n}\right)=a_{j j} D(\varphi)=a_{j j} W .
$$

De $(3-33)$ e $(3-40)$

$$
W^{\prime}(t)=\sum_{j=1}^{n}\left|\omega_{j}(t)\right|=\sum_{j=1}^{n} a_{j j}(t) W(t)=\operatorname{tr}(A(t)) W(t) .
$$

Corolário 3.12 Para todo $t \in I$ temos

$$
W(t)=W\left(t_{0}\right) e^{\int_{t_{0}}^{t} \operatorname{tr}(A(s)) d s} .
$$

\section{Demonstração.}

Se existir $t_{0} \in I$ tal que $W\left(t_{0}\right)=0$ temos, pelo Teorema 3.10, que $W(t)=0$ para todo $t \in I$ e logo a expressão é trivialmente válida.

Se $W(t) \neq 0$ para todo $t \in I$ então

$$
\frac{W^{\prime}(t)}{W(t)}=\operatorname{tr}(A(t))
$$

Integrando de $t_{0}$ a $t$ temos

$$
\int_{t_{0}}^{t} \frac{W^{\prime}(s)}{W(s)} d s=\ln \left|\frac{W(t)}{W\left(t_{0}\right)}\right|=\int_{t_{0}}^{t} \operatorname{tr}(A(s)) d s .
$$

$\log 0$

$$
|W(t)|=\left|W\left(t_{0}\right)\right| e^{\int_{t_{0}}^{t} \operatorname{tr}(A(s)) d s} .
$$

Como $W(t)$ é uma função contínua e $W(t) \neq 0$ para todo $t \in I$ então $W(t)$ não muda de sinal, ou seja, $W(t)>0$ para todo $t \in I$ ou $W(t)<0$ para todo $t \in I$. Portanto

$$
W(t)=W\left(t_{0}\right) e^{\int_{t_{0}}^{t} t r(A(s)) d s} .
$$




\section{3}

\section{Solução de Sistemas Lineares com coeficientes variáveis}

O teorema a seguir nos fornece a solução do sistema (3-1) como função de uma matriz fundamental.

Teorema 3.13 Seja o sistema (3-1) e $\varphi(t)$ a respectiva matriz fundamental, então a solução é dada por

$$
x(t)=\varphi(t)\left(\varphi^{-1}\left(t_{0}\right) x_{0}+\int_{t_{0}}^{t} \varphi^{-1}(s) b(s) d s\right)
$$

em que $\varphi^{-1}(t)$ é a matriz inversa de $\varphi(t)$ definida para todo $t \in I$.

Demonstração.

Como $\varphi(t)$ é uma matriz fundamental então pelo Teorema 3.10

$$
\operatorname{det}(\varphi(t))=|\varphi(t)| \neq 0 \text { para todo } t \in I \text {. }
$$

Assim, pelo Teorema B.53, a matriz $\varphi(t)$ é inversível para todo $t \in I$ e logo existe matriz inversa $\varphi^{-1}(t)$ definida para todo $t \in I$.

Seja $d: I \rightarrow \mathbb{R}^{n}$ tal que $\varphi(t) d^{\prime}(t)=b(t)$ então pela definição de matriz inversa (Teorema B.53)

$$
\varphi^{-1}(t) b(t)=\varphi^{-1}(t) \varphi(t) d^{\prime}(t) \Rightarrow \varphi^{-1}(t) b(t)=d^{\prime}(t) .
$$

Como $\varphi$ é contínua temos, pela Proposição A.11, que $\varphi^{-1}$ também é contínua. Já que $b$ e $\varphi^{-1}$ são contínuas então pelo Teorema Fundamental do Cálculo B.40

$$
d(t)=d\left(t_{0}\right)+\int_{t_{0}}^{t} \varphi^{-1}(s) b(s) d s .
$$

Logo, $d$ está bem definida para todo $t \in I$. Como $\varphi(t) \cdot d^{\prime}(t)=b(t)$ então

$$
\begin{gathered}
{\left[\begin{array}{ccc}
\varphi_{1}^{1}(t) & \ldots & \varphi_{1}^{n}(t) \\
\ldots & \ldots & \ldots \\
\varphi_{n}^{1}(t) & \ldots & \varphi_{n}^{n}(t)
\end{array}\right]\left[\begin{array}{c}
d_{1}^{\prime}(t) \\
\ldots \\
d_{n}^{\prime}(t)
\end{array}\right]=\left[\begin{array}{c}
\sum_{i=1}^{n} \varphi_{1}^{i}(t) d_{i}^{\prime}(t) \\
\ldots \\
\sum_{i=1}^{n} \varphi_{n}^{i}(t) d_{i}^{\prime}(t)
\end{array}\right]=\left[\begin{array}{c}
b_{1}^{\prime}(t) \\
\ldots \\
b_{n}^{\prime}(t)
\end{array}\right]} \\
\Rightarrow b_{j}(t)=\sum_{i=1}^{n} \varphi_{j}^{i}(t) d_{i}^{\prime}(t) \quad j=1 \ldots n \Rightarrow b(t)=\sum_{i=1}^{n} \varphi^{i}(t) d_{i}^{\prime}(t) .
\end{gathered}
$$

Pela definição de matriz fundamental (Definição 3.6) temos que $\varphi^{i}$ é uma solução do sistema homogêneo $x^{\prime}(t)=A(t) x(t)$ para $i=1 \ldots n$, logo

$$
\left(\varphi^{i}\right)^{\prime}(t)=A(t) \varphi^{i}(t) \quad i=1 \ldots n
$$


Multiplicando (3-51) por $d_{i}(t)$ para $i=1 \ldots n$

$$
\begin{aligned}
\left(\varphi^{i}\right)^{\prime}(t) d_{i}(t) & =A(t) \varphi^{i}(t) d_{i}(t) \\
\sum_{i=1}^{n}\left(\varphi^{i}\right)^{\prime}(t) d_{i}(t) & =A(t) \sum_{i=1}^{n} \varphi^{i}(t) d_{i}(t) .
\end{aligned}
$$

Seja $y(t):=\varphi(t) d(t)=\sum_{i=1}^{n} \varphi^{i}(t) d_{i}(t)$ então, aplicando a regra da soma e produto das derivadas (Teorema B.39)

$$
y^{\prime}(t)=\sum_{i=1}^{n}\left(\varphi^{i}\right)^{\prime}(t) d_{i}(t)+\sum_{i=1}^{n} \varphi^{i}(t) d_{i}^{\prime}(t) .
$$

De $(3-52)$ e $(3-50)$

$$
y^{\prime}(t)=A(t) y(t)+b(t) .
$$

Pela Definição 3.3, $x_{P}(t):=y(t)=\varphi(t) \cdot d(t)$ é solução particular do sistema não-homogêneo e, pela Proposição 3.9, a solução geral deste sistema é

$$
\begin{aligned}
x & =x_{H}+x_{P} \\
x(t) & =\varphi(t)(c+d(t)) \quad, \quad c \in \mathbb{R}^{n}
\end{aligned}
$$

De $(3-48)$

$$
x(t)=\varphi(t)\left(c+d\left(t_{0}\right)+\int_{t_{0}}^{t} \varphi^{-1}(s) b(s) d s\right) \quad, \quad c \in \mathbb{R}^{n}
$$

Considerando a condição inicial $x\left(t_{0}\right)=x_{0}$ temos que

$$
x_{0}=x\left(t_{0}\right)=\varphi\left(t_{0}\right)\left(c+d\left(t_{0}\right)\right) \Rightarrow c+d\left(t_{0}\right)=\varphi^{-1}\left(t_{0}\right) x_{0} .
$$

De (3-56) e (3-57) temos que a solução do sistema (3-1) é dada por

$$
x(t)=\varphi(t)\left(\varphi^{-1}\left(t_{0}\right) x_{0}+\int_{t_{0}}^{t} \varphi^{-1}(s) b(s) d s\right) .
$$

\section{4}

\section{A Exponencial Matricial}

Nesta seção, definiremos a exponencial de uma matriz e apresentaremos suas principais propriedades, relevantes ao presente estudo.

Definição 3.14 Seja A uma matriz (real ou complexa) $(n \times n)$, definimos a exponencial de A como:

$$
e^{A}=\sum_{k=0}^{\infty} \frac{A^{k}}{k !} \quad \text { em que } A^{0}:=I \text { (matriz identidade). }
$$


Usando a norma matricial induzida (Definição B.10) e suas propriedades:

$$
\sum_{k=0}^{\infty}\left\|\frac{A^{k}}{k !}\right\|=\sum_{k=0}^{\infty} \frac{\left\|A^{k}\right\|}{k !} \leq \sum_{k=0}^{\infty} \frac{\|A\|^{k}}{k !}=e^{\|A\|} \in \mathbb{R} .
$$

Pelo Teorema B.20 temos que a série é absolutamente convergente, logo convergente e portanto $e^{A}$ está bem definido. Note que, no caso de matrizes complexas, usamos este teorema considerando a bijeção entre as matrizes complexas $\mathbb{C}^{n \times n}$ e os vetores reais $\mathbb{R}^{2 n^{2}}$.

No teorema a seguir, usaremos o conceito de derivada de uma função matricial (Definição B.32).

\section{Teorema 3.15 (Diferenciabilidade)}

Seja A matriz real e $\varphi(t)=e^{t A}$ então $\varphi \in C^{\infty}(\mathbb{R})$ e para todo $k \in \mathbb{N}$ temos $\varphi^{(k)}=A^{k} e^{t A}$ em que $\varphi^{(k)}$ é a derivada de ordem $k$ de $\varphi$.

Demonstração.

Mostraremos primeiramente que $\varphi$ é contínua em $\mathbb{R}$.

Seja a sequência de funções matriciais $f_{k}$ definida para todo $k \in \mathbb{N}$

$$
f_{k}(t)=\frac{t^{k} A^{k}}{k !} \Rightarrow e^{t A}=I+\sum_{k=1}^{\infty} f_{k}(t) \text {. }
$$

Seja $t_{1} \in \mathbb{R}$ e $k \in \mathbb{N}$ (fixos e arbitrários), usando a norma matricial (Definição B.10) e suas propriedades, temos para todo $t \in \mathbb{R}$

$$
\left\|f_{k}(t)-f_{k}\left(t_{1}\right)\right\|=\left\|\frac{A^{k}}{k}\left(t^{k}-t_{1}^{k}\right)\right\| \leq \frac{\left\|A^{k}\right\|}{k !}\left|t^{k}-t_{1}^{k}\right| .
$$

Como $\frac{\left\|A^{k}\right\|}{k !} \in \mathbb{R}$ e $\lim _{t \rightarrow t_{1}}\left|t^{k}-t_{1}^{k}\right|=0$ então pelo Teorema B.25

$$
\lim _{t \rightarrow t_{1}}\left\|f_{k}(t)-f_{k}\left(t_{1}\right)\right\|=0 \Rightarrow \lim _{t \rightarrow t_{1}} f_{k}(t)=f_{k}\left(t_{1}\right) .
$$

Assim, temos que $f_{k}$ é contínua em $t_{1} \in \mathbb{R}$. Como $t_{1}$ e $k$ são arbitrários então $f_{k}$ é contínua em $\mathbb{R}$ para todo $k \in \mathbb{N}$.

Seja $M>0$ (arbitrário) e $t \in[-M, M]$, fixamos $t_{1}=0$ em (3-61)

$$
\left\|f_{k}(t)\right\| \leq M^{k} \frac{\left\|A^{k}\right\|}{k !} \leq \frac{\|M A\|^{k}}{k !}=: \alpha_{k} \in \mathbb{R} .
$$

Pela Definição B.21, $\sum_{k=1}^{\infty} \alpha_{k}=\left(e^{\|M A\|}-1\right)$ e logo, pelo Teorema de Weierstrass A.4, a série $\sum f_{k}$ converge uniformemente em $[-M, M]$. 
Como $f_{k}$ é contínua em $t$ para todo $k \in \mathbb{N}$ então, pelo Teorema B.46, $\sum f_{k}$ também é contínua em $t \in[-M, M]$. Mas $t$ e $M$ são arbitrários, logo $f(t):=\sum_{k=1}^{\infty} f_{k}(t)$ é contínua para todo $t \in \mathbb{R}$.

De (3-60) temos que $e^{t A}=I+f(t), \operatorname{logo} e^{t A}$ também é contínua em $\mathbb{R}$.

A seguir, mostraremos que $\varphi$ é diferenciável em $\mathbb{R}$.

Procedendo analogamente, seja $M>0$ (arbitrário) e $t \in[-M, M]$ temos

$$
f_{k}(t)=\frac{t^{k} A^{k}}{k !} \Rightarrow f_{k}^{\prime}(t)=\frac{t^{k-1} A^{k}}{k !} k=A \frac{(t A)^{k-1}}{(k-1) !} .
$$

Usando a norma matricial (Definição B.10) e suas propriedades temos

$$
\left\|f_{k}^{\prime}(t)\right\|=\left\|A \frac{(t A)^{k-1}}{(k-1) !}\right\| \leq\|A\| \frac{\|t A\|^{k-1}}{(k-1) !} \leq\|A\| \frac{\|M A\|^{k-1}}{(k-1) !}=: \beta_{k} \in \mathbb{R} .
$$

Novamente, como a série $\sum \beta_{k}$ é convergente, temos que a série $\sum f_{k}^{\prime}$ converge uniformemente em $[-M, M]$. Ainda, para todo $k \in \mathbb{N}$ temos que $f_{k}(0)=0$ e $\log \mathrm{\sum} f_{k}(0)=0$.

Assim, pelo Teorema B.48, temos que a série $\sum f_{k}$ converge uniformemente em $[-M, M]$ para uma função $f$ diferenciável tal que

$$
\left(e^{t A}\right)^{\prime}=(I+f(t))^{\prime}=f^{\prime}(t)=\sum_{k=1}^{\infty} f_{k}^{\prime}(t) .
$$

De $(3-64)$

$$
\varphi^{\prime}(t)=\left(e^{t A}\right)^{\prime}=\sum_{k=1}^{\infty} A \frac{(t A)^{k-1}}{(k-1) !}=A \sum_{k=0}^{\infty} \frac{(t A)^{k}}{k !}=A e^{t A}=A \varphi(t) .
$$

Como $\varphi^{\prime}$ é o produto de funções contínuas $\varphi$ e $A$ (constante) então, pelo Teorema B.30, $\varphi^{\prime}$ é contínua em $[-M, M]$. Mas $M$ é arbitrário, logo $\varphi \in C^{1}(\mathbb{R})$.

Por indução em $k$, mostraremos que $\varphi \in C^{k}(\mathbb{R})$ e $\varphi^{(k)}(t)=A^{k} e^{t A}$ para todo $k \in \mathbb{N}$. Vimos que é válido para $k=1$. Suponha válido para $k \in \mathbb{N}$.

$$
\varphi^{(k+1)}(t)=\left(\varphi^{(k)}(t)\right)^{\prime}=\left(A^{k} e^{t A}\right)^{\prime}=A^{k}\left(e^{t A}\right)^{\prime}=A^{k}\left(A e^{t A}\right)=A^{k+1} e^{t A} .
$$

Como $A^{k+1}$ é constante e $e^{t A}$ é contínua, temos que $\varphi^{(k+1)}$ é contínua, logo $\varphi \in C^{k+1}(\mathbb{R})$. Assim, $\varphi^{(k)}(t)=A^{k} e^{t A}$ para todo $k \in \mathbb{N}$ e $\varphi \in C^{\infty}(\mathbb{R})$. 


\section{Proposição 3.16 (Comutatividade)}

Sejam $A$ e $B$ matrizes (reais ou complexas) tais que $A B=B A$ então

$$
B e^{t A}=e^{t A} B .
$$

Demonstração.

Se $A$ e $B$ comutam então, indutivamente, temos

$$
B A^{k}=B A A^{k-1}=A B A^{k-1}=A B A A^{k-2}=A^{2} B A^{k-2}=\cdots=A^{k} B .
$$

Logo, pela Definição $3.14, B e^{t A}=e^{t A} B$.

\section{Proposição 3.17 (Produto de Exponenciais Matriciais)}

Sejam $A$ e $B$ matrizes (reais ou complexas) tais que $A B=B A$ então

$$
e^{(A+B)}=e^{A} e^{B}
$$

Demonstração.

Inicialmente, suponha $A$ e $B$ matrizes reais. Pelo Teorema 3.15 temos

$$
\left(e^{t A}\right)^{\prime}=A e^{t A} \quad e \quad\left(e^{t B}\right)^{\prime}=B e^{t B} .
$$

Como $A$ e $B$ comutam temos pela Proposição 3.16

$$
e^{t A} B=B e^{t A}
$$

Consideremos o sistema linear de EDO definido para todo $t \in \mathbb{R}$

$$
\left\{\begin{array}{l}
x^{\prime}(t)=(A+B) x(t) \\
x(0)=x_{0} \in \mathbb{R}^{n}
\end{array} .\right.
$$

Seja $y(t)=e^{t A} e^{t B} x_{0}$ e $z(t)=e^{t(A+B)} x_{0}$. Derivando $y$ e $z$ em $t$ temos, pela regra do produto das derivadas (Teorema B.39), de (3-70) e (3-71) temos

$$
\begin{aligned}
y^{\prime}(t) & =A e^{t A} e^{t B} x_{0}+e^{t A} B e^{t B} x_{0}=A e^{t A} e^{t B} x_{0}+B e^{t A} e^{t B} x_{0} \\
& =(A+B) e^{t A} e^{t B} x_{0}=(A+B) y(t) \\
z^{\prime}(t) & =(A+B) e^{t(A+B)} x_{0}=(A+B) z(t) .
\end{aligned}
$$

Assim $y$ e $z$ são soluções de (3-72). Pelo Teorema de Existência e Unicidade 3.1 temos que $x:=y=z$ é a única solução definida para todo $t \in \mathbb{R}$ tal que

$$
x(t)=e^{t A} e^{t B} x_{0}=e^{t(A+B)} x_{0} .
$$

Escolhendo $t=1$ e como $x_{0}$ é arbitrário temos

$$
e^{A} e^{B} x_{0}=e^{(A+B)} x_{0} \Rightarrow e^{A} e^{B}=e^{(A+B)}
$$


Se $A$ e $B$ forem complexas então, pela Proposição B.54, existem matrizes reais equivalentes $A_{\mathbb{R}}$ e $B_{\mathbb{R}}$ e usamos o resultado anterior obtendo

$$
e^{A_{\mathbb{R}}} e^{B_{\mathbb{R}}}=e^{\left(A_{\mathbb{R}}+B_{\mathbb{R}}\right)}=e^{(A+B)_{\mathbb{R}}} .
$$

Pela equivalência entre as matrizes complexas $A$ e $B$ e suas respectivas representações reais $A_{\mathbb{R}}$ e $B_{\mathbb{R}}$ dadas pela Proposição B.54 temos

$$
e^{A} e^{B}=e^{A+B} .
$$

\section{Proposição 3.18 (Inversa da Exponencial Matricial)}

A matriz $e^{A}$ é inversível para qualquer matriz (real ou complexa) $A(n \times n) e$ sua inversa é dada por

$$
\left(e^{A}\right)^{-1}=e^{-A}
$$

Demonstração.

Pela Definição 3.14, temos que $e^{0}=I$ (onde 0 é a matriz nula $n \times n$ ).

Como $A$ e $-A$ comutam, ou seja, $A(-A)=(-A) A$ então pela Proposição 3.17

$$
I=e^{0}=e^{A-A}=e^{A} e^{-A} \Rightarrow\left(e^{A}\right)^{-1}=e^{-A} .
$$

Proposição 3.19 Sejam $S$, A e B matrizes (reais ou complexas) tal que $S$ é inversivel e $A=S^{-1} B S$ então

$$
e^{t A}=S^{-1} e^{t B} S
$$

Demonstração.

Por indução em $k$ mostraremos que

$$
A^{k}=S^{-1} B^{k} S \quad \forall k \in \mathbb{N} .
$$

Se $k=1$ então $A^{1}=A=S^{-1} B S$, validando a base de indução. Supondo válido para $(k-1)$ que $A^{k-1}=S^{-1} B^{k-1} S$ e considerando que $S S^{-1}=I$

$$
A^{k}=A^{k-1} A=\left(S^{-1} B^{k-1} S\right)\left(S^{-1} B S\right)=S^{-1} B^{k} S .
$$

Logo $A^{k}=S^{-1} B^{k} S$ para todo $k \in \mathbb{N}$ e portanto é válido para todo $k \in \mathbb{N}$ que

$$
I+t A+\frac{t^{2} A^{2}}{2 !}+\cdots+\frac{t^{k} A^{k}}{k !}=S^{-1}\left(I+t B+\frac{t^{2} B^{2}}{2 !}+\cdots+\frac{t^{k} B^{k}}{k !}\right) S .
$$

Pela Definição 3.14, quando $k \rightarrow \infty$ temos

$$
e^{t A}=S^{-1} e^{t B} S
$$


A seguir, mostraremos como podemos calcular a exponencial de uma matriz qualquer. Iniciaremos com o caso de uma matriz diagonal, depois usamos o resultado para tratar o caso de um bloco de Jordan que por sua vez nos permite calcular a exponencial de uma matriz de Jordan. Por fim, usamos este último resultado e o Teorema de Jordan B.63 para calcular a exponencial de uma matriz qualquer.

\section{Proposição 3.20 (Exponencial de Matrizes Diagonais)}

Se A é uma Matriz diagonal $(n \times n)$ tal que

$$
A=\left[\begin{array}{ccc}
\lambda_{1} & & 0 \\
& \ddots & \\
0 & & \lambda_{n}
\end{array}\right] \quad \text { então } \quad e^{t A}=\left[\begin{array}{ccc}
e^{\lambda_{1}} t & & 0 \\
& \ddots & \\
0 & & e^{\lambda_{n} t}
\end{array}\right]
$$

Demonstração.

$$
A^{2}=\left[\begin{array}{ccc}
\lambda_{1}^{2} & & 0 \\
& \ddots & \\
0 & & \lambda_{n}^{2}
\end{array}\right] \Rightarrow \cdots \Rightarrow A^{k}=\left[\begin{array}{ccc}
\lambda_{1}^{k} & & 0 \\
& \ddots & \\
0 & & \lambda_{n}^{k}
\end{array}\right] \quad \forall k \in \mathbb{N} .
$$

Pela Definição 3.14

$$
e^{t A}=\sum_{k=0}^{\infty} \frac{(t A)^{k}}{k !}=I+t A+\frac{t^{2} A^{2}}{2 !}+\frac{t^{3} A^{3}}{3 !}+\ldots
$$

De $(3-83)$ e $(3-84)$

$$
e^{t A}=\left[\begin{array}{ccc}
1+\lambda_{1} t+\frac{\lambda_{1}^{2} t^{2}}{2 !}+\frac{\lambda_{1}^{3} t^{3}}{3 !}+\ldots & 0 \\
0 & \ddots & \\
& & 1+\lambda_{n} t+\frac{\lambda_{n}^{2} t^{2}}{2 !}+\frac{\lambda_{n}^{3} t^{3}}{3 !}+\ldots
\end{array}\right]
$$

Logo

$$
e^{t A}=\left[\begin{array}{ccc}
e^{\lambda_{1} t} & & 0 \\
& \ddots & \\
0 & & e^{\lambda_{n} t}
\end{array}\right]
$$




\section{Proposição 3.21 (Exponencial de Blocos de Jordan)}

Se A é um bloco de Jordan $J(n \times n)$, ou seja

$$
A=J=\left[\begin{array}{cccc}
\lambda & 1 & & 0 \\
& \ddots & \ddots & \\
& & \lambda & 1 \\
0 & & & \lambda
\end{array}\right] \quad \text { então } \quad e^{t A}=e^{\lambda t}\left[\begin{array}{cccccc}
1 & t & \frac{t^{2}}{2 !} & \frac{t^{3}}{3 !} & \ldots & \frac{t^{n-1}}{(n-1) !} \\
0 & 1 & t & \frac{t^{2}}{2 !} & \ddots & \vdots \\
0 & 0 & 1 & t & \ddots & \frac{t^{3}}{3 !} \\
0 & 0 & 0 & 1 & \ddots & \frac{t^{2}}{2 !} \\
\vdots & \vdots & \vdots & \ddots & \ddots & t \\
0 & 0 & 0 & 0 & \ldots & 1
\end{array}\right]
$$

Demonstração.

$$
A=\left[\begin{array}{llll}
\lambda & & & 0 \\
& \ddots & & \\
& & \ddots & \\
0 & & & \lambda
\end{array}\right]+\left[\begin{array}{cccc}
0 & 1 & & 0 \\
& \ddots & \ddots & \\
& & \ddots & 1 \\
0 & & & 0
\end{array}\right]=: \lambda I+N .
$$

A cada multiplicação $N . N$, a diagonal com 1 se desloca um nível para cima e temos $N^{n}=0$. Assim, temos que

$$
e^{t N}=\sum_{k=0}^{\infty} \frac{(t N)^{k}}{k !}=\sum_{k=0}^{n-1} \frac{(t N)^{k}}{k !}=I+t N+\frac{t^{2} N^{2}}{2 !}+\frac{t^{3} N^{3}}{3 !}+\cdots+\frac{t^{n-1} N^{n-1}}{(n-1) !}
$$

Com isto, temos

$$
e^{t N}=\left[\begin{array}{cccccc}
1 & t & \frac{t^{2}}{2 !} & \frac{t^{3}}{3 !} & \cdots & \frac{t^{n-1}}{(n-1) !} \\
0 & 1 & t & \frac{t^{2}}{2 !} & \ddots & \vdots \\
0 & 0 & 1 & t & \ddots & \frac{t^{3}}{3 !} \\
0 & 0 & 0 & 1 & \ddots & \frac{t^{2}}{2 !} \\
\vdots & \vdots & \vdots & \ddots & \ddots & t \\
0 & 0 & 0 & 0 & \cdots & 1
\end{array}\right]
$$

Como $(\lambda I t)$ e $(N t)$ comutam então pela Proposição 3.17

$$
e^{t A}=e^{(\lambda I+N) t}=e^{\lambda I t} e^{N t}
$$

Como $e^{\lambda I t}$ é uma matriz diagonal temos pela Proposição 3.20

$$
e^{t A}=\left[\begin{array}{ccc}
e^{\lambda t} & & 0 \\
& \ddots & \\
0 & & e^{\lambda t}
\end{array}\right] e^{t N}=e^{\lambda t} e^{t N}
$$




\section{Proposição 3.22 (Exponencial de Matrizes de Jordan)}

Se A é uma Matriz de Jordan $(n \times n)$ (definida no Teorema B.63) com $k$ blocos de Jordan $J_{1}, \ldots, J_{k}$ (definidos na Proposição 3.21), ou seja, $i=1 \ldots k$ :

$$
\begin{aligned}
J_{i}= & {\left[\begin{array}{cccc}
\lambda_{i} & 1 & & 0 \\
& \ddots & \ddots & \\
& & \lambda_{i} & 1 \\
0 & & & \lambda_{i}
\end{array}\right] \quad A=\left[\begin{array}{ccc}
J_{1} & & 0 \\
& \ddots & \\
0 & & J_{k}
\end{array}\right] \text { então } e^{t A}=\left[\begin{array}{ccc}
e^{t J_{1}} & & 0 \\
& \ddots & \\
0 & & e^{t J_{k}}
\end{array}\right] } \\
& \text { (cada um dos blocos } \left.J_{i} \text { tem dimensão } n_{i} \times n_{i} \text { tal que } \sum_{i=1}^{k} n_{i}=n\right) .
\end{aligned}
$$

Demonstração. Seja $A_{i}$ a Matriz contendo apenas o bloco $J_{i}$, ou seja:

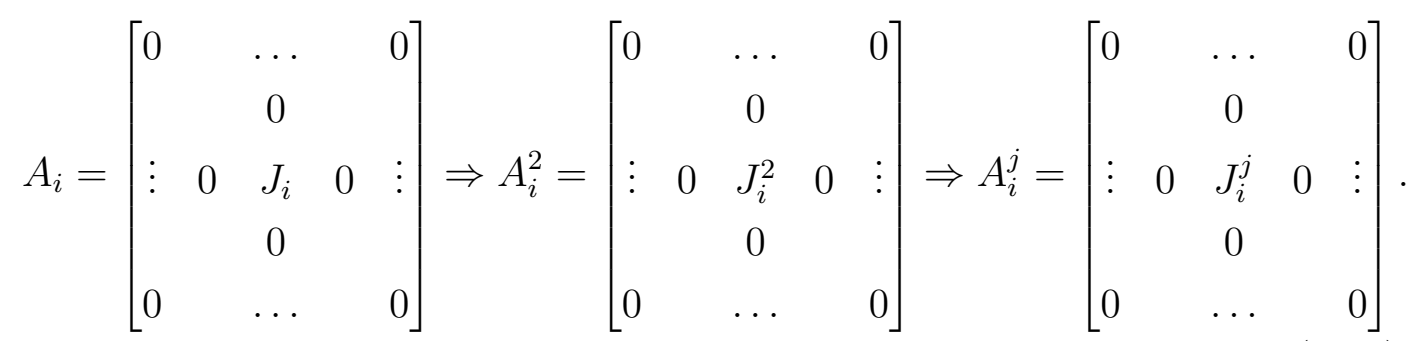

Pela Definição 3.14 temos que para $i=1 \ldots k$

$$
e^{t A_{i}}=\sum_{j=0}^{\infty} \frac{\left(t A_{i}\right)^{j}}{j !}=I+t A_{i}+\frac{t^{2} A_{i}^{2}}{2 !}+\frac{t^{3} A_{i}^{3}}{3 !}+\ldots
$$

De (3-92) e (3-93), usando o resultado da Proposição 3.21 temos

$$
e^{t A_{i}}=\left[\begin{array}{ccc}
I & 0 & 0 \\
0 & e^{t J_{i}} & 0 \\
0 & 0 & I
\end{array}\right] .
$$

Como $A_{i}$ e $A_{l}$ comutam para todo $i \neq l$ então pela Proposição 3.17

$$
\begin{gathered}
e^{t A}=e^{\left(\sum_{i=1}^{k} t A_{i}\right)}=e^{t A_{1}} e^{t A_{2}} \ldots e^{t A_{k}} \\
e^{t A_{1}} e^{t A_{2}}=\left[\begin{array}{ccc}
e^{t J_{1}} & 0 & 0 \\
0 & I & 0 \\
0 & 0 & I
\end{array}\right]\left[\begin{array}{ccc}
I & 0 & 0 \\
0 & e^{t J_{2}} & 0 \\
0 & 0 & I
\end{array}\right]=\left[\begin{array}{ccc}
e^{t J_{1}} & 0 & 0 \\
0 & e^{t J_{2}} & 0 \\
0 & 0 & I
\end{array}\right] \\
\left(e^{t A_{1}} e^{t A_{2}}\right) e^{t A_{3}}=\left[\begin{array}{cccc}
e^{t J_{1}} & 0 & 0 & 0 \\
0 & e^{t J_{2}} & 0 & 0 \\
0 & 0 & I & 0 \\
0 & 0 & 0 & I
\end{array}\right]\left[\begin{array}{cccc}
I & 0 & 0 & 0 \\
0 & I & 0 & 0 \\
0 & 0 & e^{t J_{3}} & 0 \\
0 & 0 & 0 & I
\end{array}\right]=\left[\begin{array}{cccc}
e^{t J_{1}} & 0 & 0 & 0 \\
0 & e^{t J_{2}} & 0 & 0 \\
0 & 0 & e^{t J_{3}} & 0 \\
0 & 0 & 0 & I
\end{array}\right] .
\end{gathered}
$$

Procedendo assim, indutivamente, temos o resultado procurado. 


\section{Proposição 3.23 (Cálculo da Exponencial Matricial)}

Pelo Teorema de Jordan B.63, seja $S$ a matriz inversivel que transforma uma matriz A na forma normal de Jordan $B$ tal que $B=S^{-1} A S$ então

$$
e^{t B}=\left[\begin{array}{ccc}
e^{t J_{1}} & & 0 \\
& \ddots & \\
& & e^{t J_{k}}
\end{array}\right] \text { e } e^{t J_{i}}=e^{\lambda_{i} t}\left[\begin{array}{cccccc}
1 & t & \frac{t^{2}}{2 !} & \frac{t^{3}}{3 !} & \ldots & \frac{t^{n_{i}-1}}{\left(n_{i}-1\right) !} \\
0 & 1 & t & \frac{t^{2}}{2 !} & \ddots & \vdots \\
0 & 0 & 1 & t & \ddots & \frac{t^{3}}{3 !} \\
0 & 0 & 0 & 1 & \ddots & \frac{t^{2}}{2 !} \\
\vdots & \vdots & \vdots & \ddots & \ddots & t \\
0 & 0 & 0 & 0 & \cdots & 1
\end{array}\right] .
$$

Demonstração.

Como $A=S B S^{-1}$ então o resultado vem diretamente da aplicação das Proposições 3.22 e 3.19 sendo, portanto, uma forma de calcular $e^{t A}$ para qualquer matriz $A$.

\section{5}

\section{Solução de Sistemas Lineares com Matriz constante}

Considere o sistema (3-1) em que $A(t)=A$ (matriz real constante) para todo $t \in I$ :

$$
\left\{\begin{array}{l}
x^{\prime}(t)=A x(t)+b(t) \\
x\left(t_{0}\right)=x_{0}
\end{array} \quad \forall t \in I .\right.
$$

Teorema 3.24 Se $\varphi(t)=e^{t A}$ então $\varphi$ é matriz fundamental de $x^{\prime}=A x$.

Demonstração.

Pelo Teorema $3.15, \varphi^{\prime}(t)=A \varphi(t)$ tal que $A=\left(a_{i}^{j}\right)_{i, j=1}^{n}$ e para toda coluna $j \leq n$ temos

$$
\left(\varphi_{i}^{j}(t)\right)^{\prime}=\sum_{k=1}^{n} a_{i}^{k} \varphi_{k}^{j}(t) \Rightarrow\left(\varphi^{j}(t)\right)^{\prime}=A \varphi^{j}(t) .
$$

Assim, $\varphi^{j}$ é solução do sistema $x^{\prime}=A x$ para $j=1 \ldots n$.

Calculando o Wronskiano (Definição 3.7) para o ponto $t_{0}=0$ temos

$$
W(0)=\operatorname{det}(\varphi(0))=|\varphi(0)|=\left|e^{0}\right|=|I|=1 \neq 0 .
$$

Como existe $t_{0}$ tal que $W\left(t_{0}\right) \neq 0$ então, pelo Teorema 3.10 , $\varphi$ é matriz fundamental. 
Teorema 3.25 A solução do sistema (3-98) é dada por

$$
x(t)=e^{\left(t-t_{0}\right) A} x_{0}+\int_{t_{0}}^{t} e^{(t-s) A} b(s) d s .
$$

Demonstração.

Pelo Teorema 3.24 temos que $\varphi(t)=e^{t A}$ é matriz fundamental do sistema.

Pela Proposição 3.18 temos $\varphi^{-1}(t)=e^{-t A}$.

Usando o Teorema 3.13 considerando $A(t)=A$ (constante) temos

$$
\begin{aligned}
& x(t)=\varphi(t)\left(\varphi^{-1}\left(t_{0}\right) x_{0}+\int_{t_{0}}^{t} \varphi^{-1}(s) b(s) d s\right) \\
& x(t)=e^{t A}\left(e^{-t_{0} A} x_{0}+\int_{t_{0}}^{t} e^{-s A} b(s) d s\right) .
\end{aligned}
$$

Como $(t A),\left(-t_{0} A\right)$ e $(-s A)$ comutam entre si, temos pela Proposição 3.17

$$
x(t)=e^{\left(t-t_{0}\right) A} x_{0}+\int_{t_{0}}^{t} e^{(t-s) A} b(s) d s .
$$




\section{4}

\section{Estabilidade de Soluções de Sistemas de EDO}

Seja uma função $F: D \subset \mathbb{R}^{1+n} \rightarrow \mathbb{R}^{n}$ e o sistema de equações diferenciais ordinárias, definido para $t \geq t_{0} \in \mathbb{R}$

$$
x^{\prime}=F(t, x) .
$$

\section{Definição 4.1 (Estabilidade de Soluções) [9]}

Uma solução $\psi$ de (4-1) é dita estável se para todo $\varepsilon>0$ existir um $\delta>0$ tal que para toda solução $\varphi$ do sistema em que $\left\|\varphi\left(t_{0}\right)-\psi\left(t_{0}\right)\right\|<\delta$ tivermos $\|\varphi(t)-\psi(t)\|<\varepsilon$ para todo $t \geq t_{0}$. Caso contrário, dizemos que $\psi$ é instável.

Nas seções a seguir, consideraremos $t_{0}=0$ e $F(t, 0)=0 \in \mathbb{R}^{n}$ para todo $t \geq 0$ e decomporemos $F$ na soma de uma função não-linear $f: D \rightarrow \mathbb{R}^{n} \mathrm{com}$ uma função linear $A x$ em que $A$ é uma matriz real $(n \times n)$ constante, ou seja, consideraremos o seguinte sistema não-linear de EDO, definido para todo $t \geq 0$

$$
x^{\prime}=A x+f(t, x) .
$$

Na seção 4.3, veremos um exemplo de como podemos fazer esta decomposição da função $F$.

\section{Definição 4.2 (Solução Estacionária)}

As soluções constantes de um sistema de equações diferenciais ordinárias são denominadas soluções estacionárias do sistema.

Notemos que, como $F(t, 0)=f(t, 0)=0 \in \mathbb{R}^{n}$ para todo $t \geq 0$ então a função identicamente nula é uma solução estacionária destes sistemas. Analisaremos neste capítulo a estabilidade desta solução.

Iniciaremos demonstrando um importante resultado que nos permite limitar o valor da norma de uma função exponencial matricial por uma função exponencial real. Este resultado será utilizado ao longo deste capítulo. 
Proposição 4.3 Seja A uma matriz $(n \times n)$ tal que as partes reais de seus autovalores sejam menores do que $\beta \in \mathbb{R}$ então existe $\alpha \geq 1$ tal que

$$
\left\|e^{t A}\right\| \leq \alpha e^{\beta t} \text { para todo } t \geq 0
$$

Demonstração.

Vimos na Proposição 3.23 que existem matrizes $S$ e $B(n \times n)$ tais que

$$
e^{t A}=S e^{t B} S^{-1}
$$

em que

$$
e^{t B}:=\left[\begin{array}{ccc}
e^{t J_{1}} & & 0 \\
& \ddots & \\
0 & & e^{t J_{k}}
\end{array}\right] \text { e } e^{t J_{l}}=e^{\lambda_{l} t}\left[\begin{array}{cccccc}
1 & t & \frac{t^{2}}{2 !} & \frac{t^{3}}{3 !} & \ldots & \frac{t^{n_{l}-1}}{\left(n_{l}-1\right) !} \\
0 & 1 & t & \frac{t^{2}}{2 !} & \ddots & \vdots \\
0 & 0 & 1 & t & \ddots & \frac{t^{3}}{3 !} \\
0 & 0 & 0 & 1 & \ddots & \frac{t^{2}}{2 !} \\
\vdots & \vdots & \vdots & \ddots & \ddots & t \\
0 & 0 & 0 & 0 & \ldots & 1
\end{array}\right]
$$

tal que

$$
\sum_{l=1}^{k} n_{l}=n \Rightarrow n_{l} \leq n \quad l=1 \ldots k .
$$

Seja

$$
\left(c_{i j}(t)\right)_{i, j=1}^{n}:=e^{t B}
$$

Usando as propriedades da norma matricial induzida (Definição B.10) temos

$$
\left\|e^{t A}\right\| \leq\|S\|\left\|e^{t B}\right\|\left\|S^{-1}\right\|=: \alpha_{1}\left\|e^{t B}\right\|
$$

em que

$$
\alpha_{1}:=\|S\|\left\|S^{-1}\right\| \geq\left\|S S^{-1}\right\|=\|I\| \Rightarrow \alpha_{1} \geq 1 .
$$

Seja $\lambda=\max \left\{\operatorname{Re} \lambda_{1}, \ldots, \operatorname{Re} \lambda_{k}\right\}$ então $\lambda<\beta$ e para todo $l \leq k$ e $t \geq 0$

$$
\lambda_{l}=: a_{l}+i b_{l} \Rightarrow\left|e^{\lambda_{l}}\right| \leq\left|e^{a_{l}}\right|\left|e^{i b_{l}}\right| \leq e^{\lambda} .1=e^{\lambda} \Rightarrow\left|e^{\lambda_{l} t}\right| \leq e^{\lambda t} .
$$

Então, para todo $i, j \leq n$ existe $n_{i j}$ tal que $1 \leq n_{i j} \leq n$

$$
\left|c_{i j}(t)\right| \leq \frac{t^{n_{i j}-1} e^{\lambda t}}{\left(n_{i j}-1\right) !} .
$$

Assim, se

$$
\begin{aligned}
0 \leq t \leq 1 & \Rightarrow\left|c_{i j}(t)\right| \leq e^{\lambda t} \leq e^{\beta t} \\
t \geq 1 & \Rightarrow\left|c_{i j}(t)\right| \leq t^{n_{i j}-1} e^{\lambda t} \leq t^{n-1} e^{\lambda t}=\frac{t^{n-1}}{e^{(\beta-\lambda) t}} e^{\beta t} \leq \frac{n !}{(\beta-\lambda)^{n}} e^{\beta t}
\end{aligned}
$$

sendo a última desigualdade decorrente de que se $t \geq 1$ e $\delta:=(\beta-\lambda)>0$

$$
\frac{\delta^{n} t^{n-1}}{n !}=\frac{(\delta t)^{n}}{t . n !} \leq \frac{(\delta t)^{n}}{n !} \leq \sum_{m=0}^{\infty} \frac{(\delta t)^{m}}{m !}=e^{\delta t}
$$


De (4-11), escolhendo $\alpha_{2}:=\max \left\{1, \frac{n !}{(\beta-\lambda)^{n}}\right\}$ temos

$$
\left\|e^{t B}\right\|_{1}=\max _{j} \sum_{i=1}^{n}\left|c_{i j}(t)\right| \leq n \alpha_{2} e^{\beta t} \quad \forall t \geq 0
$$

De (4-7) e (4-13), definindo $\alpha:=n \alpha_{1} \alpha_{2}>0$ temos

$$
\left\|e^{t A}\right\|_{1} \leq \alpha_{1}\left\|e^{t B}\right\|_{1} \leq \alpha_{1}\left(n \alpha_{2} e^{\beta t}\right)=\alpha e^{\beta t} \quad \forall t \geq 0 .
$$

Pela equivalência entre as normas matriciais, temos o resultado procurado.

\section{1}

\section{Estabilidade Assintótica de Soluções}

Definição 4.4 [9]

Sejam $\psi$ e $\varphi$ soluções conforme Definição 4.1, dizemos que a solução $\psi$ é assintoticamente estável se, além de ser estável, tivermos também

$$
\lim _{t \rightarrow \infty}\|\varphi(t)-\psi(t)\|=0 .
$$

Visando fixar os conceitos de estabilidade, analisaremos a seguir o caso particular do sistema (4-2) em que $f \equiv 0 \in \mathbb{R}^{n}$, ou seja, um sistema linear homogêneo $x^{\prime}=A x$.

Proposição 4.5 Seja o sistema (4-2) com $f \equiv 0 \in \mathbb{R}^{n}$. Se todos os autovalores de A possuírem partes reais negativas então a solução identicamente nula do sistema $x^{\prime}=A x$ é assintoticamente estável.

Demonstração.

Seja $\varphi$ uma solução qualquer do sistema então pelo Teorema 3.25 temos que

$$
\varphi(t)=e^{t A} \varphi(0)
$$

Sejam $\lambda_{1}, \ldots, \lambda_{k}$ os autovalores de $A$, definimos $\lambda=\max _{i \leq k} \operatorname{Re} \lambda_{i}$ e como $\lambda<0$ temos que existe $\beta=\frac{\lambda}{2}<0$ tal que para todo $i \leq k$ temos $\lambda_{i} \leq \lambda<\beta<0$ e logo, pela Proposição 4.3, existe $\alpha \geq 1$ tal que

$$
\left\|e^{t A}\right\| \leq \alpha e^{\beta t} \quad \forall t \geq 0
$$

De (4-15) e (4-16) temos

$$
\|\varphi(t)\| \leq \alpha e^{\beta t}\|\varphi(0)\| \quad \forall t \geq 0 .
$$

Como $\beta<0$ temos que $e^{\beta t} \leq 1$ e logo

$$
\|\varphi(t)\| \leq \alpha\|\varphi(0)\| \quad \forall t \geq 0 .
$$


Seja $\varepsilon>0$ escolhendo $\delta=\frac{\varepsilon}{\alpha}>0$ temos que

$$
\|\varphi(0)\|<\delta \Rightarrow\|\varphi(t)\| \leq \alpha\|\varphi(0)\|<\alpha \frac{\varepsilon}{\alpha}=\varepsilon \quad \forall t \geq 0 .
$$

Assim, seja $\psi$ a solução identicamente nula $(\psi(t)=0$ para todo $t \geq 0)$, temos que para todo $\varepsilon>0$ existe $\delta>0$ tal que $\|\varphi(0)-\psi(0)\|<\delta$ implica que $\|\varphi(t)-\psi(t)\|<\varepsilon$ para todo $t \geq 0$, logo $\psi$ é estável pela Definição 4.1 .

De (4-17), como $\beta<0$ temos

$$
\lim _{t \rightarrow \infty}\|\varphi(t)-\psi(t)\|=\lim _{t \rightarrow \infty}\|\varphi(t)\|=0
$$

Desta forma, pela Definição 4.4, a solução $\psi$ (identicamente nula) é assintoticamente estável.

A seguir, analisaremos o comportamento da solução identicamente nula de um sistema não linear que se aproxima de um sistema linear para um $x$ muito próximo a origem. Mais formalmente, veremos o caso em que

$$
\lim _{x \rightarrow 0} \frac{\|f(t, x)\|}{\|x\|}=0 \text { uniformemente em } t \text { para } t \geq 0
$$

ou seja, para todo $\varepsilon>0$ existe $\delta>0$ tal que $\|f(t, x)\| \leq \varepsilon\|x\|$ para todo $t \geq 0$ e $\|x\|<\delta$.

Teorema 4.6 Seja o sistema (4-2) em que todos os autovalores de A possuem partes reais negativas. Suponha existir $\delta_{0}>0$ em que $f$ é contínua para todo $t \geq 0$ e $\|x\|<\delta_{0}$. Se para todo $\varepsilon>0$ existir $\delta_{1}>0$ tal que $\|f(t, x)\| \leq \varepsilon\|x\|$ para todo $t \geq 0 e\|x\|<\delta_{1}$ então a solução identicamente nula é assintoticamente estável.

Demonstração.

Conforme vimos na Proposição 4.5, existem $\beta<0$ e $\alpha \geq 1$ tais que

$$
\left\|e^{t A}\right\| \leq \alpha e^{\beta t} \text { para todo } t \geq 0
$$

Logo, para todo $0 \leq s \leq t$ temos também que

$$
\left\|e^{(t-s) A}\right\| \leq \alpha e^{\beta(t-s)} .
$$


Seja $\varphi$ uma solução qualquer do sistema, considerando $b(t):=f(t, \varphi(t))$ então, se $\|\varphi(s)\|<\delta_{0}$ para todo $s \in[0, t], b(t)$ é contínua e logo podemos usar o Teorema 3.25 e temos

$$
\varphi(t)=e^{t A} \varphi(0)+\int_{0}^{t} e^{(t-s) A} f(s, \varphi(s)) d s .
$$

Usando as desigualdades das normas matriciais e vetoriais, de (4-21) e (4-22) temos que se $\|\varphi(s)\|<\delta_{0}$ para todo $s \in[0, t]$ então

$$
\begin{aligned}
& \|\varphi(t)\| \leq\left\|e^{t A}\right\|\|\varphi(0)\|+\int_{0}^{t}\left\|e^{(t-s) A}\right\|\|f(s, \varphi(s))\| d s \\
& \|\varphi(t)\| \leq \alpha e^{\beta t}\|\varphi(0)\|+\int_{0}^{t} \alpha e^{\beta(t-s)}\|f(s, \varphi(s))\| d s .
\end{aligned}
$$

Seja $\varepsilon>0$, como $\lim _{x \rightarrow 0} \frac{\|f(t, x)\|}{\|x\|}=0$ então, pela Definição de Limite B.24, existe $\delta_{1}>0$ tal que se $\|x\|<\delta_{1}$

$$
\frac{\|f(t, x)\|}{\|x\|}<\frac{\varepsilon}{\alpha} \Rightarrow\|f(t, x)\|<\frac{\varepsilon}{\alpha}\|x\| \quad \forall t \geq 0 .
$$

Assim, de (4-24) e (4-25), se $\|\varphi(s)\|<\delta_{2}:=\min \left\{\delta_{1}, \delta_{0}\right\}$ para todo $s \in[0, t]$

$$
\begin{aligned}
\|\varphi(t)\| & \leq \alpha e^{\beta t}\|\varphi(0)\|+\int_{0}^{t} \alpha e^{\beta(t-s)}\left(\frac{\varepsilon}{\alpha}\|\varphi(s)\|\right) d s \\
e^{-\beta t}\|\varphi(t)\| & \leq \alpha\|\varphi(0)\|+\int_{0}^{t} \varepsilon e^{-\beta s}\|\varphi(s)\| d s .
\end{aligned}
$$

Pela Proposição A.12 considerando

$$
w(t):=e^{-\beta t}\|\varphi(t)\| \quad, \quad y(t):=\alpha\|\varphi(0)\| \quad \text { e } \quad z(t):=\varepsilon
$$

temos de (4-26)

$$
\begin{aligned}
e^{-\beta t}\|\varphi(t)\| & \leq \alpha\|\varphi(0)\|+\int_{0}^{t} \varepsilon \alpha\|\varphi(0)\|\left(e^{\int_{s}^{t} \varepsilon d u}\right) d s \\
& \leq \alpha\|\varphi(0)\|\left(1+\varepsilon \int_{0}^{t} e^{\varepsilon(t-s)} d s\right) \\
& =\alpha\|\varphi(0)\|\left(1-\left.e^{\varepsilon(t-s)}\right|_{0} ^{t}\right) \\
& =\alpha\|\varphi(0)\| e^{\varepsilon t} .
\end{aligned}
$$

Logo

$$
\|\varphi(t)\| \leq \alpha\|\varphi(0)\| e^{(\beta+\varepsilon) t}
$$

Se $\varepsilon<-\beta$ então $\eta:=\beta+\varepsilon<0$ e temos que se $\|\varphi(s)\|<\delta_{2}$ para todo $s \in[0, t]$

$$
\|\varphi(t)\| \leq \alpha\|\varphi(0)\| e^{\eta t} \quad \forall t \geq 0
$$


Afirmação: $S e\|\varphi(0)\|<\frac{\delta_{2}}{\alpha} \leq \delta_{2}$ entã $\|\varphi \varphi(t)\|<\delta_{2}$ para todo $t \geq 0$.

Pela continuidade de $\varphi$ em 0 , seja $\varepsilon^{\prime}=\delta_{2}-\|\varphi(0)\|>0$ existe $t_{0}>0$ tal que se $|s|<t_{0}$ então $\|\varphi(s)-\varphi(0)\|<\varepsilon^{\prime}$ e $\operatorname{logo}\|\varphi(s)\|<\delta_{2}$ para todo $s \in\left(0, t_{0}\right)$.

Sejam

$$
P:=\left\{t>0:\|\varphi(s)\|<\delta_{2} \forall s \in(0, t)\right\} \quad \text { e } T:=\sup P
$$

então $T \geq t_{0}>0$. Suponha por absurdo que $T<\infty$, ou seja, $T \in \mathbb{R}$.

Como $\|\varphi(s)\|<\delta_{2}$ para todo $s \in(0, T)$ então, de (4-30)

$$
\|\varphi(T)\| \leq \alpha\|\varphi(0)\| e^{\eta T} \leq \alpha\|\varphi(0)\|<\delta_{2}
$$

Mas, pela continuidade de $\varphi$ em $T$, existe $t_{1}>0$ tal que $\|\varphi(t)\|<\delta_{2}$ para todo $t \in\left(T, T+t_{1}\right)$. Absurdo, pois $T=\sup P \geq T+t_{1}>T$.

Assim, escolhendo $\delta:=\min \left\{\frac{\varepsilon}{\alpha}, \frac{\delta_{2}}{\alpha}\right\}$ temos $\|\varphi(0)\|<\delta$ e $\|\varphi(t)\|<\delta_{2}$ para todo $t \geq 0$ e, portanto, podemos usar (4-30)

$$
\|\varphi(t)\| \leq \alpha\|\varphi(0)\| e^{\eta t} \leq \alpha\|\varphi(0)\|<\varepsilon \quad \forall t \geq 0 .
$$

Se $\varepsilon \geq-\beta$, escolhemos qualquer $\delta$ obtido para algum $\varepsilon_{0}$ (fixo) tal que $\varepsilon_{0}<-\beta$ e temos por (4-33) que se $\|\varphi(0)\|<\delta$ e $\eta:=\beta+\varepsilon_{0}<0$ então

$$
\|\varphi(t)\| \leq \alpha\|\varphi(0)\| e^{\eta t} \leq \alpha\|\varphi(0)\|<\varepsilon_{0}<\varepsilon \quad \forall t \geq 0 .
$$

De (4-33) e (4-34), para todo $\varepsilon>0$ existe $\delta>0$ tal que se $\|\varphi(0)\|<\delta$ então

$$
\|\varphi(t)\|<\varepsilon \quad \forall t \geq 0 .
$$

Logo, pela Definição 4.1, temos que a solução identicamente nula é estável. Ainda, como $\eta<0$ temos $\lim _{t \rightarrow \infty} e^{\eta t}=0$ e portanto

$$
\lim _{t \rightarrow \infty}\|\varphi(t)\|=0
$$

Desta forma, pela Definição 4.4, temos que a solução identicamente nula é assintoticamente estável.

Em seguida, enfraqueceremos um pouco as hipóteses do teorema anterior e analisaremos um sistema não linear que se aproxima de um sistema linear próximo a origem a partir de um instante $t$ suficientemente grande. 
Teorema 4.7 Seja o sistema (4-2) em que todos autovalores de A possuem partes reais negativas. Suponha existirem $\delta_{0}>0$ e $K>0$ tais que para todo $t \geq 0$ e $\|x\|<\delta_{0}$ tem-se que $f$ é contínua e $\|f(t, x)\| \leq K\|x\|$.

Se para todo $\varepsilon>0$ existirem $\delta_{1}>0$ e $T \geq 0$ tais que $\|f(t, x)\| \leq \varepsilon\|x\|$ para todo $t \geq T e\|x\|<\delta_{1}$ então a solução identicamente nula é assintoticamente estável.

Demonstração.

Seja $\varphi$ uma solução (não identicamente nula) então

$$
\varphi^{\prime}(t)=A \varphi(t)+f(t, \varphi(t)) .
$$

Se $\|\varphi(t)\|<\delta_{0}$ então $b(t):=f(t, \varphi(t))$ é contínua e, pelo Teorema de Existência e Unicidade 3.1 , temos que $\varphi(t) \neq 0$ para todo $t \geq 0$ (caso contrário $\varphi$ teria que ser a solução identicamente nula).

Como $\|\varphi(t)\| \neq 0$, pela regra da cadeia (Teorema B.38) e do produto (Teorema B.39) das derivadas temos, respectivamente

$$
\left(\|\varphi\|^{2}\right)^{\prime}=2\|\varphi\|\|\varphi\|^{\prime} \quad \text { e } \quad\left(\|\varphi\|^{2}\right)^{\prime}=(\varphi \cdot \varphi)^{\prime}=2 \varphi \cdot \varphi^{\prime} .
$$

Logo

e com isto

$$
\|\varphi\|^{\prime}=\frac{\varphi}{\|\varphi\|} \cdot \varphi^{\prime} \leq\left\|\frac{\varphi}{\|\varphi\|}\right\|\left\|\varphi^{\prime}\right\|=1 .\left\|\varphi^{\prime}\right\|
$$

$$
\|\varphi\|^{\prime} \leq\left\|\varphi^{\prime}\right\| \text {. }
$$

Assim, se $\|\varphi(t)\|<\delta_{0}$, usando as desigualdades das normas vetoriais e matriciais induzidas (Definição B.10) temos

$$
\begin{aligned}
\|\varphi(t)\|^{\prime} \leq\left\|\varphi^{\prime}(t)\right\| & \leq\|A\|\|\varphi(t)\|+\|f(t, \varphi(t))\| \\
& \leq\|\varphi(t)\|(\|A\|+K) .
\end{aligned}
$$

Seja $\alpha:=(\|A\|+K)>0(\alpha \in \mathbb{R})$ então

$$
\frac{\|\varphi(t)\|^{\prime}}{\|\varphi(t)\|} \leq \alpha \Rightarrow \int_{0}^{t} \frac{\|\varphi(t)\|^{\prime}}{\|\varphi(t)\|} d s \leq \int_{0}^{t} \alpha d s .
$$

Assim, temos

$$
\ln \left(\frac{\|\varphi(t)\|}{\|\varphi(0)\|}\right) \leq \alpha t \Rightarrow\|\varphi(t)\| \leq\|\varphi(0)\| e^{\alpha t}
$$

Seja $\varepsilon>0$ então existem $\delta_{1}>0$ e $T \geq 0$ tais que $\|f(t, x)\| \leq \varepsilon\|x\|$ para todo $t \geq T$ e $\|x\|<\delta_{1}$. 
Definimos:

$$
s:=t-T \quad, \quad g(s, x):=f(s+T, x) \quad e \quad \phi(s):=\varphi(s+T)
$$

e temos para todo $s \geq 0$ e $\|x\|<\delta_{1}$

$$
\|g(s, x)\|=\|f(s+T, x)\|<\varepsilon\|x\| .
$$

Logo

$$
\lim _{x \rightarrow 0} \frac{\|g(s, x)\|}{\|x\|}=0 .
$$

Pelo Teorema 4.6, existe $\delta_{2}>0$ tal que se $\|\phi(0)\|<\delta_{2}$ temos

$$
\|\phi(s)\|<\varepsilon \quad \forall s \geq 0 \Rightarrow\|\varphi(t)\|<\varepsilon \quad \forall t \geq T
$$

o que pela definição de limite nos fornece

$$
\lim _{s \rightarrow \infty}\|\phi(s)\|=0 \Rightarrow \lim _{t \rightarrow \infty}\|\varphi(t)\|=0
$$

Escolhendo $\delta:=\frac{\min \left\{\delta_{2}, \delta_{1}, \varepsilon\right\}}{e^{\alpha T}}$, de (4-42) temos que se $\|\varphi(0)\|<\delta$

$$
\|\phi(0)\|=\|\varphi(T)\| \leq\|\varphi(0)\| e^{\alpha T}<\delta_{2}
$$

e para todo $t \leq T$ temos ainda

$$
\|\varphi(t)\| \leq\|\varphi(0)\| e^{\alpha t} \leq\|\varphi(0)\| e^{\alpha T}<\varepsilon
$$

De (4-48), como $\|\phi(0)\|<\delta_{2}$ então são válidas as equações (4-46) e (4-47).

Assim, para todo $\varepsilon>0$ existe $\delta>0$ tal que $\|\varphi(0)\|<\delta$ implica que

$$
\|\varphi(t)\|<\varepsilon \quad \forall t \geq 0 \quad \text { e } \quad \lim _{t \rightarrow \infty}\|\varphi(t)\|=0
$$

Pela Definição 4.4, temos que a solução identicamente nula é assintoticamente estável.

\section{2}

\section{Instabilidade de Soluções}

Nesta seção, veremos o caso em que a matriz $A$ possui ao menos um autovalor com parte real positiva e concluiremos que a solução identicamente nula é instável, alterando apenas esta hipótese no teorema anterior. 
Teorema 4.8 Seja o sistema (4-2) em que ao menos um autovalor de A possui parte real positiva. Suponha existirem $\delta_{0}>0$ e $K>0$ tais que para todo $t \geq 0$ $e\|x\|<\delta_{0}$ tem-se que $f$ é contínua e $\|f(t, x)\| \leq K\|x\|$.

Se para todo $\varepsilon>0$ existirem $\delta_{1}>0$ e $T \geq 0$ tais que $\|f(t, x)\| \leq \varepsilon\|x\|$ para todo $t \geq T$ e $\|x\|<\delta_{1}$ então a solução identicamente nula é instável.

Demonstração.

Pelo Teorema de Jordan B.63, existe matriz inversível $S$ e matriz na forma normal de Jordan $B$ tais que

$$
A=S B S^{-1}
$$

em que $\lambda_{1}, \ldots, \lambda_{n}$ são os elementos da diagonal de $B$, ou seja, são os autovalores de $A$. Sem perda de generalidade, suponha $\operatorname{Re}\left(\lambda_{1}\right) \geq \operatorname{Re}\left(\lambda_{2}\right) \geq \ldots \operatorname{Re}\left(\lambda_{n}\right)$.

Sejam as matrizes $B^{+}$e $B^{-}$tais que

$$
B=:\left[\begin{array}{cc}
B^{+} & 0 \\
0 & B^{-}
\end{array}\right]
$$

em que $B^{+}$é um bloco $(k \times k)$ que contem na diagonal os autovalores com parte real positiva e $B^{-}$um bloco $(n-k \times n-k)$ contendo os demais autovalores com parte real não positiva.

Assim, existe $\beta>0$ tal que para todo elemento $\lambda_{i}$ da diagonal de $B^{+}$ temos $\operatorname{Re} \lambda_{i}>\beta$.

Seja $\alpha=\frac{\beta}{4}$, escolhendo adequadamente $S$ podemos obter uma matriz $B$ cuja diagonal contém os autovalores de $A$ (ordenados pela parte real de seus autovalores) e acima dela elementos $\alpha_{i} \in\{0, \alpha\}$ para todo $i=1 \ldots(n-1)$, com todos demais elementos nulos.

De (4-51)

$$
\begin{aligned}
x^{\prime}=A x+f(t, x) \Rightarrow x^{\prime} & =S B S^{-1} x+f(t, x) \\
S^{-1} x^{\prime} & =S^{-1} S B S^{-1} x+S^{-1} f(t, x) \\
S^{-1} x^{\prime} & =B S^{-1} x+S^{-1} f(t, x) .
\end{aligned}
$$

Seja a mudança de variável $y=S^{-1} x$ e $g(t, y)=S^{-1} f(t, x)=S^{-1} f(t, S y)$.

Temos que o sistema de EDO em $y$ é dado por

$$
y^{\prime}=B y+g(t, y) \text {. }
$$


Note que o sistema está bem definido pois como $S$ é inversível e $y=S^{-1} x$ é uma aplicação linear bijetora então, mesmo que $y$ seja complexo, $x=S y$ será real pois $S B S^{-1}=A$ é uma matriz real e logo $x$ é solução de um sistema real. Assim, temos que $\psi$ é solução do sistema de (4-2), se e somente se, $\varphi:=S^{-1} \psi$ é solução de (4-54).

Desta forma, seja $\varphi$ uma solução de (4-54), definimos:

$$
\begin{aligned}
& {\left[\begin{array}{l}
\varphi^{+} \\
\varphi^{-}
\end{array}\right]^{\prime}=\left[\begin{array}{cc}
B^{+} & 0 \\
0 & B^{-}
\end{array}\right]\left[\begin{array}{l}
\varphi^{+} \\
\varphi^{-}
\end{array}\right]+\left[\begin{array}{l}
g^{+} \\
g^{-}
\end{array}\right] \text {em que }} \\
& \varphi^{+}=\left(\varphi_{1}, \ldots, \varphi_{k}\right) \quad e \quad \varphi^{-}=\left(\varphi_{k+1}, \ldots, \varphi_{n}\right) \\
& g^{+}=\left(g_{1}, \ldots, g_{k}\right) \quad \text { e } g^{-}=\left(g_{k+1}, \ldots, g_{n}\right) \text {. }
\end{aligned}
$$

em que para todo $i=1 \ldots n, \varphi_{i}$ e $g_{i}$ são as funções coordenadas de $\varphi$ e $g$.

Seja $0<\varepsilon<\frac{\beta}{2}$ então existem $\delta_{1}>0$ e $T \geq 0$ tais que se $\|y\|<\delta_{1}$

$$
\|g(t, y)\| \leq \varepsilon\|y\| \quad \forall t \geq T .
$$

Suponha por absurdo que a solução identicamente nula de $(4-54)$ é estável. Com isto, pela Definição 4.1, para todo $\varepsilon_{1}>0$ existe $\delta>0$ tal que se:

$$
\|\varphi(T)\|^{2}=\left\|\varphi^{+}(T)\right\|^{2}+\left\|\varphi^{-}(T)\right\|^{2}<\delta^{2}
$$

então

$$
\|\varphi(t)\|^{2}=\left\|\varphi^{+}(t)\right\|^{2}+\left\|\varphi^{-}(t)\right\|^{2}<\varepsilon_{1}^{2}:=\delta_{1}^{2} \quad \forall t \geq T .
$$

Escolhemos $\varphi$ tal que $\|\varphi(T)\|<\delta$ e

$$
\left\|\varphi^{+}(T)\right\|=2\left\|\varphi^{-}(T)\right\| .
$$

Como

$$
\left(\left\|\varphi^{+}\right\|^{2}\right)^{\prime}=2\left\|\varphi^{+}\right\|\left\|\varphi^{+}\right\|^{\prime}=\left(\sum_{i=1}^{k}\left|\varphi_{i}\right|^{2}\right)^{\prime}=\left(\sum_{i=1}^{k} \varphi_{i} \overline{\varphi_{i}}\right)^{\prime}=\sum_{i=1}^{k}\left(\varphi_{i}^{\prime} \overline{\varphi_{i}}+\varphi_{i} \bar{\varphi}_{i}^{\prime}\right) .
$$

De $(4-55)$

$$
2\left\|\varphi^{+}\right\|\left\|\varphi^{+}\right\|^{\prime}=\sum_{i=1}^{k}\left(\left(B_{i}^{+} \varphi^{+}+g_{i}\right) \overline{\varphi_{i}}+\overline{\left(B_{i}^{+} \varphi^{+}+g_{i}\right)} \varphi_{i}\right)
$$

em que $B_{i}^{+}$é a i-ésima linha de $B^{+}$, ou seja, $B_{i}^{+}=\left(0, \ldots, \lambda_{i}, \alpha_{i}, 0, \ldots, 0\right)$ em que $\lambda_{i}$ está na i-ésima coluna e $\alpha_{i} \in\{0, \alpha\}$. 
Como

$$
B_{i}^{+} \varphi^{+} \overline{\varphi_{i}}=\left(\lambda_{i} \varphi_{i}+\alpha_{i} \varphi_{i+1}\right) \overline{\varphi_{i}}
$$

então

$$
\begin{aligned}
B_{i}^{+} \varphi^{+} \overline{\varphi_{i}}+\overline{B_{i}^{+} \varphi^{+}} \varphi_{i} & =\left(\lambda_{i}+\overline{\lambda_{i}}\right) \varphi_{i} \overline{\varphi_{i}}+\alpha_{i}\left(\varphi_{i+1} \overline{\varphi_{i}}+\overline{\varphi_{i+1}} \varphi_{i}\right) \\
& =2 \operatorname{Re}\left(\lambda_{i}\right)\left|\varphi_{i}\right|^{2}+\alpha_{i}\left(\varphi_{i+1} \overline{\varphi_{i}}+\overline{\varphi_{i+1}} \varphi_{i}\right) .
\end{aligned}
$$

Sejam $v:=\left(\varphi_{2}, \ldots, \varphi_{k}\right)$ e $w:=\left(\varphi_{1}, \ldots, \varphi_{k-1}\right)$, temos

$$
\left|\sum_{i=1}^{k-1} \alpha_{i} \overline{\varphi_{i+1}} \varphi_{i}\right|<\alpha\left|\sum_{i=1}^{k-1} \overline{\varphi_{i+1}} \varphi_{i}\right|=\alpha|v . w|<\alpha\|v\|\|w\|<\alpha\left\|\varphi^{+}\right\|\left\|\varphi^{+}\right\|=\alpha\left\|\varphi^{+}\right\|^{2}
$$

De (4-63), usando (4-64) e considerando que $\operatorname{Re}\left(\lambda_{i}\right)>\beta$ temos

$$
\sum_{i=1}^{k}\left(B_{i}^{+} \varphi^{+} \overline{\varphi_{i}}+\overline{B_{i}^{+} \varphi^{+}} \varphi_{i}\right) \geq 2(\beta-\alpha)\left\|\varphi^{+}\right\|^{2}
$$

Ainda,

$$
\sum_{i=1}^{k} g_{i} \overline{\varphi_{i}}+\overline{g_{i}} \varphi_{i}=g^{+} \overline{\varphi^{+}}+\overline{g^{+}} \varphi^{+} \leq 2\left\|g^{+}\right\|\left\|\varphi^{+}\right\| \leq 2\|g\|\left\|\varphi^{+}\right\| .
$$

De (4-61), (4-65) e (4-66)

$$
\left\|\varphi^{+}\right\|^{\prime} \geq(\beta-\alpha)\left\|\varphi^{+}\right\|-\left\|g^{+}\right\| .
$$

De $(4-56)$

$$
\left\|\varphi^{+}\right\|^{\prime} \geq(\beta-\alpha)\left\|\varphi^{+}\right\|-\varepsilon\|\varphi\| .
$$

Como $\varepsilon<\frac{\beta}{2}$ e $\alpha=\frac{\beta}{4}$

$$
\left\|\varphi^{+}\right\|^{\prime} \geq \frac{3 \beta}{4}\left\|\varphi^{+}\right\|-\varepsilon\left(\left\|\varphi^{+}\right\|+\left\|\varphi^{-}\right\|\right) \geq \frac{\beta}{4}\left\|\varphi^{+}\right\|-\varepsilon\left\|\varphi^{-}\right\| .
$$

Procedendo analogamente para $\varphi^{-}$temos

$$
\begin{aligned}
B_{i}^{-} \varphi^{-} \overline{\varphi_{i}}+\overline{B_{i}^{-} \varphi^{-}} \varphi_{i} & =\left(\lambda_{i}+\overline{\lambda_{i}}\right) \varphi_{i} \overline{\varphi_{i}}+\alpha_{i}\left(\overline{\varphi_{i+1}} \varphi_{i}+\varphi_{i+1} \overline{\varphi_{i}}\right) \\
\leq & 0+\alpha\left(\overline{\varphi_{i+1}} \varphi_{i}+\varphi_{i+1} \overline{\varphi_{i}}\right) .
\end{aligned}
$$

Com isto, temos:

$$
\sum_{i=k+1}^{n}\left(B_{i}^{-} \varphi^{-} \overline{\varphi_{i}}+\overline{B_{i}^{-} \varphi^{-}} \varphi_{i}\right) \leq 2 \alpha\left\|\varphi^{-}\right\|^{2}
$$

e $\log 0$

$$
\left\|\varphi^{-}\right\|^{\prime} \leq \alpha\left\|\varphi^{-}\right\|+\varepsilon\|\varphi\| \leq \frac{\beta}{4}\left\|\varphi^{-}\right\|+\varepsilon\left(\left\|\varphi^{-}\right\|+\left\|\varphi^{+}\right\|\right) .
$$


De (4-69) e (4-72)

$$
\left(\left\|\varphi^{+}\right\|-\left\|\varphi^{-}\right\|\right)^{\prime} \geq \frac{\beta}{4}\left(\left\|\varphi^{+}\right\|-\left\|\varphi^{-}\right\|\right)-\varepsilon\left(\left\|\varphi^{+}\right\|+2\left\|\varphi^{-}\right\|\right) .
$$

Como $\varepsilon$ é arbitrário, quando $\varepsilon \rightarrow 0$ temos

$$
\left(\left\|\varphi^{+}(t)\right\|-\left\|\varphi^{-}(t)\right\|\right)^{\prime} \geq \frac{\beta}{4}\left(\left\|\varphi^{+}(t)\right\|-\left\|\varphi^{-}(t)\right\|\right) .
$$

Integrando de $T$ a $t$ temos

$$
\begin{aligned}
\ln \left(\frac{\left\|\varphi(t)^{+}\right\|-\left\|\varphi^{-}(t)\right\|}{\left\|\varphi^{+}(T)\right\|-\left\|\varphi^{-}(T)\right\|}\right) & \geq \frac{\beta}{4}(t-T) \\
\left\|\varphi(t)^{+}\right\|-\left\|\varphi^{-}(t)\right\| & \geq\left(\left\|\varphi^{+}(T)\right\|-\left\|\varphi^{-}(T)\right\|\right) e^{\frac{\beta}{4}(t-T)} .
\end{aligned}
$$

De (4-59), temos que para todo $t \geq T$

$$
\begin{gathered}
\left\|\varphi(t)^{+}\right\|-\left\|\varphi^{-}(t)\right\| \geq\left\|\varphi^{-}(T)\right\| e^{\frac{\beta}{4}(t-T)} \\
\left\|\varphi(t)^{+}\right\|^{2}+\left\|\varphi^{-}(t)\right\|^{2} \geq\left\|\varphi^{-}(T)\right\|^{2} e^{\frac{\beta}{2}(t-T)} .
\end{gathered}
$$

Absurdo, pois de (4-58) temos que

$$
\left\|\varphi(t)^{+}\right\|^{2}+\left\|\varphi^{-}(t)\right\|^{2}<\delta_{1} \in \mathbb{R} \text { para todo } t \geq T .
$$

Mas

$$
\lim _{t \rightarrow \infty}\left(\left\|\varphi(t)^{+}\right\|^{2}+\left\|\varphi^{-}(t)\right\|^{2}\right)=\infty
$$

Desta forma, $\varphi$ não é estável e logo, pela Definição 4.1, $\varphi$ é instável.

Por fim, cabe destacar que, se o maior valor das partes reais dos autovalores de $A$ for igual a zero, nada podemos afirmar sobre a estabilidade do sistema, podendo este ser instável, estável ou até mesmo assintoticamente estável.

\section{3}

\section{Estabilidade em Sistemas Autônomos}

Nesta seção, analisaremos um caso particular do sistema (4-1) em que a função $F$ independe do instante $t$, ou seja, consideraremos o caso particular do seguinte sistema autônomo:

$$
x^{\prime}=F(x)
$$

em que $F: D \subseteq \mathbb{R}^{n} \rightarrow \mathbb{R}^{n}$ é uma função diferenciável na origem. 
Suporemos também que $F(0)=0 \in \mathbb{R}^{n}$ e logo a função identicamente nula é uma solução estacionária deste sistema.

Mostraremos como podemos escolher uma matriz real $A(n \times n)$ e uma função $f: D \rightarrow \mathbb{R}^{n}$ tal que

$$
F(x)=A x+f(x) \quad \forall x \in D
$$

e que

$$
\lim _{x \rightarrow 0} \frac{\|f(x)\|}{\|x\|}=0
$$

Como $F$ é diferenciável na origem, pela Definição B.34, seja $\varepsilon>0$ existe $\delta>0$ tal que se $\|x\| \leq \delta$ então

$$
\|F(0+x)-F(0)-D F(0) . x\| \leq \varepsilon\|x\|
$$

em que $D F(0)$ é a derivada de $F$ no ponto 0 .

Pelo Teorema B.35, $D F(0)$ é uma aplicação linear de $\mathbb{R}^{n}$ em $\mathbb{R}^{n}$ que está associada a uma matriz real $(n \times n)$ que denominaremos de $A$.

Definindo $f(x):=F(x)-A x$ para todo $x \in D$ e considerando que $F(0)=0$ temos que se $\|x\| \leq \delta$

$$
\|f(x)\|=\|F(x)-A x\| \leq \varepsilon\|x\| .
$$

Logo

$$
\lim _{x \rightarrow 0} \frac{\|f(x)\|}{\|x\|}=0
$$

Assim, o sistema

$$
x^{\prime}=A x+f(x)
$$

é equivalente ao sistema (4-79) e podemos utilizar os teoremas mostrados neste capítulo para analisar a estabilidade da solução identicamente nula deste sistema. 


\section{5 \\ Considerações Finais}

Iniciamos considerando algumas condições gerais para a existência e unicidade de soluções de quaisquer sistemas parametrizados $x^{\prime}=f(t, x, u)$, sejam eles lineares ou não.

Em seguida, estudamos os sistemas lineares $x^{\prime}=A(t) x+b(t)$ e suas propriedades e usamos os resultados num estudo sobre a estabilidade de soluções de sistemas não lineares, onde vimos o caso $x^{\prime}=A x+f(t, x)$ em que fixamos condições específicas para o comportamento de $f$ próximo a origem. Com isto, qualquer $\varphi$ que seja solução do sistema não-linear nos permite definir $b(t)=f(t, \varphi(t))$ e, como $\varphi^{\prime}(t)=A \varphi(t)+b(t)$, obtemos a equação de um sistema linear sendo possível utilizar o conhecimento que se tem sobre os sistemas lineares na análise dos sistemas não lineares.

Por fim, com a elaboração desta dissertação, foi possível aprofundar o conhecimento da Análise Real e aplicar os principais conceitos e resultados da Álgebra Linear. 


\section{Referências bibliográficas}

[1] ABBOTT, STEPHEN. Understanding Analysis. Springer, New York, 2nd edition, 2016.

[2] BARTLE, ROBERT. The Elements of Real Analysis. John Wiley and Sons, USA, 2nd edition, 1976.

[3] LIMA, ELON LAGES. Análise Real - Vol.1. IMPA, Rio de Janeiro, 12th edition, 2017.

[4] LIMA, ELON LAGES. Análise Real - Vol.2. IMPA, Rio de Janeiro, 6th edition, 2016.

[5] LIMA, ELON LAGES. Curso de Análise - Vol.2. IMPA, Rio de Janeiro, 11th edition, 2015.

[6] LANG, SERGE. Linear Algebra. Springer, New York, 3rd edition, 1987.

[7] QUARTERONI, ALFIO; SACCO, RICCARDO; SALERI, FAUSTO. Numerical Mathematics. Springer, New York, 2nd edition, 2007.

[8] DOERING, CLAUS I.; LOPES, ARTUR O.. Equações Diferenciais Ordinárias. IMPA, Rio de Janeiro, 6th edition, 2016.

[9] CODDINGTON, EARL A.; LEVISON, NORMAN. Theory of Ordinary Differential Equations. Tata McGraw-Hill Publishing Company Limited, New Delhi, tmh edition, 1972. 
A

\section{Apêndice - Demonstração de Resultados Utilizados}

Neste apêndice são demonstrados alguns resultados provenientes da Análise Real e Álgebra Linear utilizados neste estudo.

Proposição A.1 Sejam $\Pi \subset G \subseteq \mathbb{R}^{n}$ e $f: G \rightarrow \mathbb{R}^{m}$. Se $\Pi$ for compacto e $f$ for localmente Lipschitz em $G$ então $f$ é Lipschitz em $\Pi$.

Demonstração.

Pela Definição A.2, $f$ é Lipschitz em $\Pi$ se

$$
\exists L>0 \quad \forall x, y \in \Pi ;\|f(x)-f(y)\| \leq L\|x-y\| .
$$

Suponha por absurdo que $f$ não seja Lipschitz em П. Então, negando (A-1)

$$
\forall L>0 \quad \exists x, y \in \Pi ;\|f(x)-f(y)\|>L\|x-y\| .
$$

Note que, $x \neq y$ (caso contrário $\|f(x)-f(y)\|=0<L\|x-y\|=0$ ).

Assim, podemos construir as seguintes sequências $\left(x_{k}\right)_{k \in \mathbb{N}}$ e $\left(y_{k}\right)_{k \in \mathbb{N}}$

$$
\frac{\left\|f\left(x_{k}\right)-f\left(y_{k}\right)\right\|}{\left\|x_{k}-y_{k}\right\|}>k \text { em que } x_{k}, y_{k} \in \Pi \quad \forall k \in \mathbb{N} .
$$

Como $x_{k}$ e $y_{k} \in \Pi$ e $\Pi$ é compacto, pelo Teorema B.22, existe uma subsequência $\left(x_{k_{i}}\right)_{i \in \mathbb{N}}$ tal que $x_{k_{i}} \rightarrow x \in \Pi$ quando $i \rightarrow \infty$. Mas, pelo Teorema B.28, $f$ é limitada em $\Pi$ e logo, quando $i \rightarrow \infty$

$$
\frac{\left\|f\left(x_{k_{i}}\right)-f\left(y_{k_{i}}\right)\right\|}{\left\|x_{k_{i}}-y_{k_{i}}\right\|} \rightarrow \infty \Rightarrow\left\|x_{k_{i}}-y_{k_{i}}\right\| \rightarrow 0 \Rightarrow y_{k_{i}} \rightarrow x .
$$

Mas $f$ é localmente Lipschitz em $G$, logo existe $L_{x}>0$ e $\varepsilon>0$ tal que

$$
\text { se } z, w \in B_{\varepsilon}(x) \text { então }\|f(z)-f(w)\| \leq L_{x}\|z-w\| .
$$

Como $x_{k_{i}} \rightarrow x$ existe $N_{1} \in \mathbb{N}$ tal que $x_{k_{i}} \in B_{\varepsilon}(x)$ para todo $i>N_{1}$.

Como $y_{k_{i}} \rightarrow x$ existe $N_{2} \in \mathbb{N}$ tal que $y_{k_{i}} \in B_{\varepsilon}(x)$ para todo $i>N_{2}$.

Absurdo, pois seja $N=\max \left\{N_{1}, N_{2}, L_{x}\right\}$, de (A-3) e (A-5), para todo $i>N$

$$
L_{x} \leq N<i \leq k_{i}<\frac{\left\|f\left(x_{k_{i}}\right)-f\left(y_{k_{i}}\right)\right\|}{\left\|x_{k_{i}}-y_{k_{i}}\right\|}<L_{x} \Rightarrow L_{x}<L_{x} .
$$

\footnotetext{
${ }^{1}$ Também podemos demonstrar esta proposição utilizando as definições de compacto e coberturas [2] e concluir que existe um número finito de vizinhanças que cobrem $\Pi$ e, assim, definimos $L$ como o máximo entre as constantes de Lipschitz de cada uma destas vizinhanças.
} 
Proposição A.2 Seja $G \subseteq \mathbb{R}^{1+n}$ aberto e $f: G \rightarrow \mathbb{R}^{m}$ diferenciável em $G$ tal que $\left|\frac{\partial f_{i}}{\partial x_{j}}(t, x)\right| \leq M$ para todo $(t, x) \in G, i \leq m$ e $j \leq n$ então $f$ é Lipschitz com respeito a $x$ em $G$.

Demonstração.

Sejam $G_{1} \times G_{2}:=G$ e $t \in G_{1}$ (fixo), $x$ e $y \in G_{2}$ (arbitrários), definimos $g_{i}: G_{2} \rightarrow \mathbb{R}$ em que $g_{i}(x):=f_{i}(t, x) \Rightarrow\left|\frac{\partial g_{i}}{\partial x_{j}}(x)\right| \leq M$ para todo $i$ e $j$.

Pelo Teorema do Valor Médio B.37, existe um ponto $\alpha_{i} \in G_{2}$ localizado no segmento de reta que liga o ponto $x$ ao ponto $y$ tal que para todo $i \leq n$ temos:

$$
\begin{aligned}
g_{i}(x)-g_{i}(y) & =\nabla g_{i}\left(\alpha_{i}\right) \cdot(x-y) \\
\left|g_{i}(x)-g_{i}(y)\right| & \leq\left|\sum_{j=1}^{n}\left(x_{j}-y_{j}\right) \frac{\partial g_{i}}{\partial x_{j}}\left(\alpha_{i}\right)\right| \leq \sum_{j=1}^{n}\left|x_{j}-y_{j}\right|\left|\frac{\partial g_{i}}{\partial x_{j}}\left(\alpha_{i}\right)\right| .
\end{aligned}
$$

Logo

$$
\begin{gathered}
\left|f_{i}(t, x)-f_{i}(t, y)\right| \leq M \sum_{j=1}^{n}\left|x_{j}-y_{j}\right|=M\|x-y\|_{1} \\
\|f(t, x)-f(t, y)\|_{1} \leq M \sum_{i=1}^{m}\|x-y\|_{1}=M m\|x-y\|_{1} .
\end{gathered}
$$

Como $x$ e $y$ são arbitrários, existe $L_{1}:=M m>0$ para todo $x, y \in G_{2}$ tal que

$$
\|f(t, x)-f(t, y)\|_{1} \leq L_{1}\|x-y\|_{1}
$$

Pela equivalência das normas em $\mathbb{R}^{n}$ (Teorema B.9) e pela Definição B.29 temos que $f$ é Lipschitz com respeito a $x$ em $G$.

Corolário A.3 Sejam $\frac{\partial f_{i}}{\partial x_{j}}(t, x)$ contínuas em $G$ para todo $i \leq m$ e $j \leq n$ então $f$ é localmente Lipschitz com respeito a $x$ em $G$.

Demonstração.

Seja $(t, x) \in G$ arbitrário então, como $G$ é aberto, existe $\varepsilon>0$ tal $K:=\bar{B}_{\varepsilon}(t, x) \subset G$, ou seja, existe uma bola fechada de raio $\varepsilon$ centrada em $(t, x)$ que está contida em $G$. Pelo Teorema B.14, $K$ é compacto.

Como as derivadas parciais $\frac{\partial f_{i}}{\partial x_{j}}$ são contínuas em $K$ compacto para todo $i$ e $j$ então, por B.28, $\frac{\partial f_{i}}{\partial x_{j}}$ são limitadas e podemos aplicar o Teorema A.2 em $K$.

Mas $(t, x)$ é arbitrário então para todo $(t, x) \in G$ existe uma vizinhança em $K$ na qual $f$ é Lipschitz com respeito a $x$ nesta vizinhança. Pela Definição B.29, temos que $f$ é localmente Lipschitz com respeito a $x$ em $G$. 


\section{Teorema A.4 (Teorema de Weierstrass para Convergência Uniforme)}

Seja $\left\{f_{k}\right\}_{k \in \mathbb{N}}$ uma sequência de funções $f_{k}: G \subseteq \mathbb{R}^{n} \rightarrow \mathbb{R}^{m}$ tal que $\left\|f_{k}(x)\right\| \leq M_{k} \in \mathbb{R}$ para todo $k \in \mathbb{N}$ e $x \in G$.

Se $\sum_{k=1}^{\infty} M_{k}$ converge então $\sum f_{k}$ converge uniformemente em $G$.

Demonstração.

Como $\sum M_{k}$ converge, pelo critério de Cauchy (Teorema B.18), seja $\varepsilon>0$ existe $N \in \mathbb{N}$ tal que para todo $i \geq j \geq N$ temos $\sum_{k=j}^{i} M_{k}<\frac{\varepsilon}{2}$.

Assim, seja $x \in G$ temos

$$
\frac{\varepsilon}{2}>\sum_{k=j}^{i} M_{k} \geq \sum_{k=j}^{i}\left\|f_{k}(x)\right\| \geq\left\|\sum_{k=j}^{i} f_{k}(x)\right\| .
$$

Como $x$ é arbitrário temos que $\sup _{x \in G}\left\|\sum_{k=j}^{i} f_{k}(x)\right\| \leq \frac{\varepsilon}{2}<\varepsilon$.

Pelo critério de convergência uniforme de Cauchy (Teorema B.44) temos que a série $\sum f_{k}$ converge uniformemente em $G$.

Corolário A.5 Este teorema também é aplicável a funções matriciais, utilizando normas matriciais.

\section{Proposição A.6 (Convergência Uniforme de Funções Compostas)}

Sejam $G \subseteq \mathbb{R}^{n}$ e $D \subseteq \mathbb{R}^{p}$ compactos, $\left\{f_{k}\right\}_{k \in \mathbb{N}}$ e $\left\{x_{k}\right\}_{k \in \mathbb{N}}$ sequências de funções contínuas $f_{k}: G \rightarrow \mathbb{R}^{m}$ e $x_{k}: D \rightarrow G$ tal que $f_{k} \rightarrow f$ uniformemente em $G$ e $x_{k} \rightarrow x$ uniformemente em $D$ então a sequência composta $\left\{g_{k}\right\}_{k \in \mathbb{N}}$, dada por $g_{k}: D \rightarrow \mathbb{R}^{m}$ com $g_{k}=f_{k}\left(x_{k}\right)$, converge uniformemente em $D$ para $g=f(x)$.

Demonstração.

Pelas definições de convergência uniforme (Definição B.41) e continuidade uniforme (Definição B.27) temos

1. $x_{k} \rightarrow x$ uniformemente em $D$ então

$$
\forall \delta>0 \exists N_{1} \forall k>N_{1} \forall t \in D \quad\left\|x_{k}(t)-x(t)\right\|<\delta .
$$

2. $f_{k} \rightarrow f$ uniformemente em $G$ então

$$
\forall \varepsilon>0 \exists N_{2} \forall k>N_{2} \forall y \in G\left\|f_{k}(y)-f(y)\right\|<\varepsilon .
$$

3. $f_{k}$ contínua em $G$ compacto então, pelo Teorema B.30, $f_{k}$ é uniformemente contínua em $G$

$$
\forall \varepsilon>0 \exists \delta>0 \forall x, y \in G\|y-x\|<\delta \Rightarrow\left\|f_{k}(y)-f_{k}(x)\right\|<\frac{\varepsilon}{2} .
$$


Assim, seja $\varepsilon>0$ de (A-13) temos que existe $\delta>0$ e por (A-11) existe $N_{1}$ tal que para todo $k>N_{1}$ se $\left\|x_{k}(t)-x(t)\right\|<\delta$ então

$$
\left\|f_{k}\left(x_{k}(t)\right)-f_{k}(x(t))\right\|<\frac{\varepsilon}{2} .
$$

De (A-12), existe $N_{2}$ tal que para todo $k>N_{2}$ e $t \in D$

$$
\left\|f_{k}\left(x_{k}(t)\right)-f\left(x_{k}(t)\right)\right\|<\frac{\varepsilon}{2} .
$$

Seja $N=\max \left\{N_{1}, N_{2}\right\}$ então para todo $k>N$ e $t \in D$

$$
\begin{aligned}
\left\|g_{k}(t)-g(t)\right\| & =\left\|f_{k}\left(x_{k}(t)\right)-f(x(t))\right\| \\
& =\left\|f_{k}\left(x_{k}(t)\right)-f_{k}(x(t))+f_{k}(x(t))-f(x(t))\right\| \\
& \leq\left\|f_{k}\left(x_{k}(t)\right)-f_{k}(x(t))\right\|+\left\|f_{k}(x(t))-f(x(t))\right\| \\
& <\frac{\varepsilon}{2}+\frac{\varepsilon}{2}=\varepsilon \quad(\text { por }(\mathrm{A}-14) \text { e }(\mathrm{A}-15)) .
\end{aligned}
$$

Assim, $g_{k}$ converge para $g$ uniformemente em $D$.

Proposição A.7 (Lema de Hadamard) Seja $g: D \subseteq \mathbb{R}^{n+m} \rightarrow \mathbb{R}$ em que $g \in C^{1}(D)$ então existem funções contínuas $\psi_{i}(x, y, z)$ tal que para todo $(x, y)$ $e(x, z) \in D$ temos:

$$
g(x, y)-g(x, z)=\sum_{i=1}^{n}\left(y_{i}-z_{i}\right) \psi_{i}(x, y, z) .
$$

Demonstração.

Sejam $w(t):=z+t(y-z)$ e $\alpha:=g(x, w(t))$ então pelo Teorema Fundamental do Cálculo B.40

$$
\begin{aligned}
g(x, y)-g(x, z) & =\alpha(1)-\alpha(0)=\int_{0}^{1} \alpha^{\prime}(t) d t \\
& =\sum_{i=1}^{m}\left[\int_{0}^{1} \frac{\partial g}{\partial w_{i}}(x, w(t)) d t\right]\left(z_{i}-y_{i}\right) \\
& =: \sum_{i=1}^{m}\left(z_{i}-y_{i}\right) \psi_{i}(x, y, z) \\
\text { em que } \psi_{i}(x, y, z) & :=\int_{0}^{1} \frac{\partial g}{\partial w_{i}}(x, w(t)) d t \quad(i=1 \ldots m) .
\end{aligned}
$$

Como $g \in C^{1}(D)$ então $\frac{\partial g}{\partial w_{i}}$ é contínua para todo $i=1 \ldots m$ e, pelo Teorema Fundamental do Cálculo B.40, $\psi_{i}$ também são contínuas. 
Proposição A.8 Seja $K \subset \mathbb{R}^{n}$ compacto e $F \subset \mathbb{R}^{n}$ fechado tal que $K \cap F=\emptyset$ então existem $x_{0} \in K$ e $y_{0} \in F$ tal que

$$
\left\|x_{0}-y_{0}\right\|=\inf _{\substack{x \in K \\ y \in F}}\|x-y\|=: \operatorname{dist}(K, F)>0 .
$$

Demonstração.

Seja $d:=\inf _{\substack{x \in K \\ y \in F}}\|x-y\|$ então pela definição de ínfimo

$$
\forall \varepsilon>0 \exists x \in K \exists y \in F \quad d \leq\|x-y\|<d+\varepsilon .
$$

Assim, seja $\left(\varepsilon_{k}\right)_{k \in \mathbb{N}}$ a sequência em que $\varepsilon_{k}=\frac{1}{k}$ então existem $x_{k} \in K$ e $y_{k} \in F$ para todo $k \in \mathbb{N}$ tais que

$$
d \leq\left\|x_{k}-y_{k}\right\|<d+\varepsilon_{k}=d+\frac{1}{k} .
$$

Como $x_{k} \in K$ e $K$ é compacto então, pelo Teorema B.22, existe uma subsequência $\left(x_{k_{i}}\right)_{i \in \mathbb{N}}$ tal que $x_{k_{i}} \rightarrow x_{0}$ para algum $x_{0} \in K$. Pela definição de limite, existe $i_{0} \in \mathbb{N}$ tal que para todo $k_{i}>k_{i_{0}}$ temos $\left\|x_{k_{i}}\right\|<\left\|x_{0}\right\|+1$.

De $(\mathrm{A}-18)$ temos para todo $k_{i}>k_{i_{0}}$

$$
\left\|y_{k_{i}}\right\|<d+\frac{1}{k_{i}}+\left\|x_{k_{i}}\right\|<d+\frac{1}{k_{i_{0}}}+\left\|x_{k_{i}}\right\|<d+\frac{1}{k_{i_{0}}}+\left\|x_{0}\right\|+1=: M \in \mathbb{R} .
$$

Assim $\left(y_{k_{i}}\right)_{i \in \mathbb{N}}$ é uma sequência limitada e, pelo Teorema B.22, existe uma subsequência convergente $\left(y_{k_{i j}}\right)_{j \in \mathbb{N}}$ tal que $y_{k_{i_{j}}} \rightarrow y_{0}$ quando $j \rightarrow \infty$. Como $F$ é fechado temos que $y_{0} \in F$.

Mas $K \cap F=\emptyset$ então $x_{0} \neq y_{0}$ e, por (A-18) e pelo Teorema B.23

$$
d=\lim _{k \rightarrow \infty}\left\|x_{k}-y_{k}\right\|=\lim _{j \rightarrow \infty}\left\|x_{k_{i_{j}}}-y_{k_{i_{j}}}\right\|=\left\|x_{0}-y_{0}\right\|>0 .
$$


Proposição A.9 Sejam $K \subset G \subseteq \mathbb{R}^{n} ; G$ aberto; $K$ compacto; $d_{0}:=\operatorname{dist}(K, \partial G)$ e $K^{\prime}:=\left\{y \in \mathbb{R}^{n} ; \operatorname{dist}(y, K):=\inf _{x \in K}\|y-x\| \leq \frac{d_{0}}{2}\right\}$ então $K^{\prime}$ é compacto e $K \subset K^{\prime} \subset G$.

Demonstração.

Inicialmente, note que, pela Proposição A.8 temos $d_{0}>0$ e logo $K^{\prime} \neq \emptyset$.

Afirmação (I): $K^{\prime}$ é limitado.

Por absurdo, suponha que não. Como $K$ é compacto então, pelo Teorema de Heine-Borel B.14, $K$ é limitado e logo existe $M>0$ tal que $\|y\| \leq M$ para todo $y \in K$. Já que $K^{\prime}$ é ilimitado, existe $x \in K^{\prime}$ tal que $\|x\|>M+d_{0}$.

Seja $y \in K \Rightarrow\|x-y\| \geq\|x\|-\|y\|>M+d_{0}-M=d_{0}$.

Como $y$ é arbitrário: $\inf _{y \in K}\|x-y\| \geq d_{0}>\frac{d_{0}}{2} \Rightarrow x \notin K^{\prime}$. Absurdo.

Afirmação (II): $K^{\prime}$ é fechado.

Seja $x_{0} \in \partial K^{\prime}$ então pela Definição de Fronteira B.12:

$$
\begin{gathered}
\forall \varepsilon>0 \exists x \in K^{\prime}\left\|x-x_{0}\right\|<\varepsilon \Rightarrow \forall k \in \mathbb{N} \exists x_{k} \in K^{\prime}\left\|x_{k}-x_{0}\right\|<\frac{1}{k} \\
x_{k} \in K^{\prime} \Rightarrow \operatorname{dist}\left(x_{k}, K\right) \leq \frac{d_{0}}{2} .
\end{gathered}
$$

Quando $k \rightarrow \infty$ temos $x_{k} \rightarrow x_{0}$ então pela continuidade da função dist (Proposição B.15) e pelo Teorema B.23

$$
\operatorname{dist}\left(x_{0}, K\right)=\lim _{k \rightarrow \infty} \operatorname{dist}\left(x_{k}, K\right) \leq \frac{d_{0}}{2}
$$

Logo $x_{0} \in K^{\prime}$ e como $x_{0}$ é arbitrário então $\partial K^{\prime} \subset K^{\prime}$ e portanto $K^{\prime}$ é fechado e como também é limitado então, pelo Teorema B.14, $K^{\prime}$ é compacto.

Afirmação (III): $K \subset K^{\prime}$.

Seja $x \in K$ (arbitrário) então

$$
\operatorname{dist}(x, K)=0 \leq \frac{d_{0}}{2} .
$$

Logo $x \in K^{\prime}$ e portanto $K \subset K^{\prime}$.

Afirmação (IV): $K^{\prime} \subset G$.

Por absurdo, suponha que não. Assim, existe $x \in K^{\prime}$ tal que $x \notin G$ e logo $\operatorname{dist}(x, K) \geq d_{0}$. Absurdo, pois $x \in K^{\prime} \operatorname{logo} \operatorname{dist}(x, K) \leq \frac{d_{0}}{2}$. Desta forma, $x \in G$ e com isto $K^{\prime} \subset G$. 


\section{Teorema A.10 (Derivada de Determinantes)}

Seja $A(t):=\left[\begin{array}{ccc}a_{11}(t) & \ldots & a_{1 n}(t) \\ a_{21}(t) & \ldots & a_{2 n}(t) \\ \ldots & \ldots & \ldots \\ a_{n 1}(t) & \ldots & a_{n n}(t)\end{array}\right]$ em que para todo $i, j=1 \ldots n$

$a_{i j}(t)$ são funções diferenciáveis de $I \subseteq \mathbb{R} \rightarrow \mathbb{R}$ então:

$$
|A(t)|^{\prime}=\left|\begin{array}{ccc}
a_{11}^{\prime}(t) & \ldots & a_{1 n}^{\prime}(t) \\
a_{21}(t) & \ldots & a_{2 n}(t) \\
\ldots & \ldots & \ldots \\
a_{n 1}(t) & \ldots & a_{n n}(t)
\end{array}\right|+\left|\begin{array}{ccc}
a_{11}(t) & \ldots & a_{1 n}(t) \\
a_{21}^{\prime}(t) & \ldots & a_{2 n}^{\prime}(t) \\
\ldots & \ldots & \ldots \\
a_{n 1}(t) & \ldots & a_{n n}(t)
\end{array}\right|+\cdots+\left|\begin{array}{ccc}
a_{11}(t) & \ldots & a_{1 n}(t) \\
a_{21}(t) & \ldots & a_{2 n}(t) \\
\ldots & \ldots & \ldots \\
a_{n 1}^{\prime}(t) & \ldots & a_{n n}^{\prime}(t)
\end{array}\right| .
$$

Demonstração.

Sejam $h \neq 0, A_{i}:=A_{i}(t)$ a i-ésima linha da matriz $A(t)$ para todo $i \leq n$ e a matriz $A^{h}:=A(t+h)$ em que $A_{i}^{h}:=A_{i}(t+h)$ para todo $i \leq n$.

Como a função determinante $D$ é multilinear (pelo Teorema B.51) então

$$
\begin{aligned}
\operatorname{det}\left(A^{h}\right)=\left|A^{h}\right| & =D\left(A_{1}^{h}, A_{2}^{h}, \ldots, A_{n}^{h}\right) \\
& =D\left(A_{1}^{h}-A_{1}+A_{1}, A_{2}^{h}, \ldots, A_{n}^{h}\right) \\
& =D\left(A_{1}^{h}-A_{1}, A_{2}^{h}, \ldots, A_{n}^{h}\right)+D\left(A_{1}, A_{2}^{h}, \ldots, A_{n}^{h}\right)\left(^{*}\right) \\
& =D_{1}+D\left(A_{1}, A_{2}^{h}, \ldots, A_{n}^{h}\right)
\end{aligned}
$$

em que $D_{1}:=D\left(A_{1}^{h}-A_{1}, A_{2}^{h}, \ldots, A_{n}^{h}\right)$ e a igualdade $\left(^{*}\right)$ é dada pela multilinearidade de $D$. Analogamente, definimos $D_{2}$ a seguir:

$$
\begin{aligned}
D\left(A_{1}, A_{2}^{h}, \ldots, A_{n}^{h}\right) & =D\left(A_{1}, A_{2}^{h}-A_{2}+A_{2}, \ldots, A_{n}^{h}\right) \\
& =D\left(A_{1}, A_{2}^{h}-A_{2}, \ldots, A_{n}^{h}\right)+D\left(A_{1}, A_{2}, A_{3}^{h} \ldots, A_{n}^{h}\right) \\
& =: D_{2}+D\left(A_{1}, A_{2}, A_{3}^{h} \ldots, A_{n}^{h}\right) .
\end{aligned}
$$

Procedemos desta forma até definir $D_{n}$

$$
\begin{aligned}
D\left(A_{1}, \ldots, A_{n-1}, A_{n}^{h}\right) & =D\left(A_{1}, \ldots, A_{n-1}, A_{n}^{h}-A_{n}\right)+D\left(A_{1}, \ldots, A_{n}\right) \\
& =: D_{n}+D\left(A_{1}, \ldots, A_{n}\right) .
\end{aligned}
$$

Assim temos

$$
\begin{aligned}
\left|A^{h}\right|=|A(t+h)| & =D_{1}+\cdots+D_{n}+D\left(A_{1}, \ldots, A_{n}\right) \\
& =D_{1}+\cdots+D_{n}+|A(t)| \\
\frac{|A(t+h)|-|A(t)|}{h} & =\frac{1}{h} \sum_{i=1}^{n} D_{i} .
\end{aligned}
$$


Pela multilinearidade de $D$, sejam $B$ matriz $(n \times n)$ qualquer e $\alpha \in \mathbb{R}$ então para todo $i \leq n$ temos

$$
\alpha D\left(B_{1}, \ldots, B-n\right)=D\left(B_{1}, \ldots, B_{i-1}, \alpha B_{i}, B_{i+1}, \ldots, B_{n}\right) .
$$

Assim, tomando $\alpha=\frac{1}{h}$ em (A-29) então por (A-29) temos

$$
\frac{|A(t+h)|-|A(t)|}{h}=\sum_{i=1}^{n} D\left(A_{1}, \ldots, A_{i-1}, \frac{A_{i}^{h}-A_{i}}{h}, A_{i+1}^{h}, \ldots, A_{n}^{h}\right)
$$

Quando $h \rightarrow 0$ como $A_{j}$ é contínua então $A_{j}^{h}=A_{j}(t+h) \rightarrow A_{j}(t)=A_{j}$ para todo $i<j \leq n$ e como $D$ também é contínua temos

$$
\begin{aligned}
|A(t)|^{\prime} & =\lim _{h \rightarrow 0} \frac{|A(t+h)|-|A(t)|}{h} \\
& =\sum_{i=1}^{n} D\left(A_{1}, \ldots, A_{i-1}, \lim _{h \rightarrow 0} \frac{A_{i}^{h}-A_{i}}{h}, \lim _{h \rightarrow 0} A_{i+1}^{h}, \ldots, \lim _{h \rightarrow 0} A_{n}^{h}\right) \\
& =\sum_{i=1}^{n} D\left(A_{1}(t), \ldots, A_{i-1}(t), \lim _{h \rightarrow 0} \frac{A_{i}(t+h)-A_{i}(t)}{h}, A_{i+1}(t), \ldots, A_{n}(t)\right) \\
& =\sum_{i=1}^{n} D\left(A_{1}(t), \ldots, A_{i-1}(t), A_{i}^{\prime}(t), A_{i+1}(t), \ldots, A_{n}(t)\right) .
\end{aligned}
$$

\section{Proposição A.11 (Continuidade da Matriz Inversa)}

Seja $A(x):=\left(a_{i j}(x)\right)_{i, j}^{n}$ em que $a_{i j}: X \subseteq \mathbb{R}^{n} \rightarrow \mathbb{R}$ são funções contínuas $e|A(x)| \neq 0$ para todo $x \in X$ então existe função $A^{-1}$ contínua tal que $A^{-1}(x) A(x)=I$ para todo $x \in X$.

\section{Demonstração.}

Como $\operatorname{det}(A(x)) \neq 0$ para todo $x \in X$ então, pelo Teorema B.53, para todo $x \in X$ existe uma matriz inversa $A^{-1}(x)=\left(b_{i j}(x)\right)_{i, j}^{n}$ tal que $A^{-1}(x) A(x)=I$.

Do Teorema B.52, temos para todo $x \in X$

$$
b_{i j}(x)=\frac{D\left(A_{1}(x), \ldots, e_{j}, \ldots, A_{n}(x)\right)}{D(A(x))}
$$

em que $A_{i}(x)=\left(a_{i 1}(x), \ldots, a_{i n}(x)\right)$ para todo $i \leq n$.

Como a função determinante $D$ é contínua (pelo Teorema B.51) e $A_{i}$ são contínuas para todo $i \leq n$ então $b_{i j}$ são composições de funções contínuas para todo $i, j \leq n$ e logo, pelo Teorema B.30, $b_{i j}$ são contínuas e, portanto, $A^{-1}=\left(b_{i j}\right)_{i, j=1}^{n}$ também é contínua. 
Proposição A.12 Sejam $w$, y e z funções reais contínuas em $I=[a, b] \subset \mathbb{R}$ tais que para todo $t \in I$ tivermos $z(t) \geq 0$ e

$$
w(t) \leq y(t)+\int_{a}^{t} z(s) w(s) d s
$$

então para todo $t \in I$ temos

$$
w(t) \leq y(t)+\int_{a}^{t} z(s) y(s) e^{\int_{s}^{t} z(p) d p} d s
$$

Demonstração.

Seja a função real $R$ contínua em $I$ definida a seguir para todo $t \in I$

$$
R(t):=\int_{a}^{t} z(s) w(s) d s \Rightarrow w(t) \leq y(t)+R(t) .
$$

Pelo Teorema Fundamental do Cálculo B.40 temos que para todo $s \in I$

$$
R^{\prime}(s):=z(s) w(s)
$$

De $(\mathrm{A}-32)$ e (A-33)

$$
\begin{aligned}
R^{\prime}(s)-z(s) R(s) & =z(s) w(s)-z(s) R(s) \\
& \leq z(s)(y(s)+R(s))-z(s) R(s)=z(s) y(s) .
\end{aligned}
$$

Seja $t \in I$ (fixo), multiplicando (A-34) por $e^{\int_{s}^{t} z(p) d p}>0$ temos para todo $s \in I$

$$
\left(R^{\prime}(s)-z(s) R(s)\right) e^{\int_{s}^{t} z(p) d p} \leq z(s) y(s)^{\int_{s}^{t} z(p) d p} .
$$

Integrando de $a$ a $t$ temos

$$
\int_{a}^{t} R^{\prime}(s) e^{\int_{s}^{t} z(p) d p} d s-\int_{a}^{t} z(s) R(s) e^{\int_{s}^{t} z(p) d p} d s \leq \int_{a}^{t} z(s) y(s)^{\int_{s}^{t} z(p) d p} d s .
$$

Integrando por partes $\int_{a}^{t} R^{\prime}(s) e^{\int_{s}^{t} z(p) d p}$ temos

$$
\begin{aligned}
u^{\prime} & =R^{\prime} \Rightarrow u=R \quad v=e^{-\int_{t}^{s} z(p) d p} \Rightarrow v^{\prime}=z e^{-\int_{t}^{s} z(p) d p} \\
\int_{a}^{t} R^{\prime}(s) e^{\int_{s}^{t} z(p) d p} d s & =R(t) e^{0}-R(a) e^{-\int_{t}^{a} z(p) d p}+\int_{a}^{t} z(s) R(s) e^{-\int_{t}^{s} z(p) d p} d s .
\end{aligned}
$$

Mas $R(a)=0$ e $\log \mathrm{o}$

$$
\int_{a}^{t} R^{\prime}(s) e^{\int_{s}^{t} z(p) d p} d s=R(t)+\int_{a}^{t} z(s) R(s) e^{\int_{s}^{t} z(p) d p} d s .
$$

De $(\mathrm{A}-36)$

$$
R(t) \leq \int_{a}^{t} z(s) y(s) e^{\int_{s}^{t} z(p) d p} d s
$$

Por fim, de (A-32) temos

$$
w(t) \leq y(t)+R(t) \leq y(t)+\int_{a}^{t} z(s) y(s) e^{\int_{s}^{t} z(p) d p} d s .
$$


B

\section{Apêndice - Análise Real e Álgebra Linear}

A seguir são enunciadas as principais definições, proposições e teoremas de Análise Real e Álgebra Linear relacionados com assuntos utilizados neste estudo, cujas demonstrações podem ser obtidas nas respectivas citações bibliográficas.

\section{B.1}

\section{Espaço Vetorial}

Definição B.1 (Campo) [6]

Dizemos que $K \subseteq \mathbb{C}$ é um campo se satisfaz as seguintes condições:

1. se $x, y \in K$ então $(x+y) \in K$ e $x y \in K$;

2. se $x \in K$ então $(-x) \in K$ e se $x \neq 0$ então $\frac{1}{x} \in K$ e;

3. os elementos 0 e 1 são elementos de $K$.

Naturalmente, $\mathbb{R}$ e $\mathbb{C}$ são exemplos de campos.

\section{Definição B.2 (Espaço Vetorial) [6]}

Dizemos que $V$ é um espaço vetorial sobre o campo $K$ se satisfaz as seguintes propriedades:

1. dados $u, v, w \in V$ então $(u+v)+w=u+(v+w)$;

2. existe um elemento $0 \in V$ tal que $0+u=u+0=u$;

3. dado $u \in V$, existe um elemento $-u \in V$ tal que $u+(-u)=0$;

4. para todo $u, v \in V$ então $(u+v)=(v+u) \in V$;

5. se $c \in K$ e $u, v \in V$ então $c(u+v)=c u+c v \in V$;

6. se $a, b \in K$ e $v \in V$ então $(a b) v=a(b v) e$;

7. para todo elemento $u \in V$ e $1 \in K$ tem-se $1 . u=u$. 
São exemplos de espaços vetoriais:

- $K^{n}$ (onde $K$ é um campo qualquer como, por exemplo, $\mathbb{R}$ e $\mathbb{C}$ );

- o conjunto das matrizes $(m \times n)$ com elementos em $K$ e;

- o conjunto das funções contínuas de $G \subseteq \mathbb{R}^{n} \rightarrow \mathbb{R}^{m}$.

Neste estudo consideraremos $K=\mathbb{R}$ e diremos simplesmente que $V$ é um espaço vetorial.

\section{Definição B.3 (Subespaço Vetorial) [6]}

Seja $V$ um espaço vetorial sobre o campo $K$ e $W \subset V$, dizemos que $W$ é um subespaço de $V$ se satisfaz as seguintes propriedades:

1. Se $v, w \in W$ então $(u+v) \in W$ e;

2. Se $v \in W$ e c $\in K$ então $(c v) \in W$.

Assim, pela definição B.2, todo subespaço vetorial é um espaço vetorial.

\section{Definição B.4 (Vetores Linearmente Independentes) [6]}

Sejam $V$ um espaço vetorial sobre um campo $K$ e $v_{1}, \ldots, v_{n}$ elementos de $V$, dizemos que $v_{1}, \ldots, v_{n}$ são linearmente dependentes em $K$, se existirem $a_{1}, \ldots, a_{n}$ elementos de $K$, nem todos nulos tais que $a_{1} v_{1}+\ldots a_{n} v_{n}=0$.

Caso contrário, dizemos que $v_{1}, \ldots, v_{n}$ são linearmente independentes, ou seja:

$$
a_{1} v_{1}+\ldots a_{n} v_{n}=0 \Rightarrow a_{1}=\cdots=a_{n}=0 \text {. }
$$

\section{Teorema B.5 (Base de um Espaço Vetorial) [6]}

Sejam $v_{1}, \ldots, v_{n}$ elementos linearmente independentes de um espaço vetorial $V$ sobre um campo $K$, em que a dimensão de $V$ é igual a $n$, então $v_{1}, \ldots, v_{n}$ formam uma base geradora deste espaço tal que para todo $v \in V$ existe um único $c \in K^{n}$, denominado de vetor de coordenadas de $v$ nesta base, tal que:

$$
v=\sum_{i=1}^{n} c_{i} v_{i}
$$

Reciprocamente, para todo $c \in K^{n}$ existe um único $v \in V$ dado por (I). 


\section{B.2}

\section{Normas}

Definição B.6 (Norma) [2, 4, 7]

Seja $V$ um espaço vetorial em $K$, então uma norma em $V$ é uma função de $V$ em $\mathbb{R}$ denotada por $\|$.$\| que satisfaz:$

1. $\|x\| \geq 0$ para todo $x \in V$ e $\|x\|=0$ se e somente se $x=0$;

2. $\|a x\|=|a|\|x\|$ para todo $a \in K$ e $x \in V$ e;

3. $\|x+y\| \leq\|x\|+\|y\|$.

Note que $|x|$ é uma norma em $\mathbb{R}$ e $\mathbb{C}$. São exemplos de normas em $\mathbb{R}^{n}$ e $\mathbb{C}^{n}$ :

$$
\begin{aligned}
& -\|x\|_{2}=\sqrt{\left|x_{1}\right|^{2}+\left|x_{2}\right|^{2}+\cdots+\left|x_{n}\right|^{2}} \\
& -\|x\|_{1}=\left|x_{1}\right|+\left|x_{2}\right|+\cdots+\left|x_{n}\right| e ; \\
& -\|x\|_{\infty}=\max \left\{\left|x_{1}\right|,\left|x_{2}\right|, \ldots,\left|x_{n}\right|\right\} .
\end{aligned}
$$

No caso do espaço vetorial das matrizes $(n \times n)$ (reais ou complexas) podemos definir como norma de uma matriz $A=\left(a_{i j}\right)_{i, j=1}^{n}$ :

$$
\|A\|_{F}=\sqrt{\sum_{i, j}\left|a_{i j}\right|^{2}} \quad \text { (Norma Frobenius / Euclidiana em } \mathbb{C}^{n^{2}} \text {. }
$$

\section{Proposição B.7 (Desigualdades de Normas) [2, 4, 6]}

Sejam $V$ espaço vetorial e $x, y \in V$ então:

1. $|x . y| \leq\|x\|\|y\|$ [Desigualdade de Schwarz para produtos internos] e;

2. | $\|x\|-\|y\| \mid \leq\|x \pm y\| \leq\|x\|+\|y\|$ [Desigualdade Triangular].

\section{Teorema B.8 (Continuidade da Norma) [5, 7]}

Seja \|.\| uma norma qualquer em $\mathbb{R}^{n}$ então $\|$.$\| é uniformemente contínua.$

\section{Teorema B.9 (Equivalência de Normas em $\mathbb{R}^{n}$ ) [5, 7, 8]}

Todas as normas em $\mathbb{R}^{n}$ são equivalentes, ou seja, sejam $\|\cdot\|_{\alpha}$ e $\|\cdot\|_{\beta}$ normas quaisquer em $\mathbb{R}^{n}$ então existem $a, b>0$ tais que:

$$
a\|\cdot\|_{\alpha} \leq\|\cdot\|_{\beta} \leq b\|\cdot\|_{\alpha}
$$

e, como consequência, temos que todas as normas matriciais induzidas por uma norma vetorial qualquer em $\mathbb{R}^{n}$ são equivalentes. 
Definição B.10 (Norma Matricial Induzida) [5, 7, 8]

Seja A uma matriz $n \times m$ com elementos em $K=\mathbb{R}($ ou $\mathbb{C})$, definimos a seguinte norma matricial induzida por uma norma vetorial qualquer:

$$
\|A\|=\sup \left\{\|A x\|: x \in K^{m} ;\|x\|=1\right\}
$$

(note que deve-se utilizar a mesma norma vetorial escolhida em $\|x\| e\|A x\|$ ). Esta norma, possui as propriedades definidas em B.6 e B.7.2, bem como:

1. Compatibilidade com a norma vetorial $\|x\|$ :

$\|A x\| \leq\|A\|\|x\|$ para todo $x \in K^{m}$.

2. Propriedade Sub-Multiplicativa:

$\|A B\| \leq\|A\|\|B\|$ para $B$ matriz $(m \times k) \Rightarrow\left\|A^{k}\right\| \leq\|A\|^{k} \quad \forall k \in \mathbb{N}$.

3. $\|I\|=1$.

Por exemplo, adotando as normas vetoriais $\|\cdot\|_{\infty}$ ou $\|\cdot\|_{1}$, podemos calcular facilmente a respectiva norma induzida em função dos elementos $a_{i j}$ de $A$ :

$$
\|A\|_{\infty}=\max _{i} \sum_{j}\left|a_{i j}\right| \quad e \quad\|A\|_{1}=\max _{j} \sum_{i}\left|a_{i j}\right| .
$$

Note que a norma Frobenius definida na Definição B.6 também goza das propriedades 1 e 2 acima, entretanto $\|I\|=\sqrt{n}$. Mais informações sobre normas matriciais podem ser obtidas em [7]. 


\section{B.3}

Topologia

Definição B.11 (Bolas em $\mathbb{R}^{n}$ ) [2, 4]

Seja $x \in \mathbb{R}^{n}$ e $r>0$ então:

1. O conjunto $B_{r}(x):=\left\{y \in \mathbb{R}^{n}:\|x-y\|<r\right\}$ é denominado de Bola Aberta de raio r centrada em $x$.

2. O conjunto $\overline{B_{r}}(x):=\left\{y \in \mathbb{R}^{n}:\|x-y\| \leq r\right\}$ é denominado de Bola Fechada de raio $r$ centrada em $x$.

\section{Definição B.12 (Topologia em $\mathbb{R}^{n}$ ) [2, 4]}

Seja $G \subseteq \mathbb{R}^{n}$ então definimos:

1. $G$ é aberto se para todo ponto $x \in G$ existir $r>0$ tal que $B_{r}(x) \subset G$.

2. Gé fechado se $\mathbb{R}^{n} \backslash G$ for aberto.

3. $G$ é uma vizinhança de $x \in \mathbb{R}^{n}$ se $G$ for um aberto tal que $x \in G$.

4. $x \in \mathbb{R}^{n}$ é denominado um ponto de fronteira de $G$ se toda vizinhança de $x$ contém um ponto em $G$ e outro em $\mathbb{R}^{n} \backslash G$.

5. O conjunto dos pontos de fronteira de $G$ é denotado como $\partial G$.

\section{Teorema B.13 (Teoremas Gerais de Topologia no $\mathbb{R}^{n}$ ) [2, 4]}

1. $F \subset R^{n}$ é fechado se e somente se $\partial F \subseteq F$.

2. A interseção finita de conjuntos abertos é um conjunto aberto.

3. A união de qualquer coleção de conjuntos abertos é um conjunto aberto

4. A união finita de conjuntos fechados é um conjunto fechado.

5. A interseção de qualquer coleção de conjuntos fechados é um conjunto fechado.

\section{Teorema B.14 (Teorema de Heine-Borel) [2]}

$K \subset \mathbb{R}^{n}$ é compacto [2], se e somente se, $K$ é fechado e limitado.

\section{Proposição B.15 (Distância de um ponto a um conjunto) [5]}

Seja $A \subset \mathbb{R}^{n}$ e $f: \mathbb{R}^{n} \rightarrow \mathbb{R}$ tal que para todo $x \in \mathbb{R}^{n}$ :

$$
f(x):=\operatorname{dist}(x, A)=\inf _{y \in A}\|x-y\|
$$

então f é uniformemente contínua. 


\section{B.4 \\ Sequências e Séries}

Os conceitos e teoremas apresentados nesta seção sobre sequências e séries em $\mathbb{R}^{n}$ também se aplicam às sequências e séries de matrizes, utilizando normas matriciais $[7,8]$ ou considerando a bijeção entre as matrizes em $\mathbb{R}^{n \times n}$ e vetores em $\mathbb{R}^{n^{2}}$.

\section{Definição B.16 (Limite de Sequências) [4]}

Dizemos que $x \in \mathbb{R}^{n}$ é o limite da sequência $\left(x_{k}\right)_{k \in \mathbb{N}}$ ou simplesmente que $\left(x_{k}\right)_{k \in \mathbb{N}}$ converge para $x$, quando para todo $\varepsilon>0$ existe $N \in \mathbb{N}$ tal que sempre que $k>N$ tivermos $\left\|x_{k}-x\right\|<\varepsilon$.

Se existir este limite, escreve-se:

$$
\lim _{k \rightarrow \infty} x_{k}=x \quad, \quad \lim x_{k}=x \quad \text { e } \quad x_{k} \rightarrow x
$$

\section{Definição B.17 (Sequência de Cauchy) [2]}

Seja $\left(x_{k}\right)_{k \in \mathbb{N}}$ sequência em $\mathbb{R}^{n}$, dizemos que $\left(x_{k}\right)$ é uma sequência de Cauchy se para todo $\varepsilon>0$ existir $N \in \mathbb{N}$ tal que para todo $i, j \geq N$ tivermos $\left\|x_{i}-x_{j}\right\|<\varepsilon$.

\section{Teorema B.18 (Critério de Cauchy para Séries) [2]}

Uma série $\sum_{k=1}^{\infty} a_{k}$ em que $a_{k} \in \mathbb{R}^{n}$ para todo $k \in \mathbb{N}$ é convergente, se e somente se, dado $\varepsilon>0$ existir $N \in \mathbb{N}$ tal que para todo $i>j \geq N$ tivermos $\left\|\sum_{k=j}^{i} a_{k}\right\|<\varepsilon$.

\section{Definição B.19 (Séries Absolutamente Convergentes) [2]}

Seja $\left(x_{k}\right)_{k \in \mathbb{N}}$ sequência em $\mathbb{R}^{n}$, dizemos que a série $\sum x_{k}$ é absolutamente convergente se a série $\sum\left\|x_{k}\right\|$ é convergente.

\section{Teorema B.20 (Convergência Absoluta de Séries) [2]}

Seja $\left(x_{k}\right)_{k \in \mathbb{N}}$ sequência em $\mathbb{R}^{n}$, se a série $\sum x_{k}$ é absolutamente convergente então $\sum x_{k}$ é convergente.

Definição B.21 (Euler) Um exemplo conhecido de série convergente é o número de Euler:

$$
e^{x}=\sum_{k=0}^{\infty} \frac{x^{k}}{k !}
$$


Teorema B.22 (Teoremas de Sequências em $\mathbb{R}^{n}$ ) [2, 4]

Sejam $\left(x_{k}\right)_{k \in \mathbb{N}} e\left(y_{k}\right)_{k \in \mathbb{N}}$ sequências em $\mathbb{R}^{n}$ :

1. Toda sequência possui no máximo um único limite. [Unicidade]

2. $\left(x_{k}\right)_{k \in \mathbb{N}}$ é convergente, se e somente se, $\left(x_{k}\right)_{k \in \mathbb{N}}$ é de Cauchy (B.17).

3. Se $\left(x_{k}\right)_{k \in \mathbb{N}}$ é limitada então existe uma subsequência $\left(x_{k_{i}}\right)_{i \in \mathbb{N}}$ convergente [Teorema de Bolzano-Weirstrass].

4. Se $\left(x_{k}\right)_{k \in \mathbb{N}}$ é convergente então $\left(x_{k}\right)_{k \in \mathbb{N}}$ é limitada.

5. $\left(x_{k}\right)_{k \in \mathbb{N}}$ converge para $x$, se e somente se, toda subsequência $\left(x_{k_{i}}\right)_{i \in \mathbb{N}}$ também converge para $x$.

6. Se $x_{k} \rightarrow x$ e $y_{k} \rightarrow y$ então $\left(x_{k} \pm y_{k}\right) \rightarrow(x \pm y)$ e $\left(x_{k} . y_{k}\right) \rightarrow x . y$.

7. Seja $F \subseteq \mathbb{R}^{n}$ fechado. Se $x_{k} \in F$ para todo $k \in \mathbb{N}$ e $x_{k} \rightarrow x$ então $x \in F$.

8. Seja $K \subset \mathbb{R}^{n}$ compacto. Se $x_{k} \in K$ para todo $k \in \mathbb{N}$ então existe uma subsequência $\left(x_{k_{i}}\right)_{i \in \mathbb{N}}$ que converge para um ponto $x \in K$. [4]

\section{Teorema B.23 (Teoremas de Sequências em $\mathbb{R}$ ) [1, 3]}

Além de serem válidos todos os itens do Teorema B.22 para $n=1$, temos também que sejam $\left(x_{k}\right)_{k \in \mathbb{N}},\left(y_{k}\right)_{k \in \mathbb{N}},\left(z_{k}\right)_{k \in \mathbb{N}}$ sequências em $\mathbb{R}$ :

1. Se $\left(x_{k}\right)_{k \in \mathbb{N}}$ é monótona e limitada então $\left(x_{k}\right)_{k \in \mathbb{N}}$ é convergente.

2. Se $y_{k} \rightarrow y, x_{k} \rightarrow x, z_{k} \rightarrow z$ e $y_{k} \leq x_{k} \leq z_{k}$ para todo $k \in \mathbb{N}$ então $y \leq x \leq z$.

3. Se $y_{k} \rightarrow x, z_{k} \rightarrow x$ e $y_{k} \leq x_{k} \leq z_{k}$ para todo $k \in \mathbb{N}$ então $x_{k} \rightarrow x$. [Teorema do Sanduíche] 


\section{B.5}

\section{Limites e Continuidade}

Os conceitos e teoremas apresentados nesta seção também se aplicam às funções matriciais $f: D \subseteq \mathbb{R}^{n} \rightarrow \mathbb{R}^{m \times m}$, utilizando normas matriciais ou considerando a bijeção entre matrizes $\mathbb{R}^{m \times m}$ e vetores em $\mathbb{R}^{m^{2}} \cdot[7,8]$

\section{Definição B.24 (Limite de Funções) [4]}

Seja $f: D \subseteq \mathbb{R}^{n} \rightarrow \mathbb{R}^{m}, x_{0} \in \mathbb{R}^{n}, b \in \mathbb{R}^{m}$ dizemos que L é o limite de $f(x)$ quando $x$ tende para $x_{0}$ e escrevemos $\lim _{x \rightarrow x_{0}} f(x)=L$ quando para todo $\varepsilon>0$ existe $\delta>0$ tal que se $x \in D$ e $0<\left\|x-x_{0}\right\|<\delta$ implicar que $\|f(x)-L\|<\varepsilon$.

\section{Teorema B.25 (Operações com Limites de Funções) [4]}

Sejam $f, g: D \subseteq \mathbb{R}^{n} \rightarrow \mathbb{R}^{m}, \alpha: D \subseteq \mathbb{R}^{n} \rightarrow \mathbb{R}$ e $x_{0} \in D$.

Se existirem:

$$
\lim _{x \rightarrow x_{0}} f(x)=a \quad, \quad \lim _{x \rightarrow x_{0}} g(x)=b \quad \text { e } \quad \lim _{x \rightarrow x_{0}} \alpha(x)=\alpha_{0}
$$

então existem os limites e valem as seguintes igualdades:

$$
\begin{array}{ccc}
\lim _{x \rightarrow x_{0}}(f(x) \pm g(x))=a \pm b & , \quad \lim _{x \rightarrow x_{0}} \alpha(x) f(x)=\alpha_{0} a \\
\lim _{x \rightarrow x_{0}} f(x) . g(x)=a . b \quad e & \lim _{x \rightarrow x_{0}}\|f(x)\|=\|a\| .
\end{array}
$$

$E$, se $f(x) \leq g(x)$ (ou $f(x) \geq g(x)$ ) para todo $x \in D$ então $a \leq b$ (ou $a \geq b$ ). Ainda, se $\lim _{x \rightarrow x_{0}}\|f(x)\|=0$ então $\lim _{x \rightarrow x_{0}} f(x)=0 \in \mathbb{R}^{m}$.

\section{Definição B.26 (Continuidade de Funções) [4]}

Seja $f: D \subseteq \mathbb{R}^{n} \rightarrow \mathbb{R}^{m}$ dizemos que $f$ é contínua em $x_{0} \in D$ se para todo $\varepsilon>0$ existe $\delta>0$ tal que se $x \in D$ e $\|x-a\|<\delta$ implicar que $\left\|f(x)-f\left(x_{0}\right)\right\|<\varepsilon$ Se $f$ for contínua para todo $x \in X \subseteq D$ dizemos que $f$ é contínua em $X$ e denotamos $f \in C(X)$.

\section{Definição B.27 (Continuidade Uniforme de Funções) [4]}

Seja $f: D \subseteq \mathbb{R}^{n} \rightarrow \mathbb{R}^{m}$ dizemos que $f$ é uniformemente contínua em $D$ se para todo $\varepsilon>0$ existe $\delta>0$ tal que se $x, y \in D\|x-y\|<\delta$ então $\left\|f(x)-f\left(x_{0}\right)\right\|<\varepsilon$.

Teorema B.28 (Máximo, Mínimo e Preservação de Compactos) [2] Seja o compacto $K \subset \mathbb{R}^{n}$ e $f: K \rightarrow \mathbb{R}^{m}$ contínua em $K$ então $f(K)$ (imagem de f) é compacto e existem pontos $x$ e $y \in K$ tais que:

$$
\|f(x)\|=\sup _{z \in K}\|f(z)\|=\max _{z \in K}\|f(z)\| \quad e \quad\|f(y)\|=\inf _{z \in K}\|f(z)\|=\min _{z \in K}\|f(z)\| .
$$


Definição B.29 (Condição de Lipschitz) [2]

Seja $f: D \subseteq \mathbb{R}^{n} \rightarrow \mathbb{R}^{m}$. Se existe $L>0$ para todo $x$ e $y \in D$ tal que:

$$
\|f(x)-f(y)\| \leq L\|x-y\|
$$

dizemos que $f$ é Lipschitz em D. Se para todo $x \in D$ existir uma vizinhança de $x$ em que $f$ é Lipschitz nesta vizinhança então dizemos que $f$ é localmente Lipschitz em D.

Sejam $D_{1} \times D_{2}=D, t \in D_{1}$ (fixo) e $f(t, x)$ em que $f: D_{1} \times D_{2} \rightarrow \mathbb{R}^{m}$. Se existe $L>0$ para todo $x$ e $y \in D_{2}$ tal que:

$$
\|f(t, x)-f(t, y)\| \leq L\|x-y\|
$$

dizemos que $f$ é Lipschitz com respeito a $x$ em $D$. Se para todo $(t, x) \in D$ existir uma vizinhança de $(t, x)$ em que $f$ é Lipschitz com respeito a $x$ nesta vizinhança então dizemos que fé localmente Lipschitz com respeito a $x$ em $D$.

\section{Teorema B.30 (Continuidade de Funções) [4]}

Sejam $f, g: D \subseteq \mathbb{R}^{n} \rightarrow \mathbb{R}^{m}, X=f(D)$ (imagem de $f$ ) e $h: X \rightarrow \mathbb{R}^{p}$.

1. Se $f$ e g forem contínuas em $x_{0} \in D$ então $(f \pm g)$ e $(f . g)$ são contínuas em $x_{0}$.

2. Se $f$ é contínua em $x_{0}$ e $h$ é contínua no ponto $f\left(x_{0}\right)$ então a função composta $h_{o} f$ é contínua em $x_{0}$.

3. $f$ é contínua em $x_{0}$, se e somente se, para toda sequência de pontos $x_{k} \in D$ tal que $x_{k} \rightarrow x_{0}$ tem-se $\lim f\left(x_{k}\right)=f\left(x_{0}\right)$.

4. $f$ é contínua em $x_{0}$, se e somente se, suas funções coordenadas $f_{1}, f_{2}, \ldots, f_{n}: D \rightarrow \mathbb{R}$ forem contínuas em $x_{0}$.

5. Se D for compacto então $f$ é uniformemente contínua em D.

6. Se f for Lipschitz então $f$ é uniformemente contínua. 


\section{B.6}

\section{Diferenciabilidade e Integração}

Definição B.31 [4] Sejam $I \subseteq \mathbb{R}$ e $f: I \rightarrow \mathbb{R}^{m}$ então dizemos que $f$ é diferenciável em $t_{0} \in I$ se existir o limite:

$$
f^{\prime}\left(t_{0}\right)=\lim _{h \rightarrow 0} \frac{f\left(t_{0}+h\right)-f\left(t_{0}\right)}{h}
$$

em que $f^{\prime}\left(t_{0}\right)$ é a derivada de $f$ no ponto $t_{0}$. Se $f$ for diferenciável para todo $t_{0} \in D \subseteq I$ então dizemos que $f$ é diferenciável em $D$. Se $f$ for diferenciável em $D$ e $f^{\prime}$ for contínua em $D$ então dizemos que $f \in C^{1}(D)$.

Definição B.32 No caso das funções matriciais em que $I \subseteq \mathbb{R} e$ $A: I \rightarrow \mathbb{R}^{m \times m}$ dizemos que $A$ é diferenciável em $t_{0} \in I$ se existir o limite:

$$
A^{\prime}\left(t_{0}\right)=\lim _{h \rightarrow 0} \frac{A\left(t_{0}+h\right)-A\left(t_{0}\right)}{h}
$$

em que $A^{\prime}\left(t_{0}\right)$ é a derivada de $f$ no ponto $t_{0}$. Ainda, representando matrizes em $\mathbb{R}^{m \times m}$ como vetores em $\mathbb{R}^{m^{2}}$, podemos aplicar os teoremas apresentados nesta seção.

Definição B.33 [2, 4] Sejam $G \subseteq \mathbb{R}^{n}, f: G \rightarrow \mathbb{R}^{m}$ e $i=1, \ldots, n$.

Definimos a $i$-ésima derivada parcial de $f$ no ponto $x_{0} \in \mathbb{R}^{n}$, se existir, como:

$$
D_{i} f\left(x_{0}\right):=\frac{\partial f}{\partial x_{i}}\left(x_{0}\right)=\left(\frac{\partial f_{1}}{\partial x_{i}}\left(x_{0}\right), \ldots, \frac{\partial f_{m}}{\partial x_{i}}\left(x_{0}\right)\right)=\lim _{t \rightarrow 0} \frac{f\left(x_{0}+t e_{i}\right)-f\left(x_{0}\right)}{t}
$$

em que $e_{i}$ é o vetor canônico de $\mathbb{R}^{n}$, ou seja, $e_{i}=(0, \ldots, 1,0, \ldots, 0)$ sua $i$ ésima coordenada é igual a 1 e as demais são iguais a 0 (zero).

No caso particular em que $m=1$ definimos o vetor gradiente de $f$ como:

$$
\nabla f:=f^{\prime}=\left(\frac{\partial f}{\partial x_{1}}, \ldots, \frac{\partial f}{\partial x_{n}}\right) .
$$

Definição B.34 [2] Sejam $G \subseteq \mathbb{R}^{n}$ (aberto), $x_{0} \in G$ e $f: G \rightarrow \mathbb{R}^{m}$.

Dizemos que $f$ é diferenciável em $x_{0}$ se existir uma aplicação linear $L: \mathbb{R}^{n} \rightarrow \mathbb{R}^{m}$ em que para todo $\varepsilon>0$ existe $\delta>0$ tal que se $u \in \mathbb{R}^{n}$ e $\|u\| \leq \delta$ então:

$$
\left\|f\left(x_{0}+u\right)-f\left(x_{0}\right)-L . u\right\| \leq \varepsilon\|u\| .
$$

Neste caso, denominamos $L$ de derivada de $f$ no ponto $x_{0}$ e denotamos $D f\left(x_{0}\right):=L$. Se $f$ for diferenciável para todo $x_{0} \in D \subseteq G$ então dizemos que $f$ é diferenciável em $D$. 
Teorema B.35 [2] Sejam $G \subseteq \mathbb{R}^{n}$ e $f: G \rightarrow \mathbb{R}^{m}$.

Se $f$ for diferenciável em $x_{0} \in G$ então existem todas as derivadas parciais de $f$ em $x_{0}$ e temos que a derivada de $f$ em $x_{0}$ está associada a seguinte matriz $(m \times n)$ :

$$
f^{\prime}\left(x_{0}\right):=D f\left(x_{0}\right):=\left[\begin{array}{ccc}
D_{1} f_{1}\left(x_{0}\right) & \ldots & D_{n} f_{1}(a) \\
\ldots & \ldots & \ldots \\
D_{1} f_{m}\left(x_{0}\right) & \ldots & D_{n} f_{m}\left(x_{0}\right)
\end{array}\right] .
$$

Se $f$ for diferenciável em $D \subseteq G$ então $f \in C^{1}(D)$, se e somente se, suas derivadas parciais existirem e forem contínuas em $D$.

Teorema B.36 (Continuidade) [2, 4]

Se $f: G \subseteq \mathbb{R}^{n} \rightarrow \mathbb{R}^{m}$ é diferenciável em $x_{0} \in G$ então $f$ é contínua em $x_{0}$.

\section{Teorema B.37 (Teorema do Valor Médio) [2]}

Sejam $G \subseteq \mathbb{R}^{n}$ aberto, $f: G \rightarrow \mathbb{R}, a \in G, b \in G$ e $f$ diferenciável no segmento de reta que liga os pontos a e b então existe um ponto c neste segmento tal que:

$$
f(b)-f(a)=\nabla f(c) \cdot(b-a) .
$$

\section{Teorema B.38 (Regra da Cadeia) [2, 4]}

Sejam $f: A \subseteq \mathbb{R}^{n} \rightarrow \mathbb{R}^{m}, g: B \subseteq \mathbb{R}^{m} \rightarrow \mathbb{R}^{p}$ tais que $f$ é diferenciável em $c \in \mathbb{R}^{n}$ e g é diferenciável em $b=f(c)$ então a composta $h:=g_{o} f$ é diferenciável em c e sua derivada é o produto das matrizes $D g(b)$ e $D f(c)$ :

$$
h^{\prime}(c)=D h(c)=D g(b) D f(c) \quad\left(h^{\prime}(c) \in \mathbb{R}^{p \times n}\right) .
$$

A seguir, destacamos os seguintes casos particulares:

1. $p=1$ :

$$
\frac{\partial h}{\partial x_{i}}(c)=\sum_{k=1}^{m} \frac{\partial g}{\partial y_{k}}(b) \frac{\partial f_{k}}{\partial x_{i}}(c) \quad i=1 \ldots n \quad\left(h^{\prime}(c) \in \mathbb{R}^{n}\right)
$$

2. $n=m=p=1$ :

$$
h^{\prime}(c)=g^{\prime}(b) f^{\prime}(c) \quad\left(h^{\prime}(c) \in \mathbb{R}\right) .
$$


Teorema B.39 (Composição de funções) [2, 4]

Sejam $f, g: G \subseteq \mathbb{R}^{n} \rightarrow \mathbb{R}^{m}$ diferenciáveis em $x_{0} \in G$.

1. Então $(f \pm g)$ é diferenciável em $x_{0} e(f \pm g)^{\prime}\left(x_{0}\right)=f^{\prime}\left(x_{0}\right) \pm g^{\prime}\left(x_{0}\right)$.

2. Se $u \in \mathbb{R}^{n}$ então o produto escalar $h(x):=f(x) . g(x)$ é diferenciável em $x_{0}$ e $h^{\prime}\left(x_{0}\right) \cdot u=f^{\prime}\left(x_{0}\right) u \cdot g\left(x_{0}\right)+f\left(x_{0}\right) \cdot\left(g^{\prime}\left(x_{0}\right) u\right)$.

No caso particular $n=1$, temos $h^{\prime}\left(x_{0}\right)=f^{\prime}\left(x_{0}\right) \cdot g\left(x_{0}\right)+f\left(x_{0}\right) \cdot g^{\prime}\left(x_{0}\right)$.

Teorema B.40 (Teorema Fundamental do Cálculo) [3]

Sejam $f: I \subseteq \mathbb{R} \rightarrow \mathbb{R}$ contínua em $I$ e $F: I \rightarrow \mathbb{R}$ então as seguintes afirmações são equivalentes:

1. F é uma integral indefinida de $f$, ou seja, existe $x_{0} \in I$ tal que $F(x)=F\left(x_{0}\right)+\int_{x_{0}}^{x} f(t) d t$ para todo $t \in I$.

2. F é uma primitiva de $f$, ou seja, $F^{\prime}(x)=f(x)$ para todo $x \in I$. 


\section{B.7}

\section{Convergência Uniforme}

Os conceitos e teoremas apresentados nesta seção também se aplicam às funções matriciais $f: D \subseteq \mathbb{R}^{n} \rightarrow \mathbb{R}^{m \times m}$, utilizando normas matriciais ou considerando a bijeção entre matrizes $\mathbb{R}^{m \times m}$ e vetores em $\mathbb{R}^{m^{2}} \cdot[7,8]$

\section{Definição B.41 (Convergência Uniforme de Funções) [2]}

Uma sequência de funções $\left\{f_{k}\right\}_{k \in \mathbb{N}}$ em que $f_{k}: G \subseteq \mathbb{R}^{n} \rightarrow \mathbb{R}^{m}$ converge uniformemente em $D \subseteq G$ para uma função $f: D \rightarrow \mathbb{R}^{m}$ se para todo $\varepsilon>0$ existir $N \in \mathbb{N}$ tal que para todo $k \geq N$ tivermos:

$$
\sup _{x \in D}\left\|f_{k}(x)-f(x)\right\|<\varepsilon
$$

Teorema B.42 (Cauchy: Convergência Uniforme de Funções) [2]

Seja $\left\{f_{k}\right\}_{k \in \mathbb{N}}$ uma sequência de funções limitadas $f_{k}: D \subseteq \mathbb{R}^{n} \rightarrow \mathbb{R}^{m}$. Então existe uma função limitada $f: D \subseteq \mathbb{R}^{n} \rightarrow \mathbb{R}^{m}$ tal que $\left\{f_{k}\right\}_{k \in \mathbb{N}}$ converge uniformemente para $f$ em $D$, se e somente se, para todo $\varepsilon>0$ existir $N \in \mathbb{N}$ tal que para todo $i, j \geq N$ tivermos:

$$
\sup _{x \in D}\left\|f_{i}(x)-f_{j}(x)\right\|<\varepsilon
$$

\section{Definição B.43 (Convergência Uniforme de Séries) [2]}

Uma série de funções $\sum f_{k}$ com $f_{k}: G \subseteq \mathbb{R}^{n} \rightarrow \mathbb{R}^{m}$ converge uniformemente em $D \subseteq G$ para uma função $f: D \rightarrow \mathbb{R}^{m}$ se a sequência de funções $\left\{s_{k}\right\}_{k \in \mathbb{N}}$ com $s_{k}=\sum_{i=1}^{k} f_{i}$ convergir uniformemente em $D$.

\section{Proposição B.44 (Cauchy: Convergência Uniforme de Séries) [2]}

Seja $\left\{f_{k}\right\}_{k \in \mathbb{N}}$ uma sequência de funções $f_{k}: D \subseteq \mathbb{R}^{n} \rightarrow \mathbb{R}^{m}$. A série $\sum f_{k}$ converge uniformemente em $D$, se e somente se, para todo $\varepsilon>0$ existir $N \in \mathbb{N}$ tal que para todo $i \geq j \geq N$ tivermos:

$$
\sup _{x \in D}\left\|\sum_{k=j}^{i} f_{k}(x)\right\|<\varepsilon .
$$

\section{Teorema B.45 (Continuidade e Convergência Uniforme) [2]}

Seja $\left\{f_{k}\right\}_{k \in \mathbb{N}}$ uma sequência de funções contínuas $f_{k}: D \subseteq \mathbb{R}^{n} \rightarrow \mathbb{R}^{m}$ tal que $f_{k}$ converge uniformemente para $f$ em $D$ então $f$ é contínua em $D$. 
Corolário B.46 O Teorema B.45 também se aplica as séries de funções $\sum f_{k}$, considerando a sequência de funções $s_{k}$ tal que $s_{k}=\sum_{i=1}^{k} f_{i}$.

Teorema B.47 (Derivação e Convergência Uniforme) [1, 2, 3$]$

Sejam $I \subset \mathbb{R}$ um intervalo limitado $e\left\{f_{k}\right\}_{k \in \mathbb{N}}$ uma sequência de funções diferenciáveis em $I$ em que $f_{k}: I \rightarrow \mathbb{R}^{m}$.

Se existe algum $x_{0} \in I$ tal que a sequência $\left(f\left(x_{0}\right)_{k}\right)_{k \in \mathbb{N}}$ é convergente e a sequência de funções $\left\{f^{\prime}{ }_{k}\right\}_{k \in \mathbb{N}}$ converge uniformemente para $g$ em I então a sequência de funções $\left\{f_{k}\right\}_{k \in \mathbb{N}}$ converge uniformemente para uma função $f$ diferenciável em I tal que $f^{\prime}=g$, ou seja:

$$
\lim _{k \rightarrow \infty} f_{k}^{\prime}(x)=f^{\prime}(x)=\left(\lim _{k \rightarrow \infty} f_{k}(x)\right)^{\prime} \quad \forall x \in I .
$$

Corolário B.48 [1, 2, 3] O Teorema B.47 também se aplica as séries de funções $\sum f_{k}$, considerando a sequência de funções $s_{k}$ tal que $s_{k}=\sum_{i=1}^{k} f_{i}$.

Teorema B.49 (Integração e Convergência Uniforme) [1, 2, 3]

Sejam $I=[a, b] \subset \mathbb{R} e\left\{f_{k}\right\}_{k \in \mathbb{N}}$ uma sequência de funções integráveis em $I$ em que $f_{k}: I \rightarrow \mathbb{R}^{m}$ tal que $f_{k}$ converge uniformemente para $f$ em $I$ então $f$ é integrável em I e:

$$
\lim _{k \rightarrow \infty} \int_{a}^{b} f_{k}=\int_{a}^{b} f
$$




\section{B.8}

\section{Matrizes}

Definição B.50 (Função Multilinear) [6]

Seja $D: K^{n} \times K^{n} \times \cdots \times K^{n} \rightarrow K$ uma função de $n$ variáveis $A^{1}, \ldots A^{n}$, cada uma delas em $K^{n}$, onde $K$ é um campo. Dizemos que $D$ é multilinear se satisfizer as seguintes propriedades:

1. $D\left(A^{1}, \ldots, C+C^{\prime}, \ldots, A^{n}\right)=D\left(A^{1}, \ldots, C, \ldots, A^{n}\right)+D\left(A^{1}, \ldots, C^{\prime}, \ldots, A^{n}\right)$ para todo $C$ e $C^{\prime} \in K^{n}$.

2. $D\left(A^{1}, \ldots, \alpha C, \ldots, A^{n}\right)=\alpha D\left(A^{1}, \ldots, C, \ldots, A^{n}\right)$ para todo $C \in K^{n} e$ $\alpha \in K$.

Dizemos que D é alternante se, sempre que $A^{j}=A^{j+1}$ para algum $j$, tivermos:

$$
D\left(A^{1}, \ldots, A^{j}, A^{j+1}, \ldots, A^{n}\right)=0 .
$$

Teorema B.51 (Determinante) [6]

Existe uma única função multilinear alternante (Definição B.50) tal que $D(I)=1$. Denominamos esta função de Determinante, definida como:

$$
|A|:=\operatorname{det}(A)=D(A)=\sum_{j=1}^{n}(-1)^{i+j} a_{i j}\left|A_{i j}\right|=\sum_{i=1}^{n}(-1)^{i+j} a_{i j}\left|A_{i j}\right|
$$

em que fixamos $i \leq n$ (ou $j \leq n$ ), $\left(a_{i j}\right)$ é o ij-ésimo elemento da matriz $A$ $(n \times n)$ e $A_{i j}$ é a matriz $(n-1) \times(n-1)$ obtida retirando-se a i-ésima linha e j-ésima coluna de A.

A função determinante satisfaz também as seguintes propriedades:

1. Se duas colunas (ou linhas) forem trocadas então o determinante muda de sinal.

2. Se duas colunas (ou linhas) forem iguais então o determinante é nulo.

3. Se for adicionado um múltiplo de uma coluna (ou linha) em uma outra coluna (ou linha), o valor do determinante permanece inalterado.

4. Sejam $A$ e $B$ matrizes $(n \times n)$ então $D(A B)=D(A) \cdot D(B)$.

5. D é uma função contínua. 


\section{Teorema B.52 (Inversa de uma Matriz) [6]}

Seja $A=\left(a_{i j}\right)_{i, j=1}^{n}$ uma matriz $(n \times n)$ tal que $D(A) \neq 0$ em que $D$ é a função determinante (definida no Teorema B.51) então A é inversivel e sua inversa $B=\left(b_{i j}\right)_{i, j=1}^{n}$ é dada por:

$$
b_{i j}=\frac{D\left(A_{1}, \ldots, e_{j}, \ldots, A_{n}\right)}{D(A)}
$$

em que $e_{j}$ ocorre na i-ésima coordenada da função $D$ sendo o vetor canônico em $\mathbb{R}^{n}$ para todo $j \leq n$ e $A_{i}$ são as i-ésimas linhas (ou colunas) de $A$.

\section{Teorema B.53 (Matriz Não-Singular) [6]}

Seja A uma matriz $(n \times n)$, então as seguintes afirmações são equivalentes:

1. A é inversivel, ou seja, existe $A^{-1}$ tal que $A^{-1} A=A A^{-1}=I$ em que $I$ é a matriz identidade. Denominamos a matriz A como Não-Singular.

2. As colunas (e linhas) de A são linearmente independentes.

3. $D(A) \neq 0$, em que $D$ é a função determinante definida no Teorema B.51.

\section{Proposição B.54 (Equivalência entre Matrizes Complexas e Reais)}

Seja $z_{0} \in \mathbb{C}$ tal que $z_{0}:=a_{0}+b_{0} i$ com $a_{0}, b_{0} \in \mathbb{R}$ então $z_{0}$ pode ser representado por um vetor $\overrightarrow{z_{0}}:=\left(a_{0}, b_{0}\right) \in \mathbb{R}^{2}$ ou por uma matriz $Z_{0} \in \mathbb{R}^{2 \times 2}$ dada por:

$$
Z_{0}:=\left[\begin{array}{cc}
a_{0} & -b_{0} \\
b_{0} & a_{0}
\end{array}\right] .
$$

Assim, seja $Z \in \mathbb{C}^{n \times n}$ definida como $Z:=\left(z_{k j}\right)_{k, j=1}^{n}$ tal que para todo $k, j \leq n$ temos $z_{k j}=a_{k j}+i b_{k j}$ com $a_{k j}, b_{k j} \in \mathbb{R}$ então podemos representar $Z$ como uma matriz $Z_{\mathbb{R}} \in \mathbb{R}^{2 n \times 2 n}$ tal que:

$$
Z_{\mathbb{R}}:=\left[\begin{array}{ccc}
Z_{11} & \ldots & Z_{12} \\
\vdots & \ddots & \vdots \\
Z_{n 1} & \ldots & Z_{n n}
\end{array}\right] \quad \text { em que } \quad Z_{k j}:=\left[\begin{array}{cc}
a_{k j} & -b_{k j} \\
b_{k j} & a_{k j}
\end{array}\right] .
$$

Seja $R_{n}$ o conjunto de todas as matrizes reais $Z_{\mathbb{R}}$ (na forma acima), então $R_{n}$ é um subespaço vetorial real de dimensão $2 n^{2}$ e temos que existe bijeção entre o espaço vetorial das matrizes complexas $\mathbb{C}^{n \times n}$ e $R_{n}$, ou seja:

- para toda matriz complexa $Z \in \mathbb{C}^{n \times n}$ existe uma única matriz real $Z_{\mathbb{R}} \in R_{n}$. - para toda matriz real $Z_{\mathbb{R}} \in R_{n}$ existe uma única matriz complexa $Z \in \mathbb{C}^{n \times n}$. Assim, dizemos que $Z$ e $Z_{\mathbb{R}}$ são equivalentes.

Como consequência destas definições e das propriedades multiplicativas e aditivas das matrizes, temos que sejam $A$ e $B \in \mathbb{C}^{n \times n}$ então $\left(\alpha A^{k}+\beta B^{m}\right)$ é equivalente a $\left(\alpha A_{\mathbb{R}}^{k}+\beta B_{\mathbb{R}}^{m}\right)$ para todo $\alpha, \beta \in \mathbb{R}$ e $k, m \in \mathbb{N}$. Logo, a exponencial matricial $e^{Z}$ (Definição 3.14) também é equivalente a $e^{Z_{\mathbb{R}}}$. 


\section{B.9}

\section{Aplicações Lineares}

Definição B.55 (Aplicação Linear) [6]

Sejam $V$ e $W$ espaços vetoriais sobre um campo $K$, denominamos de Aplicação Linear $F: V \rightarrow W$ que satisfaz as seguintes propriedades:

1. $F(u+v)=F(u)+F(v)$ para todo elemento $u, v \in V e$;

2. $F(\alpha v)=\alpha F(v)$ para todo $\alpha \in K$ e $v \in V$.

Dizemos que F é:

- injetiva, se para todo $u, v \in V$ tais que $u \neq v$ tivermos $F(u) \neq F(v)$.

- sobrejetiva, se para todo $w \in W$ existir $v \in V$ tal que $F(v)=w$.

- bijetiva, se for injetiva e sobrejetiva.

- inversível, se existir uma aplicação linear $F^{-1}: W \rightarrow V$ tal que:

$$
F^{-1}(F(v))=v \text { e } F\left(F^{-1}(w)\right)=w \text { para todo } v \in V \text { e } w \in W .
$$

\section{Teorema B.56 (Isomorfismo) [6]}

Seja $F$ aplicação linear tal que $F$ é bijetiva então $F$ é inversível e sua inversa também é uma aplicação linear bijetiva. Neste caso, dizemos que F é um isomorfismo linear.

\section{Proposição B.57 (Núcleo e Aplicações Injetivas) [6]}

Seja $F$ aplicação linear, denominamos de Núcleo de $F$ o conjunto dos elementos $v \in V$ tal que $F(v)=0$, denotamos este conjunto como Ker $F$ e temos:

Ker $F=\{0\}$, se e somente se, $F$ for injetiva.

\section{Proposição B.58 (Imagem e Aplicações Sobrejetivas) [6]}

Seja $F$ aplicação linear, denominamos de Imagem de $F$ o conjunto dos elementos $w \in W$ em que existe $v \in V$ tal que $F(v)=w$, denotamos este conjunto como $\operatorname{Im} F$ e temos que $\operatorname{Im} F$ é subespaço de $W$. Temos também:

$$
\operatorname{Im} F=W \text {, se e somente se, } F \text { é sobrejetiva. }
$$

\section{Teorema B.59 (Dimensões do Núcleo e Imagem) [6]}

Seja F aplicação linear então:

$$
\operatorname{dim} V=\operatorname{dim} \operatorname{Ker} F+\operatorname{dim} \operatorname{Im} F .
$$




\section{B.10}

\section{Autovalores e a Forma Normal de Jordan}

Consideremos $A$ uma matriz (real ou complexa) $(n \times n)$.

Definição B.60 (Autovalor e Autovetor) [7, 8]

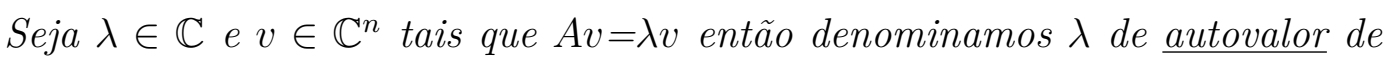
$A$ e $v$ seu respectivo autovetor.

\section{Definição B.61 (Polinômio Característico) [7, 8]}

Denominamos de polinômio característico de A:

$$
p(\lambda)=|A-\lambda I|=\left(\lambda-\lambda_{1}\right)^{m_{1}} \ldots\left(\lambda-\lambda_{l}\right)^{m_{p}}
$$

em que $\lambda_{1} \ldots \lambda_{p}$ são as raízes deste polinômio e $m_{1} \ldots m_{p}$ suas respectivas multiplicidades algébricas tal que $\sum_{i=1}^{p} m_{i}=n$ é o grau deste polinômio.

Teorema B.62 [7, 8] $\lambda$ é um autovalor de A, se e somente se, $\lambda$ for uma raiz do polinômio característico de A, ou seja:

$$
p(\lambda)=|A-\lambda I|=0 .
$$

\section{Teorema B.63 (Forma Normal de Jordan) ["7, 8 ]}

Existe uma matriz $S$ inversível que transforma uma matriz $A(n \times n)$ em uma matriz $J(n \times n)$ tal que $J=S^{-1} A S$ em que

$$
J:=\left[\begin{array}{ccc}
J_{1} & & 0 \\
& \ddots & \\
0 & & J_{k}
\end{array}\right](n \times n) \quad J_{j}:=\left[\begin{array}{cccc}
\lambda_{i(j)} & 1 & & 0 \\
& \ddots & \ddots & \\
& & \lambda_{i(j)} & 1 \\
0 & & & \lambda_{i(j)}
\end{array}\right]\left(n_{j} \times n_{j}\right)
$$

Com $j=1 \ldots k, i(j)=1 \ldots p$ e $\lambda_{i(j)}$ são as raízes do polinômio característico (Definição B.61), ou seja, são os autovalores de A (por B.62).

Denominamos cada bloco $J_{j}$ de bloco de Jordan de $A$ e a matriz $J$ de Forma Normal de Jordan de A. Note que, um mesmo autovalor $\lambda_{i}$ pode pertencer a mais de um bloco de Jordan.

Assim temos que $J=\Lambda+N$ em que $\Lambda$ é a matriz diagonal contendo os autovalores de $A$ e $N$ é a matriz dada por:

$$
N:=\left[\begin{array}{cccc}
0 & \delta_{1} & & 0 \\
& \ddots & \ddots & \\
& & \ddots & \delta_{n-1} \\
0 & & & 0
\end{array}\right] \text { em que } \delta_{i} \in\{0,1\} \text { para todo } i=1 \ldots(n-1) .
$$

